\title{
UNIFORM BOUNDS FOR STRONGLY COMPETING SYSTEMS: THE OPTIMAL LIPSCHITZ CASE
}

\author{
Nicola Soave \\ Justus Liebig Universität Giessen \\ Mathematisches Institut \\ Arndtstrasse 2, 35392, Giessen, Germany \\ email: nicola.soave@math.uni-giessen.de; nicola.soave@gmail.com
}

Alessandro Zilio

Centre d'analyse et de mathématique sociales

École des Hautes Études en Sciences Sociales

190-198 Avenue de France, 75244, Paris CEDEX 13

email: alessandro.zilio@polimi.it

\begin{abstract}
For a class of systems of semi-linear elliptic equations, including

$$
-\Delta u_{i}=f_{i}\left(x, u_{i}\right)-\beta u_{i} \sum_{j \neq i} a_{i j} u_{j}^{p}, \quad i=1, \ldots, k,
$$

for $p=2$ (variational-type interaction) or $p=1$ (symmetric-type interaction), we prove that uniform $L^{\infty}$ boundedness of the solutions implies uniform boundedness of their Lipschitz norm as $\beta \rightarrow+\infty$, that is, in the limit of strong competition. This extends known quasi-optimal regularity results and covers the optimal case for this class of problems. The proofs rest on monotonicity formulae of AltCaffarelli-Friedman and Almgren type in the variational setting, and on the Caffarelli-Jerison-Kenig almost monotonicity formula in the symmetric one.
\end{abstract}

\section{INTRODUCTION AND MAIN RESULTS}

In this paper we are concerned with the optimal uniform regularity of families of solutions to strongly competing systems either of Gross-Pitaevskii type:

$$
\begin{cases}-\Delta u_{i, \beta}+\lambda_{i, \beta} u_{i, \beta}=\omega_{i} u_{i, \beta}^{3}-\beta u_{i, \beta} \sum_{j \neq i} a_{i j} u_{j, \beta}^{2} & \text { in } \Omega \\ u_{i, \beta}>0 & \text { in } \Omega,\end{cases}
$$

or of Lotka-Volterra type:

$$
\begin{cases}-\Delta u_{i, \beta}+\lambda_{i} u_{i, \beta}=\omega_{i} u_{i, \beta}^{2}-\beta u_{i, \beta} \sum_{j \neq i} u_{j, \beta} & \text { in } \Omega \\ u_{i, \beta}>0 & \text { in } \Omega,\end{cases}
$$

with $i=1, \ldots, k$. In both cases, $\Omega \subset \mathbb{R}^{N}$ is a domain neither necessarily bounded, nor smooth, $\omega_{i} \in \mathbb{R}$, and $\beta$ is a positive parameter which has to be thought as tending to $+\infty$. In the previous setting, our main results read as follows.

Theorem 1.1. In dimension $N \leq 4$, let us assume that $a_{i j}=a_{j i}$ and $\left(\lambda_{i, \beta}\right)$ is a bounded sequence. Let $\left\{\mathbf{u}_{\beta}\right\}$ be a family of solutions of (1.1) uniformly bounded in $L^{\infty}(\Omega)$. Then for every compact set $\Omega^{\prime} \subset \subset \Omega$ there exists $M>0$ independent of $\beta$ such that

$$
\left\|\mathbf{u}_{\beta}\right\|_{\operatorname{Lip}\left(\Omega^{\prime}\right)}:=\left\|\mathbf{u}_{\beta}\right\|_{L^{\infty}\left(\Omega^{\prime}\right)}+\left\|\nabla \mathbf{u}_{\beta}\right\|_{L^{\infty}\left(\Omega^{\prime}\right)} \leq M .
$$

2010 Mathematics Subject Classification. Primary: 35B65; secondary: 35B25, 35J47, 35R35, 81Q05, 92D25.

Key words and phrases. optimal regularity theory, singular perturbation, systems of elliptic equations, monotonicity formulae. 
Theorem 1.2. In any dimension $N \geq 1$, let us assume that $\lambda_{i} \in \mathbb{R}$. Let $\left\{\mathbf{u}_{\beta}\right\}$ be a family of solutions of (1.2 uniformly bounded in $L^{\infty}(\Omega)$. Then for every compact set $\Omega^{\prime} \subset \subset \Omega$ there exists $M>0$ independent of $\beta$ such that

$$
\left\|\mathbf{u}_{\beta}\right\|_{\operatorname{Lip}\left(\Omega^{\prime}\right)}:=\left\|\mathbf{u}_{\beta}\right\|_{L^{\infty}\left(\Omega^{\prime}\right)}+\left\|\nabla \mathbf{u}_{\beta}\right\|_{L^{\infty}\left(\Omega^{\prime}\right)} \leq M .
$$

Here and in the rest of the paper we adopt the vector notation $\mathbf{u}=\left(u_{1}, \ldots, u_{k}\right)$.

1.1. Introduction to the problem. The study of the asymptotic behaviour of singularly perturbed equations and systems of elliptic type is a very broad and active subject of research. In recent years, a lot of interest has been given to systems of equations of competing densities, coming from chemical, biological, physical or purely mathematical applications. Typical examples of such systems can fit under the comprehensive model

$$
-\Delta u_{i}=f_{i}\left(x, u_{i}\right)-\beta g_{i}\left(u_{1}, \ldots, u_{k}\right) \quad \text { in } \Omega \subset \mathbb{R}^{N},
$$

where the functions $g_{i}$, modelling the interaction between the densities, can assume different shapes according to the underlying phenomena.

(I) For models coming from the physics, typically related to the Gross-Pitaevskii equations (see e.g. [22, 23, 26, 34]), the coupling between the different densities takes a variational form, as in

$$
g_{i}\left(u_{1}, \ldots, u_{k}\right)=u_{i} \sum_{j \neq i} a_{i j} u_{j}^{2} .
$$

Here the matrix $a_{i j}$ is assumed symmetric. This interaction is variational since one can easily see that the functions $g_{1}, \ldots, g_{k}$ are nothing but the partial derivatives of $G\left(u_{1}, \ldots, u_{k}\right)=$ $\sum_{i, j \neq i} a_{i j} u_{i}^{2} u_{j}^{2}$. These models are also of importance in other mathematical problems, such as the approximation of optimal partition problems and of harmonic maps to singular manifolds [7, 25].

(II) In biological or chemical application as in [20, 27], the interaction term is, in general, more symmetric, as it is derived from some probabilistic reasonings. In these situations one has, for instance,

$$
g_{i}\left(u_{1}, \ldots, u_{k}\right)=u_{i} \sum_{j \neq i} u_{j}
$$

Note that the lack of a variational structure is compensated by the symmetry of the competition. Great efforts have been directed to the description of a precise asymptotic of the solutions of the previous systems when the competition parameter $\beta$ diverges; with this we mean that the main goals have been:

(a) to develop a common regularity theory for the solutions of the system, which is independent of the strength of the competition $\beta>0$;

(b) to investigate under which assumptions one can guarantee convergence of the solutions to some limiting profile;

(c) to study the regularity of the class of limiting profiles, both in terms of the densities and in terms of the emerging free boundary problem;

(d) to give qualitative properties and precise estimates of such convergence.

This paper is mainly devoted to the improvement of the known results concerning the first point, since this serves as foundation for the subsequent ones. Before presenting our contribution, we give a brief review of the existing literature; this serves also as a motivation for our work.

1.2. Uniform bounds in Hölder spaces. The limiting behaviour of minimal solutions to variational systems of type (1.1) when $\beta \rightarrow+\infty$ has firstly been studied in [12, 13] by Conti, Terracini and Verzini in the so-called focusing case $\omega_{i}>0$ : it has been shown that any sequence of minimizers of the energy functional associated to 1.1 is convergent in $H^{1}(\Omega)$, as $\beta \rightarrow+\infty$, to a limiting profile $\mathbf{u}_{\infty}$ whose components have disjoint support, that is $u_{i, \infty} u_{j, \infty} \equiv 0$ a. e. in $\Omega$ for every $i \neq j$. This phenomenon, called phase separation or segregation, reflects the competitive nature of the considered type of interaction, and has been analysed also in the de-focusing case $\omega_{i}<0$ in [10] by Chang et. al. Afterwards, a breakthrough in the comprehension of the regularity issues of the phase separation have been achieved in [7, where for the first time Caffarelli and Lin have shown the $\mathcal{C}^{0, \alpha}$-uniform regularity for families of 
minimizers. As far as the excited states are concerned, probably the most relevant result available in the literature is the following.

Theorem (Noris, Tavares, Terracini, Verzini, 21]). In dimension $N \leq 3$, let us assume that $a_{i j}=a_{j i}$ and that $\left(\lambda_{i, \beta}\right)$ is a bounded sequence. Let $\left\{\mathbf{u}_{\beta}\right\} \subset H_{0}^{1}(\Omega)$ be a family of solutions of (1.1) uniformly bounded in $L^{\infty}(\Omega)$. Then for every $0<\alpha<1$ there exists $M>0$ independent of $\beta$ such that

$$
\left\|\mathbf{u}_{\beta}\right\|_{\mathcal{C}^{0, \alpha}(\bar{\Omega})} \leq M
$$

Up to a subsequence $\mathbf{u}_{\beta} \rightarrow \mathbf{u}_{\infty}$ in $\mathcal{C}^{0, \alpha}(\bar{\Omega})$ and in $H^{1}(\Omega)$, and $\mathbf{u}_{\infty}$ is a segregated configuration, that is $u_{i, \infty} u_{j, \infty} \equiv 0$ in $\Omega$ for every $i \neq j$.

Such a result extends and improves previous ones obtained by Wei and Weth in [38, where under the same assumptions the equi-continuity of $\left\{\mathbf{u}_{\beta}\right\}$ was proved in dimension $N=2$. We mention that in 38 . a wider class of systems is considered, including both (1.1) and (1.2).

Also in the symmetric setting phase separation phenomena arise in the limit $\beta \rightarrow+\infty$.

Theorem (Conti, Terracini, Verzini, [14). In dimension $N \geq 1$, let us assume that $\left(\lambda_{i, \beta}\right)$ is a bounded sequence. Let $\left\{\mathbf{u}_{\beta}\right\} \subset H^{1}(\Omega)$ be a family of solutions of $[1.2$ subjected to the boundary conditions

$$
u_{i}=\varphi_{i} \quad \text { on } \partial \Omega \text {, }
$$

where $\varphi_{i}$ are positive $\operatorname{Lip}(\partial \Omega)$-functions having disjoint supports. Then for every $0<\alpha<1$ there exists $M>0$ independent of $\beta$ such that

$$
\left\|\mathbf{u}_{\beta}\right\|_{\mathcal{C}^{0, \alpha}(\bar{\Omega})} \leq M
$$

Up to a subsequence $\mathbf{u}_{\beta} \rightarrow \mathbf{u}_{\infty}$ in $\mathcal{C}^{0, \alpha}(\bar{\Omega})$ and in $H^{1}(\Omega)$, and $\mathbf{u}_{\infty}$ is a segregated configuration.

We point out that $\left\{\mathbf{u}_{\beta}\right\}$ is uniformly bounded in $L^{\infty}(\Omega)$ as a consequence of the maximum principle. We also remark that the results in [14] are actually more general, in the sense that the reaction term $\omega_{i} u_{i}^{2}-\lambda_{i} u_{i}$ can be replaced by a general reaction term of type $f_{i}\left(x, u_{i}\right)$ (independent of $\beta$ ).

All the aforementioned results are global, in the sense that the solutions are assumed to be defined on smooth bounded domains of $\mathbb{R}^{N}$ and to satisfy suitable boundary conditions, and consequently the uniform estimates which are proved hold in the whole $\bar{\Omega}$.

Concerning the regularity of the limit configurations and of their free-boundary, we mainly refer to [7] for the variational setting, to [6] for the symmetric one, and in particular to [31, which provides a unified approach investigating the regularity of a wide class of segregated vector valued functions, including limiting configurations of both the classes of systems. Altogether, the main result which we want to recall in this setting can be stated as follows.

Theorem (Caffarelli et al. [6], Caffarelli and Lin 17, Tavares and Terracini [31). Under the assumptions of the previous theorems, let $\mathbf{u}_{\beta} \rightarrow \mathbf{u}_{\infty}$ in $\mathcal{C}^{0, \alpha}(\bar{\Omega})$ and in $H^{1}(\Omega)$ as $\beta \rightarrow+\infty$. Then $\mathbf{u}_{\infty}$ is Lipschitz continuous in $\Omega$.

As the limiting profile are not $\mathcal{C}^{1}$, the uniform Lipschitz regularity is optimal for this class of problems.

1.3. Uniform bounds in Lipschitz spaces. As a matter of fact, especially in the variational setting, it is still an open question whether one can deduce uniform bounds in the Lipschitz norm. The aim of this paper is to show that this is the case, in a rather general framework. Some results concerning uniform Lipschitz boundedness are already known in the literature, but in some specific cases.

In [2, Lemma 2.4] Berestycki, Lin, Wei and Zhao deal with the variational system $q=2$ in dimension $N=1$, proving the following. Let $\left\{\left(u_{\beta}, v_{\beta}\right)\right\} \in H_{0}^{1}([0,1])$ be solutions of

$$
\begin{cases}-u_{\beta}^{\prime \prime}+\lambda_{1, \beta} u_{\beta}=\omega_{1} u_{\beta}^{3}-\beta u_{\beta} v_{\beta}^{2} & \text { in }[0,1] \\ -v_{\beta}^{\prime \prime}+\lambda_{2, \beta} v_{\beta}=\omega_{2} v_{\beta}^{3}-\beta v_{\beta} u_{\beta}^{2} & \text { in }[0,1]\end{cases}
$$

with uniformly bounded coefficients $\left(\lambda_{i, \beta}\right)$. If $0 \leq u_{\beta}, v_{\beta} \leq C$, then $u_{\beta}$ and $v_{\beta}$ are uniformly bounded in the Lipschitz norm. The proof of such result heavily rests on the ODE aspects of the one dimensional Hamiltonian system. 
In 14, Theorem 3] Conti, Terracini and Verzini deal with the symmetric competition $q=1$. In the case of two components without reaction terms, they proved that if $\left\{\left(u_{\beta}, v_{\beta}\right)\right\} \in H^{1}(\Omega)$ are non-negative solutions of

$$
\begin{cases}\Delta u_{\beta}=\beta u_{\beta} v_{\beta} & \text { in } \Omega \\ \Delta v_{\beta}=\gamma \beta u_{\beta} v_{\beta} & \text { in } \Omega \\ u_{\beta}=\varphi, v_{\beta}=\psi & \text { in } \partial \Omega\end{cases}
$$

with $\gamma>0$ and traces $\varphi, \psi \in \operatorname{Lip}(\partial \Omega)$, then $\left\{\left(u_{\beta}, v_{\beta}\right)\right\}$ is uniformly bounded in the Lipschitz norm. With a different method, the result has been generalized to systems with an arbitrary number of components (possibly with suitable reaction terms) in [37. We refer the interested reader also to the paper [8], where it is possible to find some extensions of the previous result (involving different kinds of systems, but always restricted to the case of two components).

We emphasize that the existence of uniform Lipschitz bounds is relevant not only for a pure mathematical flavour. As already observed, it is necessary to obtain rigorous qualitative description of phase separation phenomena. This is clearly the case of [2, where the authors derived a precise decay rate for solutions of 1.3 on the interface $\left\{u_{\beta}=v_{\beta}\right\}$ in dimension $N=1$, strongly using the uniform Lipschitz boundedness of the solutions themselves (the Hölder bounds would not be sufficient for this purpose).

Our aim is twofold: we shall extend the optimal regularity to general cases and, in the mean time, we shall prove local versions of the regularity estimates, avoiding any assumptions on the boundary behaviour of the solutions. This is in the spirit of the classical elliptic regularity theory, and turns out to be particularly useful in blow-up analysis, when one has to deal with sequences of functions defined on expanding domains and hence the global estimates would not be applicable. We mention that a first step in this second direction can be found in [36, Theorem 2.6], where the author proves that the main results in 21] hold also in a local setting. We refer to [3, 17, 29, 30, 36, for several applications which rest upon the local nature of such statement. We refer also to the forthcoming paper 28 for further extensions, see Remark 2.4

Finally, we mention that uniform regularity issues have been considered for fully non-linear equations in [24, for non-local operators in [32, 33, 35, and in a parabolic setting in [16].

1.4. Main results. Concerning the optimal regularity problem, our main results, stated in the greatest possible generality, are the following.

Theorem 1.3 (Case (I)). Let $p \geq 1$ and let $\Omega \subset \mathbb{R}^{N}$ be neither necessarily bounded, nor necessarily smooth, where $N \leq 2(1+1 / p)$ is a positive integer. Let $\left\{\mathbf{u}_{\beta}\right\}$ be a family of weak solutions to

$$
\begin{cases}-\Delta u_{i, \beta}=f_{i, \beta}\left(x, u_{i, \beta}\right)-\beta u_{i, \beta}^{p} \sum_{j \neq i} a_{i j} u_{j, \beta}^{p+1} & \text { in } \Omega \\ u_{i, \beta}>0 & \text { in } \Omega,\end{cases}
$$

with $a_{i j}=a_{j i}$, uniformly bounded in $L^{\infty}(\Omega):\left\|\mathbf{u}_{\beta}\right\|_{L^{\infty}(\Omega)} \leq m$ for some $m>0$. Let us assume that $\mathbf{f}_{\beta} \in \mathcal{C}(\Omega \times \mathbb{R})$ are such that

$$
\max _{s \in[0, m]} \sup _{x \in \Omega}\left|\frac{f_{i, \beta}(x, s)}{s}\right| \leq d
$$

for some $d>0$; moreover for any sequence $\beta_{n} \rightarrow+\infty$ there exist a subsequence (still denoted $\beta_{n}$ ) and a function $\mathbf{f} \in \mathcal{C}^{1}(\Omega \times \mathbb{R})$ such that $\mathbf{f}_{\beta_{n}} \rightarrow \mathbf{f}$ in $\mathcal{C}_{\text {loc }}(\Omega \times \mathbb{R})$. Then for any $\Omega^{\prime} \subset \subset \Omega$ there exists $M>0$ such that

$$
\left\|\mathbf{u}_{\beta}\right\|_{\operatorname{Lip}\left(\bar{\Omega}^{\prime}\right)}=\left\|\mathbf{u}_{\beta}\right\|_{L^{\infty}\left(\bar{\Omega}^{\prime}\right)}+\left\|\nabla \mathbf{u}_{\beta}\right\|_{L^{\infty}\left(\bar{\Omega}^{\prime}\right)} \leq M .
$$

As a second result we establish the uniform Lipschitz boundedness of solutions of symmetric systems with an arbitrary number of components and general reaction terms, thus extending the results of [14, 37.

Theorem 1.4 (Case (II)). In any dimension $N \geq 1$, let $\Omega \subset \mathbb{R}^{N}$ be neither necessarily bounded, nor necessarily smooth. Let $p_{i} \geq 1$ for $i=1, \ldots, k$, and let $\left\{\mathbf{u}_{\beta}\right\}$ be a family of weak solutions to

$$
\begin{cases}-\Delta u_{i, \beta}=f_{i, \beta}\left(x, u_{i, \beta}\right)-\beta u_{i, \beta}^{p_{i}} \sum_{j \neq i} u_{j, \beta}^{p_{j}} & \text { in } \Omega \\ u_{i, \beta}>0 & \text { in } \Omega,\end{cases}
$$


uniformly bounded in $L^{\infty}(\Omega)$. Let us assume that $\mathbf{f}_{\beta}$ maps bounded sets in bounded sets, uniformly in $\beta$. Then, for any $\Omega^{\prime} \subset \subset \Omega$ there exists $M>0$ such that

$$
\left\|\mathbf{u}_{\beta}\right\|_{\operatorname{Lip}\left(\bar{\Omega}^{\prime}\right)}=\left\|\mathbf{u}_{\beta}\right\|_{L^{\infty}\left(\bar{\Omega}^{\prime}\right)}+\left\|\nabla \mathbf{u}_{\beta}\right\|_{L^{\infty}\left(\bar{\Omega}^{\prime}\right)} \leq M
$$

Theorems 1.1 and 1.2 follow straightforwardly.

Some remarks are in order.

Remarks. 1) In the variational case this is the first occurrence in the literature of semi-linear terms depending on the independent variable $x$ (in the symmetric setting, such a dependence has been already considered in [14]). This enables us actually to give an equivalent, but apparently more general, formulation of the assumptions allowing the reaction terms $f_{i, \beta}$ to depend upon $u_{1}, \ldots, u_{k}$ and, if needed, also on $\nabla u_{1}, \ldots, \nabla u_{k}$ in a uniformly bounded way. Above all, we stress this in order to point out that the locution variational and symmetric have to be referred to the type of interaction and not to the system per se.

2) The restriction on the dimension in the variational case (I) is mainly a technical assumption, related to the subcriticality of the potentials $u_{i}^{p+1} u_{j}^{p+1}$ associated to the interaction terms $u_{i}^{p} u_{j}^{p+1}$. We point out that it can be easily dropped if one requires, for instance, that the semi-linear terms satisfy the additional assumption

$$
f_{i, \beta}(x, s) \leq 0 \quad \text { for } s \in[0, m], i=1, \ldots, k .
$$

In [7] Caffarelli and Lin considered the variational system

$$
-\Delta u_{i}=-\beta u_{i} \sum_{j \neq i} u_{j}^{2}
$$

(without any internal reaction term, $f_{i, \beta} \equiv 0$ ). In their setting, they proved that minimal solutions to such system are uniformly bounded in $\mathcal{C}^{0, \alpha}(\bar{\Omega})$ for any $\alpha \in(0,1)$. The proof of Theorem 1.3 can be slightly modified as indicated in the forthcoming Remark 3.4 to obtain the following result, holding in any dimension.

Theorem 1.5. In dimension $N \geq 1$, let $\left\{\mathbf{u}_{\beta}\right\} \in H^{1}(\Omega)$ be a family of positive solutions of (1.7), uniformly bounded in $L^{\infty}(\Omega)$. Then for any $\Omega^{\prime} \subset \subset \Omega$ there exists $M>0$ such that

$$
\left\|\mathbf{u}_{\beta}\right\|_{\operatorname{Lip}\left(\bar{\Omega}^{\prime}\right)} \leq M
$$

3) Concerning the assumptions on the reaction terms in Theorem 1.3, we firstly stress that the precompactness assumption on $\left\{\mathbf{f}_{\beta}\right\}$, although technical at a first glance, is natural and is shared by all the known results in the literature. Indeed, when considering the particular system (1.1), it is simply given by the requirement that the sequence $\left(\lambda_{i, \beta}\right)$ is bounded. Regarding $(1.5)$, we point out that so far limiting configurations of systems of type (1.4) have been proved to be Lipschitz continuous in [21, 31] using such condition. Without it, the Lipschitz regularity of the limiting profiles is still an open problem. In this perspective, we emphasize that Theorem 1.4 not making use of (1.5), establishes the Lipschitz regularity of all limiting profiles of system (1.6) for a wider class of reaction terms with respect to those considered in the literature 31 .

4) In the symmetric setting, the possibility of considering different exponents $p_{i}$ can be used to obtain uniform bounds in more general models. Indeed, with the change of variable $v_{i}:=u_{i}^{p_{i}}$, equation 1.6 reads as

$$
-\Delta v_{i}^{1 / p_{i}}=f_{i}\left(x, v_{1}, \ldots, v_{k}\right)-\beta v_{i} \sum_{j \neq i} v_{j} \quad \text { in } \Omega
$$

that is, the Lotka-Volterra system for the fast-diffusion equation.

5) Concerning uniform regularity up to the boundary, we believe that all our results can be extended with some efforts to deal with systems of equations with elliptic operators with variable coefficients. Since we will make use of several monotonicity formulae, this would be very technical and not always easy; the reader can easily understand what we mean by looking at the contribution [19, where the CaffarelliJerison-Kenig monotonicity formula has been extended to systems with variable coefficient. We point out that such contribution allows to slightly modify the proof of Theorem 1.4 to obtain uniform estimates 
up to the boundary, and then to obtain also the global regularity for solutions of systems with boundary conditions possibly depending on $\beta$.

Theorem 1.6. Under the assumptions of Theorem 1.4, let $\Omega$ be smooth and bounded, and let $\mathbf{u}_{\beta}$ satisfy the boundary conditions

$$
u_{i, \beta}=\varphi_{i, \beta} \quad \text { on } \partial \Omega
$$

in a weak sense, where $\varphi_{i, \beta} \in \operatorname{Lip}(\partial \Omega)$ are uniformly bounded in $\operatorname{Lip}(\partial \Omega)$. Then there exists $M>0$ independent of $\beta$ such that

$$
\left\|\mathbf{u}_{\beta}\right\|_{\operatorname{Lip}(\bar{\Omega})} \leq M
$$

In the proof of Theorem 1.3 we shall make use of new monotonicity formulae which are still not available for operators with variable coefficients. Therefore, for the moment the issue of the uniform regularity up to the boundary remains open, and will be investigated in future works.

6) Finally, we point out that local estimates as the ones in Theorem 1.3 and 1.4 can be used directly to obtain global uniform bounds in the case in which $\Omega=\mathbb{R}^{N}$. As an example, we have

Theorem 1.7. Let $\left\{\mathbf{u}_{\beta}\right\}$ be a family of $H_{\text {loc }}^{1}\left(\mathbb{R}^{N}\right)$ functions such that $\left\|\mathbf{u}_{\beta}\right\|_{L^{\infty}\left(\mathbb{R}^{N}\right)} \leq m$ for some $m>0$. Let us also assume that $\left\{\mathbf{u}_{\beta}\right\}$ solves either (1.4) or (1.6) in $\mathbb{R}^{N}$, under the respective assumptions. Then there exists $M>0$ independent of $\beta$ such that

$$
\left\|\mathbf{u}_{\beta}\right\|_{\operatorname{Lip}\left(\mathbb{R}^{N}\right)} \leq M .
$$

In the proofs of the main results, only for the sake of simplicity, we focus on the particular cases

$$
\begin{cases}-\Delta u_{i}=f_{i, \beta}\left(x, u_{i}\right)-\beta u_{i} \sum_{j \neq i} a_{i j} u_{j}^{2} & \text { in } \Omega \\ u_{i}>0 & \text { in } \Omega\end{cases}
$$

and

$$
\begin{cases}-\Delta u_{i}=f_{i, \beta}\left(x, u_{i}\right)-\beta u_{i} \sum_{j \neq i} u_{j} & \text { in } \Omega \\ u_{i}>0 & \text { in } \Omega .\end{cases}
$$

The reader can easily check that all the results hold in the generality specified by Theorems 1.3 and 1.4 , with the same proofs. Moreover, in the proof of Theorem 1.3 we assume that $\Omega \subset \mathbb{R}^{N}$ with $N \geq 3$. The case $N=2$ is in general easier to deal with, and it can be recovered extending the solution in one further dimension in a constant way.

1.5. Outline of the proofs (Hölder bounds vs. Lipschitz bounds). Here we give a rough idea of the proofs of the main theorems, which we think can serve as a guide towards the rest of the paper. Both the proofs of Theorem 1.3 and 1.4 proceed by contradiction and are based upon a blow-up analysis. In the following we focus on the variational setting, and we consider the case $f_{i, \beta} \equiv 0$ to simplify the notation. For any compact set $K \subset \subset \Omega$, we aim at showing that the Lipschitz semi-norm of $\mathbf{u}_{\beta}$ is bounded in $K$, uniformly in $\beta$. To this aim, we introduce a cut-off function $0 \leq \eta \leq 1$ such that $\eta \equiv 1$ in $K$ and $\operatorname{supp} \eta=: K^{\prime} \subset \subset \Omega$. If we prove that for some constant $C>0$ independent of $n$

$$
\sup _{x \in \Omega}\left|\nabla\left(\eta u_{i, \beta}\right)\right| \leq C \quad i=1, \ldots, k,
$$

then the desired result follows. Hence, we assume by contradiction that for a sequence $\beta_{n} \rightarrow+\infty$

$$
L_{n}:=\sup _{i} \sup _{x \in \Omega}\left|\nabla\left(\eta u_{i, \beta_{n}}\right)\right| \rightarrow+\infty ;
$$

up to relabelling and up to a subsequence $L_{n}:=\left|\nabla u_{1, \beta_{n}}\left(x_{n}\right)\right|$ for some $x_{n} \in K^{\prime}$. We introduce two blow-up sequences

$$
v_{i, n}(x):=\eta\left(x_{n}\right) \frac{u_{i, \beta_{n}}\left(x_{n}+r_{n} x\right)}{L_{n} r_{n}} \quad \text { and } \quad \bar{v}_{i, n}(x):=\frac{\left(\eta u_{i, \beta_{n}}\right)\left(x_{n}+r_{n} x\right)}{L_{n} r_{n}},
$$

where $r_{n} \rightarrow 0$ is chosen in such a way that $\sum_{i} \bar{v}_{i, n}(0)=1$. It is possible to check that both of them are defined in scaled domains exhausting $\mathbb{R}^{N}$. We point out that $\mathbf{v}_{n}$ satisfies an equation similar to that for 
$\mathbf{u}_{\beta_{n}}$ but, at a first glance, does not exhibit any property of compactness. On the other hand, it is not difficult to check that $\overline{\mathbf{v}}_{n}$ is uniformly convergent on compact sets to a limit function $\mathbf{v}$, but does not satisfy any reasonable equation. We shall prove that for every $r>0$

$$
\lim _{n \rightarrow \infty}\left\|\mathbf{v}_{n}-\overline{\mathbf{v}}_{n}\right\|_{L^{\infty}\left(B_{r}\right)}=0
$$

so that the convergence $\mathbf{v}_{n} \rightarrow \mathbf{v}$ in $\mathcal{C}_{\text {loc }}\left(\mathbb{R}^{N}\right)$ will follows by the convergence of $\overline{\mathbf{v}}_{n}$. We show then that the limit function $\mathbf{v}$ is non-constant and globally Lipschitz continuous in $\mathbb{R}^{N}$, and has only two non-trivial components, say $v_{1}$ and $v_{2}$; moreover, $\left(v_{1}, v_{2}\right)$ is non-negative and solves either the regular problem

$$
\begin{cases}-\Delta v_{1}=-v_{1} v_{2}^{2} & \text { in } \mathbb{R}^{N} \\ -\Delta v_{2}=-v_{1}^{2} v_{2} & \text { in } \mathbb{R}^{N}\end{cases}
$$

or the segregated one

$$
\begin{cases}-\Delta v_{1}=0 & \text { in }\left\{v_{1}>0\right\} \\ -\Delta v_{2}=0 & \text { in }\left\{v_{2}>0\right\} \\ v_{1} \cdot v_{2} \equiv 0 & \text { in } \mathbb{R}^{N} \\ -\Delta\left(v_{1}-v_{2}\right)=0 & \text { in } \mathbb{R}^{N} .\end{cases}
$$

The fact that $\mathbf{v}$ solves either a regular problem or a segregated one depends on the asymptotic relation of the sequences $\left(r_{n}\right)$ and $\left(L_{n}\right)$, which a priori is unknown. In both cases, a relevant fact which marks a striking difference with the present literature concerning uniform bounds in Hölder spaces is represented by the existence of globally Lipschitz continuous solutions for both the previous problems (in particular, in the second one the reader may simply consider $v=x_{1}^{+}, v=x_{1}^{-}$). On the contrary, as proved in [21], globally $\alpha$-Hölder continuous solutions does not exist for any $0<\alpha<1$. This means that in order to reach a contradiction we are not allowed to pass to the limit, but we have to argue directly on the blow-up sequence $\left\{\mathbf{v}_{n}\right\}$ and to prove a kind of approximate Liouville-type result, saying that in the previous setting, the sequence $\left\{\mathbf{v}_{n}\right\}$ cannot converge to a non-constant globally Lipschitz continuous limiting profile which solves 1.10 or 1.11). We will reach such a result by using Almgren type and Alt-Caffarelli-Friedman type monotonicity formulae in the variational setting, while in the symmetric one we make use of the celebrated Caffarelli-Jerison-Kenig monotonicity formula.

1.6. Plan of the paper. Section 2 concerns the blow-up analysis, which will be considered simultaneously for the variational case and for the symmetric one. In Section 3 we introduce the monotonicity formulae which will serves as main tools in the proof of Theorem 1.3 such proof will be the object of Section 4. In Section 5 we prove Theorem 1.4. We point out that, although all the monotonicity formulae will be applied either to the sequence $\left\{\mathbf{u}_{\beta_{n}}\right\}$, or to the blow-up sequence $\left\{\mathbf{v}_{n}\right\}$, in Section 3 we will state and prove them in higher generality, in order to provide the reader with results as flexible as possible.

Some notations. As usual, $B_{r}\left(x_{0}\right)$ denotes the open ball of centre $x_{0}$ and radius $r$. When $x_{0}=0$, we write simply $B_{r}$ instead of $B_{r}(0)$ for the sake of simplicity. The normal derivative and the tangential gradient of a funtion $u$ on a given surface are denoted by $\partial_{\nu}$ and $\nabla_{\theta}$ respectively. The capital letter $C$ stays for a positive constant which can differ from line to line.

\section{Asymptotic of the Blow up Sequence}

In this section we consider a system of type

$$
\begin{cases}-\Delta u_{i}=f_{i}\left(x, u_{i}\right)-\beta \sum_{j \neq i} a_{i j} u_{i} u_{j}^{q} & \text { in } \Omega \\ u_{i}>0 & \text { in } \Omega,\end{cases}
$$

and we address simultaneously the cases $q=2$ (variational interaction) and $q=1$ (Lotka-Volterra type interaction); in the latter situation, as specified in Theorem 1.4 we assume that $a_{i j}=1$ for every $i \neq j$. Without loss of generality, we suppose that $\Omega \supset B_{3}$, and we aim at proving the uniform Lipschitz bound in $B_{1}$. As in [14, 21, 28, 32], the problem of the uniform bound is tackled with the introduction of suitable blow-up sequences. Let $0 \leq \eta \leq 1$ be a smooth cut-off function such that $\eta=1$ in $B_{1}$ and $\eta=0$ in 
$\mathbb{R}^{N} \backslash B_{2}$. By definition, the family $\left\{\eta \mathbf{u}_{\beta}\right\}$ admits a uniform bound on the Lipschitz modulus of continuity if there exists a constant $C>0$ such that

$$
\sup _{i=1, \ldots, k} \sup _{\substack{x \neq y \\ x, y \in \overline{B_{2}}}} \frac{\left|\left(\eta u_{i, \beta}\right)(x)-\left(\eta u_{i, \beta}\right)(y)\right|}{|x-y|} \leq C .
$$

Since $\eta=1$ in $B_{1}$, this is sufficient to give the desired result. We first observe that, if $\beta$ is bounded, then a uniform bound of such kind does exist as a consequence of the regularity theory for elliptic equation (for which we refer, here and in the rest of the paper, to [18]): indeed the right hand side of 2.1 is in this case uniformly bounded in $L^{\infty}$, and the solution $\mathbf{u}_{\beta}$ is uniformly $\mathcal{C}^{1, \alpha}$-regular in the interior of $\Omega$, for every $\alpha<1$. Hence, we only need to consider the case $\beta \rightarrow+\infty$. We shall show that there exists $C>0$ such that

$$
\sup _{i=1, \ldots, k} \sup _{x \in \overline{B_{2}}}\left|\nabla\left(\eta u_{i, \beta}\right)(x)\right| \leq C \quad \text { for every } \beta \gg 1 .
$$

Let us assume by contradiction that this is not true and, consequently, that there exists a sequence $\beta_{n} \rightarrow+\infty$ and a corresponding sequence $\left\{\mathbf{u}_{\beta_{n}}\right\}$ such that

$$
L_{n}:=\sup _{i=1, \ldots, k} \sup _{x \in \overline{B_{2}}}\left|\nabla\left(\eta u_{i, \beta_{n}}\right)(x)\right| \rightarrow \infty \quad \text { as } n \rightarrow+\infty .
$$

Up to a relabelling, we may assume that the supremum is achieved for $i=1$ and at a point $x_{n} \in B_{2}$. Moreover, in the variational setting $q=2$, thanks to the local version of the main results in 21] (which have been proved in absence of the terms $f_{i, \beta_{n}}$ in Theorem 2.6 of [36], and which will appear in a more general setting in [28]), we may also choose a subsequence $\left\{\mathbf{u}_{\beta_{n}}\right\}$ which converges to some limiting profile $\mathbf{u}$ in $H^{1}\left(B_{2}\right)$ and in $\mathcal{C}^{0, \alpha}\left(B_{2}\right)$ for every $0<\alpha<1$. The contradiction argument is based upon two blow-up sequences:

$$
v_{i, n}(x):=\eta\left(x_{n}\right) \frac{u_{i, \beta_{n}}\left(x_{n}+r_{n} x\right)}{L_{n} r_{n}} \quad \text { and } \quad \bar{v}_{i, n}(x):=\frac{\left(\eta u_{i, \beta_{n}}\right)\left(x_{n}+r_{n} x\right)}{L_{n} r_{n}},
$$

both defined on the scaled domain $\left(\Omega-x_{n}\right) / r_{n} \supset\left(B_{3}-x_{n}\right) / r_{n}=: \Omega_{n}$. The functions $\overline{\mathbf{v}}_{n}$ are non-trivial in the subset $\left(B_{2}-x_{n}\right) / r_{n}=: \Omega_{n}^{\prime}$. We choose the scaling factor $r_{n}>0$ in such a way that

$$
\sum_{i=1}^{k} \bar{v}_{i, n}(0)=\sum_{i=1}^{k} \frac{\left(\eta u_{i, \beta_{n}}\right)\left(x_{n}\right)}{L_{n} r_{n}}=1 \quad \Longrightarrow \quad r_{n}=\sum_{i=1}^{k} \frac{\left(\eta u_{i, \beta_{n}}\right)\left(x_{n}\right)}{L_{n}} \rightarrow 0
$$

where the last conclusion follows by the uniform $L^{\infty}$ boundedness of the family $\left\{\mathbf{u}_{\beta}\right\}$. The following lemma focuses on some preliminary properties of the blow up sequences. At first, we define

$$
f_{i, n}(x, t):=r_{n} \frac{\eta\left(x_{n}\right)}{L_{n}} f_{i, \beta_{n}}\left(x_{n}+r_{n} x, t \frac{L_{n} r_{n}}{\eta\left(x_{n}\right)}\right) .
$$

Lemma 2.1. In the previous blow-up setting, the following assertions hold:

(1) $f_{i, n}\left(x, v_{i, n}(x)\right) \rightarrow 0$ uniformly in all $\Omega_{n}$ as $n \rightarrow \infty$;

(2) the scaled domains $\Omega_{n}$ and $\Omega_{n}^{\prime}$ exhaust $\mathbb{R}^{N}$, that is, $\Omega_{n}, \Omega_{n}^{\prime} \rightarrow \mathbb{R}^{N}$ as $n \rightarrow \infty$; moreover, $\Omega_{n} \supset$ $B_{1 / r_{n}}$ for every $n$;

(3) the sequence $\left\{\mathbf{v}_{n}\right\}$ satisfies

$$
-\Delta v_{i, n}=f_{i, n}\left(x, v_{i, n}\right)-M_{n} v_{i, n} \sum_{j \neq i} a_{i j} v_{j, n}^{q} \quad \text { in } \Omega_{n},
$$

where

$$
M_{n}:=\beta_{n}\left(\frac{L_{n}}{\eta\left(x_{n}\right)}\right)^{q} r_{n}^{2+q}
$$

(4) the sequence $\left\{\overline{\mathbf{v}}_{n}\right\}$ has uniformly bounded Lip-seminorm:

$$
\sup _{i=1, \ldots, k, k \neq y} \sup _{x \neq y} \frac{\left|\bar{v}_{i, n}(x)-\bar{v}_{i, n}(y)\right|}{|x-y|} \leq 1
$$

furthermore $\left|\nabla \bar{v}_{1, n}(0)\right|=1$, and $\left|\nabla v_{1, n}(0)\right| \rightarrow 1$ as $n \rightarrow \infty$; 
(5) there exists $\mathbf{v}$, globally Lipschitz continuous in $\mathbb{R}^{N}$ with Lipschitz constant equal to 1 , such that up to a subsequence both $\mathbf{v}_{n} \rightarrow \mathbf{v}$ and $\overline{\mathbf{v}}_{n} \rightarrow \mathbf{v}$ in $\mathcal{C}_{\text {loc }}\left(\mathbb{R}^{N}\right)$ as $n \rightarrow \infty$;

(6) There holds $\mathbf{v}_{n} \rightarrow \mathbf{v}$ in $H_{\text {loc }}^{1}\left(\mathbb{R}^{N}\right)$ as $n \rightarrow \infty$, and for any $r>0$ there exists $C>0$ such that

$$
\int_{B_{r}} M_{n} v_{i, n} \sum_{j \neq i} a_{i j} v_{j, n}^{q} \leq C \quad \text { for every } i .
$$

If $M_{n} \rightarrow+\infty$, then $v_{i, n} v_{j, n} \rightarrow 0$ as $n \rightarrow \infty$ for any $i \neq j$.

Proof. Points (1) and (3) are straightforward consequences of the definitions and of our assumptions.

(2) Since $0 \in \Omega_{n}^{\prime}$ for every $n$, to prove that $\Omega_{n}^{\prime} \rightarrow \mathbb{R}^{N}$ it is sufficient to check that $\operatorname{dist}\left(0, \partial \Omega_{n}^{\prime}\right) \rightarrow+\infty$ as $n \rightarrow \infty$. Firstly we observe that

$$
r_{n}=\sum_{i=1}^{k} \frac{\left(\eta u_{i, \beta_{n}}\right)\left(x_{n}\right)}{L_{n}} \leq \frac{\left\|\mathbf{u}_{\beta_{n}}\right\|_{L^{\infty}\left(B_{2}\right)}}{L_{n}} \eta\left(x_{n}\right) \leq \frac{m l}{L_{n}} \operatorname{dist}\left(x_{n}, \partial B_{2}\right),
$$

where $l$ denotes the Lipschitz constant of $\eta$. Therefore,

$$
\operatorname{dist}\left(0, \partial \Omega_{n}^{\prime}\right)=\frac{\operatorname{dist}\left(x_{n}, \partial B_{2}\right)}{r_{n}} \geq \frac{L_{n}}{m l} \rightarrow+\infty \quad \text { as } n \rightarrow \infty .
$$

The fact that $\Omega_{n} \supset B_{1 / r_{n}}$ follows by definition.

(4) The uniform bound on the Lipschitz seminorm of $\overline{\mathbf{v}}_{n}$, and the fact that $\left|\nabla \bar{v}_{1, n}(0)\right|=1$, are direct consequences of the definitions. Moreover

$$
\nabla \bar{v}_{1, n}(0)=\frac{u_{1, \beta_{n}}\left(x_{n}\right) \nabla \eta\left(x_{n}\right)}{L_{n}}+\frac{\eta\left(x_{n}\right) \nabla u_{1, \beta_{n}}\left(x_{n}\right)}{L_{n}}=o(1)+\nabla v_{1, n}(0)
$$

as $n \rightarrow \infty$.

(5) Let $r>0$. The sequence $\left\{\overline{\mathbf{v}}_{n}\right\}$ has a uniformly bounded Lipschitz seminorm in $\overline{B_{r}}$, and is uniformly bounded in 0 . Hence, by the Ascoli-Arzelà theorem, it is uniformly convergent (up to a subsequence) to some $\mathbf{v} \in \mathcal{C}\left(\overline{B_{r}}\right)$ having Lipschitz-seminorm bounded by 1 . To complete the proof, we show that $\mathbf{v}_{n}-\overline{\mathbf{v}}_{n} \rightarrow 0$ as $n \rightarrow \infty$ in $\mathcal{C}_{\text {loc }}\left(\mathbb{R}^{N}\right)$. To this aim, it is sufficient to observe that for any compact $K \subset \mathbb{R}^{N}$

$$
\sup _{x \in K}\left|v_{i, n}(x)-\bar{v}_{i, n}(x)\right|=\sup _{x \in K} \frac{u_{i, \beta_{n}}\left(x_{n}+r_{n} x\right)}{L_{n} r_{n}}\left|\eta\left(x_{n}\right)-\eta\left(x_{n}+r_{n} x\right)\right| \leq \sup _{x \in K} \frac{l m}{L_{n}}|x|,
$$

where we used the uniform boundedness of $\left\{\mathbf{u}_{n}\right\}$, and we recall that $l$ denotes the Lipschitz constant of $\eta$. Since $L_{n} \rightarrow+\infty$ and $K$ is compact, the desired result follows.

(6) As far as the estimate (2.5) is concerned, it is sufficient to test the equation for $v_{i, n}$ against a smooth cut-off function $0 \leq \varphi \leq 1$ such that $\varphi=1$ in $B_{r}$ and $\varphi=0$ in $\mathbb{R}^{N} \backslash B_{2 r}$ : we obtain

$$
\int_{B_{r}} M_{n} v_{i, n} \sum_{j \neq i} a_{i j} v_{j, n}^{q} \leq \int_{B_{2 r}}\left|f_{i, n}\left(x, v_{i, n}\right) \varphi+v_{i, n} \Delta \varphi\right| \leq C,
$$

where we used the boundedness of $\left\{\mathbf{v}_{n}\right\}$ in compact sets. Testing the equation for $v_{i, n}$ against $v_{i, n} \varphi^{2}$, we also deduce that

$$
\frac{1}{2} \int_{B_{r}}\left|\nabla v_{i, n}\right|^{2} \leq 2 \int_{B_{2 r}}|\nabla \varphi|^{2} v_{i, n}^{2}+\int_{B_{2 r}}\left(f_{i, n}\left(x, v_{i, n}\right) v_{i, n} \varphi^{2}-M_{n} v_{i, n}^{2} \sum_{j \neq i} a_{i j} v_{j, n}^{q} \varphi^{2}\right) \leq C,
$$

where, as before, we used the boundedness of $\left\{\mathbf{v}_{n}\right\}$ on compact sets and the 2.5. This implies that up to a subsequence $v_{i, n} \rightarrow v_{i}$ weakly in $H^{1}\left(B_{r}\right)$. In order to pass from the weak convergence to the strong one, we observe that since $\left\|v_{i, n}\right\|_{H^{1}\left(B_{r}\right)} \leq C$ independently of $n$, by replacing if necessary $r$ with a slightly smaller quantity we have also

$$
\int_{\partial B_{r}}\left|\nabla v_{i, n}\right|^{2} \leq C
$$


independently of $n$. Therefore, by testing the equation for $v_{i, n}$ against $\left(v_{i, n}-v_{i}\right)$ in $B_{r}$, we deduce that

$$
\begin{aligned}
\left|\int_{B_{r}} \nabla v_{i, n} \cdot \nabla\left(v_{i, n}-v_{i}\right)\right|= & \left|\int_{\partial B_{r}} \partial_{\nu} v_{i, n}\left(v_{i, n}-v_{i}\right)\right| \\
& \quad+\left|\int_{B_{r}} f_{i, n}\left(x, v_{i, n}\right)\left(v_{i, n}-v_{i}\right)-M_{n} v_{i, n} \sum_{j \neq i} a_{i j} v_{j, n}^{q}\left(v_{i, n}-v_{i}\right)\right| \\
\leq & \left(\int_{\partial B_{r}}\left|\partial_{\nu} v_{i, n}\right|+\int_{B_{r}}\left|f_{i, n}\left(x, v_{i, n}\right)\right|+M_{n} v_{i, n} \sum_{j \neq i} a_{i j} v_{j, n}^{q}\right)\left\|v_{i, n}-v_{i}\right\|_{L^{\infty}\left(B_{r}\right)} .
\end{aligned}
$$

Recalling that $v_{i, n} \rightarrow v_{i}$ uniformly in $B_{r}$ and that all the other terms are bounded, the desired result follows.

In the rest of this section, we aim at proving that the limit function $\mathbf{v}$ is non-constant and has exactly two non-trivial components. We have to distinguish between two cases, according to whether $\left(M_{n}\right)$ is bounded or not. In the former case, the function $\mathbf{v}$ will be shown to be non-constant as a result of the regularity theory for elliptic equations. In the latter one, the situation is more involved, and we shall make use of the following decay estimate, which allows to treat also more general interaction terms of type $u_{i}^{p} u_{j}^{q}$ with $p, q \geq 1$ falling under the assumptions of Theorems 1.3 or 1.4 .

Lemma 2.2. Let $x_{0} \in \mathbb{R}^{N}$ and $A, M, \delta, \rho>0$. Let $u \in H^{1}\left(B_{2 \rho}\left(x_{0}\right)\right) \cap \mathcal{C}\left(\overline{B_{2 \rho}\left(x_{0}\right)}\right)$ be a subsolution to

$$
\begin{cases}-\Delta u \leq-M u^{p}+\delta & \text { in } B_{2 \rho}\left(x_{0}\right) \\ u \leq A & \text { in } B_{2 \rho}\left(x_{0}\right)\end{cases}
$$

for some $p \geq 1$. Then there exists $C>0$, depending only on the dimension $N$, such that

$$
M u^{p}(x) \leq \frac{C A}{\rho^{2}}+\delta \quad \text { for every } x \in B_{\rho}\left(x_{0}\right) .
$$

Proof. Let $v \in H^{1}\left(B_{2 \rho}\left(x_{0}\right)\right)$ be a positive solution to

$$
\begin{cases}-\Delta v+M|v|^{p-1} v=0 & \text { in } B_{2 \rho}\left(x_{0}\right) \\ v=A & \text { on } \partial B_{2 \rho}\left(x_{0}\right)\end{cases}
$$

The existence of such function for any value of $M>0$ and $p \geq 1$ can be shown by the direct method of the calculus of variations. Moreover, the weak maximum principle implies that $v \leq A$ in $B_{2 \rho}\left(x_{0}\right)$. Let $\eta \in \mathcal{C}_{0}^{\infty}\left(B_{2 \rho}\left(x_{0}\right)\right)$ be a smooth cut-off function such that $\eta=1$ in $B_{3 \rho / 2}\left(x_{0}\right), 0 \leq \eta \leq 1$ and $|\Delta \eta| \leq C / \rho^{2}$. Testing the equation for $v$ against $\eta$ we obtain

$$
\int_{B_{3 \rho / 2}\left(x_{0}\right)} M v^{p} \leq \int_{B_{2 \rho}\left(x_{0}\right)} M v^{p} \eta=\int_{B_{2 \rho}\left(x_{0}\right)} \Delta v \eta=\int_{B_{2 \rho}\left(x_{0}\right)} v \Delta \eta \leq C A \rho^{N-2} .
$$

Let $y \in B_{\rho}\left(x_{0}\right)$. Since $v$ is subhamornic and $p \geq 1$, the mean value theorem gives

$$
M v(y)^{p} \leq M\left(\frac{1}{\left|B_{\rho / 2}(y)\right|} \int_{B_{\rho / 2}(y)} v\right)^{p} \leq \frac{1}{\left|B_{\rho / 2}(y)\right|} \int_{B_{\rho / 2}(y)} M v^{p} \leq \frac{C A}{\rho^{2}} .
$$

Let us now consider the auxiliary function $\bar{v}:=v+(\delta / M)^{1 / p}$. Trivially, one has $\bar{v}^{p} \geq v^{p}+\delta / M$, and thus

$$
\begin{cases}-\Delta \bar{v} \geq-M \bar{v}^{p}+\delta & \text { in } B_{2 \rho}\left(x_{0}\right) \\ \bar{v} \geq A & \text { on } \partial B_{2 \rho}\left(x_{0}\right) .\end{cases}
$$

Hence, $\bar{v}$ is a supersolution to 2.6 and the thesis follows applying the comparison principle.

Lemma 2.3. The limit function $\mathbf{v}$ is not constant. In particular, at least the first component $v_{1}$ is neither trivial nor constant.

Proof. As announced, we divide the proof according to properties of $\left(M_{n}\right)$. 
Case 1) $\left(M_{n}\right)$ is bounded. Since $\left\{\mathbf{v}_{n}\right\}$ is uniformly bounded in any compact set of $\mathbb{R}^{N}$, the sequence $\left\{\Delta v_{1, n}\right\}$ is uniformly bounded as well; by standard regularity theory for elliptic equations, we deduce that for every compact $K \subset \mathbb{R}^{N}$ there exists $C>0$ independent of $n$ such that $\left\|v_{1, n}\right\|_{\mathcal{C}^{1, \alpha}(K)} \leq C$. This implies that, up to a subsequence, the convergence of $v_{1, n}$ to $v_{1}$ takes place in $\mathcal{C}_{\text {loc }}^{1, \alpha}\left(\mathbb{R}^{N}\right)$ for any $0<\alpha<1$, so that in particular $\left|\nabla v_{1}(0)\right|=\lim _{n}\left|\nabla v_{1, n}(0)\right|=1$, and $\mathbf{v}$ cannot be a vector of constant functions.

Case 2) $M_{n} \rightarrow+\infty$. By the uniform bound (2.5) we infer that the limiting profile $\mathbf{v}$ is segregated: $v_{i} v_{j} \equiv 0$ in $\mathbb{R}^{N}$ for every $i \neq j$. Therefore, recalling that by the choice of $r_{n}$ we have $\sum_{i=1}^{k} v_{i, n}(0)=1$, there are two possibilities: either $v_{1}(0)=0$, or $v_{1}(0)=1$.

Assume at first that $v_{1}(0)=0$. Then there exists $h \neq 1$ such that $v_{h}(0)=1$, and by continuity of $\mathbf{v}$ it results that $v_{1} \equiv 0$ in an open neighbourhood of 0 . Moreover, $v_{h, n}(0) \geq 7 / 8$ for every $n$ sufficiently large. Thanks to points (4) and (5) of Lemma 2.1, we have

$$
\left|v_{h, n}(x)-v_{h, n}(0)\right| \leq\left|v_{h, n}(x)-\bar{v}_{h, n}(x)\right|+\left|\bar{v}_{h, n}(x)-\bar{v}_{h, n}(0)\right| \leq o(1)+|x| \leq o(1)+\frac{1}{2}
$$

as $n \rightarrow \infty$, for every $x \in B_{1 / 2}(0)$. Thus, whenever $n$ is sufficiently large, $v_{h, n} \geq 1 / 8$ in $B_{1 / 2}$. As a consequence, the equation for $v_{1, n}$ gives

$$
\begin{cases}-\Delta v_{1, n} \leq-C M_{n} v_{1, n}+\delta & \text { in } B_{1 / 2} \\ v_{1, n} \geq 0 & \text { in } B_{1 / 2} \\ v_{1, n} \leq A & \text { in } B_{1 / 2},\end{cases}
$$

where $\delta \geq \sup _{B_{1 / 2}(0)} f_{i, n}$ can be chosen independently of $n$, and the upper bound on $v_{1, n}$ in $B_{1 / 2}$ follows by the uniform boundedness of $\left\{\mathbf{v}_{n}\right\}$ in compact sets. By Lemma 2.2. we infer that $M_{n} v_{1, n} \leq C$ in $B_{1 / 4}$ independently of $n$. Therefore $\left|\Delta v_{1, n}(x)\right| \leq C$ for every $x \in B_{1 / 4}$, which implies that up to a subsequence $v_{1, n} \rightarrow v_{1}$ in $\mathcal{C}^{1}\left(B_{1 / 4}\right)$. In particular $\left|\nabla v_{1}(0)\right|=1$, in contradiction with the fact that $v_{1} \equiv 0$ in a neighbourhood of 0 . Thus, if $\left(M_{n}\right)$ is unbounded necessarily $v_{1}(0)=1$, and as a consequence the same argument described above provides $M_{n} v_{j, n} \leq C$ for every $x \in B_{1 / 4}$ and $j \neq 1$. Using again the uniform boundedness of the sequence $\left\{\mathbf{v}_{n}\right\}$ in $B_{1 / 4}$, we infer that $\left|\Delta v_{1, n}(x)\right| \leq C$ in $B_{1 / 4}$, and hence up to a subsequence $v_{1, n} \rightarrow v_{1}$ in $\mathcal{C}^{1}\left(B_{1 / 4}\right)$. In particular, by step (4) of Lemma 2.1 we have $\left|\nabla v_{1}(0)\right|=\lim _{n}\left|\nabla v_{1, n}(0)\right|=1$, which completes the proof.

Remark 2.4. The sequence $x_{n}$ is bounded and thus, up to a subsequence, converges to some $\bar{x} \in \overline{B_{2}}$. Following [14, 21, 28, it is possible to show that $\bar{x}$ has to be a free-boundary point, that is $\mathbf{u}(\bar{x})=0$ : indeed, if this is not the case, then there exists $i$ such that $u_{i}(\bar{x}) \geq C>0$. Using the local version of the main results in [21] (which hold for general systems, and for which we refer to [28]), $\mathbf{u}_{\beta_{n}} \rightarrow \mathbf{u}$ in $\mathcal{C}^{0, \alpha}\left(\overline{B_{2}}\right)$ for every $\alpha<1$, and in particular $u_{i, \beta_{n}}(x) \geq C / 2$ for every $x \in B_{2 \delta}(\bar{x})$ for some $\delta>0$ and $\beta_{n}$ sufficiently large. Reasoning as in Lemma 2.3 , this implies that $\mathbf{u}_{\beta_{n}} \rightarrow \mathbf{u}$ in $\mathcal{C}^{1, \alpha}\left(B_{\delta}(\bar{x})\right)$, a contradiction with the unboundedness of the gradient at $x_{n}$.

Before concluding the section, we report some further properties of the blow-up sequences and of the asymptotic behaviour of the quantities previously introduced.

Lemma 2.5. There exists $C>0$ such that $M_{n} \geq C$.

Proof. Let us assume by contradiction that there exists a subsequence $M_{n_{k}} \rightarrow 0$. By the previous results, the limiting function $\mathbf{v}$ is made of entire harmonic functions which are bounded from below, thus constant thanks to the Liouville theorem: this contradicts the fact that $\left|\nabla v_{1}(0)\right|=1$.

Lemma 2.6. Each limiting profile $\mathbf{v}$ contains at most two non trivial components.

The proof of the lemma is based upon the Alt-Caffarelli-Friedman monotonicity formula, as extended by Conti et al. in [14. We recall the results and some suitable generalizations whose proofs follow in a straightforward way and are thus omitted. 
Lemma 2.7 (Lemma 2.7 in [14]). Let $\mathbf{v} \in \mathcal{C}\left(\mathbb{R}^{N}\right) \cap H_{\text {loc }}^{1}\left(\mathbb{R}^{N}\right)$ be a vector of $k \geq 2$ non-trivial subharmonic functions such that $v_{i} v_{j} \equiv 0$ in $\mathbb{R}^{N}$ for every $i \neq j$, and there exists $x_{0} \in \mathbb{R}^{N}$ such that $v_{i}\left(x_{0}\right)=0$ for every $i$. Then there exists $\nu(k, N) \geq 1$ such that the quantity

$$
\Phi(r):=\prod_{i=1}^{k} \frac{1}{r^{2 \nu(k, N)}} \int_{B_{r}\left(x_{0}\right)} \frac{\left|\nabla v_{i}\right|^{2}}{|x|^{N-2}} \mathrm{~d} x
$$

is monotone non decreasing for $r>0$. If $k \geq 3$, then one can choose $\nu(k, N)>1$.

Corollary 2.8 (Hidden in Proposition 7.2 in [14]). Let $\mathbf{v}$ as in the previous lemma. If there exists $C>0$ such that

$$
|\mathbf{v}(x)| \leq C(1+|x|)
$$

then $k \leq 2$.

Lemma 2.9 (Lemma 2.7 in [14]). Let $\mathbf{v} \in \mathcal{C}\left(\mathbb{R}^{N}\right) \cap H_{\text {loc }}^{1}\left(\mathbb{R}^{N}\right)$ be a vector of $k \geq 2$ non-trivial positive functions, solutions to

$$
\Delta v_{i}=v_{i} \sum_{j \neq i} a_{i j} v_{j}^{q} \quad \text { in } \mathbb{R}^{N}
$$

for $q \geq 1$. There exists $\nu(k, N) \geq 1$ such that for every $\gamma<\nu$ there exists $\bar{r}>1$ such that the quantity

$$
\Phi(r):=\prod_{i=1}^{k} \frac{1}{r^{2 \gamma}} \int_{B_{r}}\left(\left|\nabla v_{i}\right|^{2}+v_{i}^{2} \sum_{j \neq i} a_{i j} v_{j}^{q}\right)|x|^{2-N}
$$

is monotone non decreasing for $r>\bar{r}$. If $k \geq 3$, then one can choose $\nu(k, N)>1$.

Corollary 2.10 (Hidden in Proposition 7.1 in [14]). Let $\mathbf{v}$ as in the previous lemma. If there exists $C>0$ such that

$$
|\mathbf{v}(x)| \leq C(1+|x|)
$$

then $k \leq 2$. If moreover $\mathbf{v}$ is non constant, then $k=2$.

Remark 2.11. For a detailed proof of Corollary 2.10, we refer to Corollary 1.14 in [29. In an analogue way, the reader can derive Corollary 2.8 starting from Lemma 2.7.

We conclude this section by summing up what we proved so far in the following statement.

Proposition 2.12. Let $\left\{\mathbf{u}_{\beta_{n}}\right\}$ satisfy the assumptions of Theorem 1.3 or 1.4 and assume that 2.3 holds. Then the sequences $\left\{\mathbf{v}_{n}\right\}$ and $\left\{\overline{\mathbf{v}}_{n}\right\}$ defined by (2.4) have the properties (1)-(6) of Lemma 2.1. There exists $C>0$ such that for every $i$

$$
v_{i}(x) \leq C(1+|x|) \quad \text { for every } x \in \mathbb{R}^{N},
$$

$\mathbf{v}$ is non-trivial and non-constant, and in particular $\left|\nabla v_{1}(0)\right|=1$. Moreover, $\mathbf{v}$ has at most 2 non-trivial components, say $v_{1}$ and $v_{2}, M_{n} \geq C>0$, and

- if $\left(M_{n}\right)$ is bounded, then

$$
\begin{cases}-\Delta v_{1}=-M_{\infty} v_{1} v_{2}^{q} & \text { in } \mathbb{R}^{N} \\ -\Delta v_{2}=-M_{\infty} v_{1}^{q} v_{2} & \text { in } \mathbb{R}^{N} \\ v_{1}, v_{2} \geq 0 & \text { in } \mathbb{R}^{N},\end{cases}
$$

where $M_{n} \rightarrow M_{\infty}$ as $n \rightarrow \infty$, and the convergence of $\mathbf{v}_{n}$ to $\mathbf{v}$ takes place in $\mathcal{C}_{\text {loc }}^{1, \alpha}\left(\mathbb{R}^{N}\right)$ for every $\alpha<1$.

- if $M_{n} \rightarrow+\infty$, then both $v_{1}$ and $v_{2}$ are subharmonic in $\mathbb{R}^{N}$, and

$$
\begin{cases}-\Delta v_{1}=0 & \text { in }\left\{v_{1}>0\right\} \\ -\Delta v_{2}=0 & \text { in }\left\{v_{2}>0\right\} \\ v_{1} \cdot v_{2} \equiv 0 & \text { in } \mathbb{R}^{N} \\ v_{1}, v_{2} \geq 0 & \text { in } \mathbb{R}^{N} .\end{cases}
$$




\section{Monotonicity FORMULÆ}

This section is devoted to some monotonicity formulae inspired by the Almgren frequency formula and the Alt-Caffarelli-Friedman monotonicity formula, which will be crucially employed in the proof of Theorem 1.3. Some of the following results are already present in the literature, but not in the following generality, and hence we prefer to also prove them for the sake of completeness. In Subsection 3.2 we will use the assumption $N \geq 3$. As already explained, the case $N=2$ can be treated extending planar solutions as spacial ones, but we point out that it would be also possible to face directly the planar problem. This would require a slightly different Alt-Caffarelli-Friedman monotonicity formula inspired by Lemma 9.2 in [11, which we prefer to omit.

3.1. Almgren monotonicity formulæ. Let us consider a smooth domain $\Omega \subset \mathbb{R}^{N}$, a compact set $K \subset \subset \Omega$ and a solution $\mathbf{u}=\left(u_{1}, \ldots, u_{k}\right) \in H^{1}(\Omega)$ of a generic problem of type $(1.8)$ satisfying the assumption 1.5 :

$$
\begin{cases}-\Delta u_{i}=f_{i}\left(x, u_{i}\right)-\beta \sum_{j \neq i} a_{i j} u_{i} u_{j}^{2} & \text { in } \Omega \\ u_{i} \geq 0 & \text { in } \Omega,\end{cases}
$$

and there exists $m, d>0$ such that

$$
\|\mathbf{u}\|_{L^{\infty}(\Omega)} \leq m \quad \text { and } \quad \max _{i} \sup _{0<s \leq m}\left|\frac{f_{i}(x, s)}{s}\right| \leq d .
$$

In what follows, all the constants that will appear depend on the choice of $m$ and $d$, which are considered throughout these preliminary results as fixed, but are independent on the choice of any other parameter (in particular, they are independent of $\beta>0$ ). The reason behind this observation is that, in the next section, we aim at using monotonicity formulae for sequence of solutions to (1.8) which verify the assumptions in a uniform way.

For $x_{0} \in K$ and $r>0$, we define

$$
\begin{aligned}
& \text { - } H\left(\mathbf{u}, x_{0}, r\right):=\frac{1}{r^{N-1}} \int_{\partial B_{r}\left(x_{0}\right)} \sum_{i=1}^{k} u_{i}^{2} \\
& \text { - } E\left(\mathbf{u}, x_{0}, r\right):=\frac{1}{r^{N-2}} \int_{B_{r}\left(x_{0}\right)} \sum_{i=1}^{k}\left|\nabla u_{i}\right|^{2}+2 \beta \sum_{1 \leq i<j \leq k} a_{i j} u_{i}^{2} u_{j}^{2}-\sum_{i=1}^{k} f_{i}\left(x, u_{i}\right) u_{i} \\
& \text { - } N\left(\mathbf{u}, x_{0}, r\right):=\frac{E\left(\mathbf{u}, x_{0}, r\right)}{H\left(\mathbf{u}, x_{0}, r\right)} \quad \text { (Almgren frequency function). }
\end{aligned}
$$

Lemma 3.1. Let $\mathbf{u}$ be a solution of (1.8) and (1.5). For $x_{0} \in K$ and $r>0$, we have

$$
\frac{\mathrm{d}}{\mathrm{d} r} H\left(\mathbf{u}, x_{0}, r\right)=\frac{2}{r^{N-1}} \int_{\partial B_{r}\left(x_{0}\right)} \sum_{i=1}^{k} u_{i} \partial_{\nu} u_{i}=2 \frac{E\left(\mathbf{u}, x_{0}, r\right)}{r} .
$$

Furthermore

$$
\begin{aligned}
\frac{\mathrm{d}}{\mathrm{d} r} E\left(\mathbf{u}, x_{0}, r\right) & =\frac{2}{r^{N-2}} \int_{\partial B_{r}\left(x_{0}\right)} \sum_{i=1}^{k}\left(\partial_{\nu} u_{i}\right)^{2}+\frac{(4-N)}{r^{N-1}} \beta \int_{B_{r}\left(x_{0}\right)} \sum_{1 \leq i<j \leq k} a_{i j} u_{i}^{2} u_{j}^{2} \\
& +\frac{1}{r^{N-1}} \int_{B_{r}\left(x_{0}\right)}\left[(N-2) \sum_{i=1}^{k} f_{i}\left(x, u_{i}\right) u_{i}+2 \sum_{i=1}^{k} f_{i}\left(x, u_{i}\right) \nabla u_{i} \cdot\left(x-x_{0}\right)\right] \\
& +\frac{1}{r^{N-2}} \beta \int_{\partial B_{r}\left(x_{0}\right)} \sum_{1 \leq i<j \leq k} a_{i j} u_{i}^{2} u_{j}^{2}-\frac{1}{r^{N-2}} \int_{\partial B_{r}\left(x_{0}\right)} \sum_{i=1}^{k} f_{i}\left(x, u_{i}\right) u_{i} .
\end{aligned}
$$


Proof. The equalities in (3.2) follow by direct computations. As far as the derivative of $E$ is concerned, we observe that

$$
\begin{aligned}
\frac{\mathrm{d}}{\mathrm{d} r} E\left(\mathbf{u}, x_{0}, r\right)= & \frac{\mathrm{d}}{\mathrm{d} r}\left(\frac{1}{r^{N-2}} \int_{B_{r}\left(x_{0}\right)} \sum_{i}\left|\nabla u_{i}\right|^{2}+2 \beta \sum_{i<j} a_{i j} u_{i}^{2} u_{j}^{2}\right)+\frac{(N-2)}{r^{N-1}} \int_{B_{r}\left(x_{0}\right)} \sum_{i} f_{i}\left(x, u_{i}\right) u_{i} \\
& -\frac{1}{r^{N-2}} \int_{\partial B_{r}\left(x_{0}\right)} \sum_{i} f_{i}\left(x, u_{i}\right) u_{i} .
\end{aligned}
$$

To compute the first term on the right hand side, letting $u_{i, r}(x):=u_{i}\left(x_{0}+r x\right)$, we have

$$
\begin{array}{r}
\frac{\mathrm{d}}{\mathrm{d} r}\left(\frac{1}{r^{N-2}} \int_{B_{r}\left(x_{0}\right)} \sum_{i}\left|\nabla u_{i}\right|^{2}+2 \beta \sum_{i<j} a_{i j} u_{i}^{2} u_{j}^{2}\right)=\frac{\mathrm{d}}{\mathrm{d} r}\left(\int_{B_{1}} \sum_{i}\left|\nabla u_{i, r}\right|^{2}+2 r^{2} \beta \sum_{i<j} a_{i j} u_{i, r}^{2} u_{j, r}^{2}\right) \\
=\int_{B_{1}} 2 \sum_{i} \nabla u_{i, r} \cdot \nabla\left(\partial_{r} u_{i, r}\right)+4 r \beta \sum_{i<j} a_{i j} u_{i, r}^{2} u_{j, r}^{2}+4 r^{2} \beta \sum_{i<j} a_{i j} u_{i, r} u_{j, r}\left(u_{j, r} \partial_{r} u_{i, r}+u_{i, r} \partial_{r} u_{j, r}\right) \\
=2 \int_{\partial B_{1}} \sum_{i} \partial_{r} u_{i, r} \partial_{\nu} u_{i, r}+\int_{B_{1}} 4 r \beta \sum_{i<j} a_{i j} u_{i, r}^{2} u_{j, r}^{2}+2 r^{2} \beta \sum_{i<j} a_{i j} u_{i, r} u_{j, r}\left(u_{j, r} \partial_{r} u_{i, r}+u_{i, r} \partial_{r} u_{j, r}\right) \\
+\int_{B_{1}} 2 r^{2} \sum_{i} f_{i}\left(x_{0}+r x, u_{i, r}\right) \partial_{r} u_{i, r} \\
=\frac{2}{r^{N-2}} \int_{\partial B_{r}\left(x_{0}\right)} \sum_{i}\left(\partial_{\nu} u_{i}\right)^{2}+\frac{1}{r^{N-1}} \int_{B_{r}\left(x_{0}\right)} 4 \beta \sum_{i<j} a_{i j} u_{i}^{2} u_{j}^{2}+\sum_{i<j} \nabla\left(u_{i}^{2} u_{j}^{2}\right) \cdot\left(x-x_{0}\right) \\
+\frac{2}{r^{N-1}} \int_{B_{r}\left(x_{0}\right)} \sum_{i} f_{i}\left(x, u_{i}\right) \nabla u_{i} \cdot\left(x-x_{0}\right) .
\end{array}
$$

After a further integration by parts, the thesis follows.

We recall the following formulation of the Poincaré inequality, which can be shown by a standard scaling argument.

Lemma 3.2 (Poincaré inequality). If $u \in H_{\mathrm{loc}}^{1}\left(\mathbb{R}^{N}\right)$, then the following inequality holds for any ball $B_{r}$ :

$$
\frac{1}{r^{N-2}} \int_{B_{r}}|\nabla u|^{2}+\frac{1}{r^{N-1}} \int_{\partial B_{r}} u^{2} \geq \frac{N-1}{r^{N}} \int_{B_{r}} u^{2} .
$$

Lemma 3.3. There exist two constants $\tilde{r}=\tilde{r}(m, d)>0$ and $\tilde{C}=\tilde{C}(m, d)>0$ such that

$$
N\left(\mathbf{u}, x_{0}, r\right)+1 \geq 0 \quad \text { and } \quad \frac{d}{d r} N\left(\mathbf{u}, x_{0}, r\right) \geq-\tilde{C}\left(N\left(\mathbf{u}, x_{0}, r\right)+1\right)
$$

for every $0<r \leq \tilde{r}, x_{0} \in K$.

Proof. Let us observe that, since by definition $H\left(\mathbf{u}, x_{0}, r\right) \geq 0$, the positivity of $N\left(\mathbf{u}, x_{0}, r\right)+1$ is equivalent to that of $E\left(\mathbf{u}, x_{0}, r\right)+H\left(\mathbf{u}, x_{0}, r\right)$. By the sublinearity of $f_{i}$, we have

$$
\begin{array}{r}
E\left(\mathbf{u}, x_{0}, r\right)+H\left(\mathbf{u}, x_{0}, r\right) \\
=\frac{1}{r^{N-2}} \int_{B_{r}\left(x_{0}\right)} \sum_{i}\left|\nabla u_{i}\right|^{2}+2 \beta \sum_{i<j} a_{i j} u_{i}^{2} u_{j}^{2}-\sum_{i} f_{i}\left(x, u_{i}\right) u_{i}+\frac{1}{r^{N-1}} \int_{\partial B_{r}\left(x_{0}\right)} \sum_{i} u_{i}^{2} \\
\geq \frac{1}{r^{N-2}} \int_{B_{r}\left(x_{0}\right)} \sum_{i}\left|\nabla u_{i}\right|^{2}-\frac{d r^{2}}{r^{N}} \int_{B_{r}\left(x_{0}\right)} \sum_{i} u_{i}^{2}+\frac{1}{r^{N-1}} \int_{\partial B_{r}\left(x_{0}\right)} \sum_{i} u_{i}^{2},
\end{array}
$$

and thus we can conclude with an application of the Poincaré inequality in Lemma 3.2, as long as $d r^{2} \leq d \tilde{r}^{2}<N-1$. 
We now pass to the proof of the monotonicity, first dealing with the function $N\left(\mathbf{u}, x_{0}, r\right)$. We compute the derivative of $N$ using Lemma 3.1. We have

$$
\begin{aligned}
& \frac{\mathrm{d}}{\mathrm{d} r} N\left(\mathbf{u}, x_{0}, r\right)=\frac{R\left(\mathbf{u}, x_{0}, r\right)}{H\left(\mathbf{u}, x_{0}, r\right)} \\
& \quad+\frac{2}{r^{2 N-3} H^{2}\left(\mathbf{u}, x_{0}, r\right)}\left[\left(\int_{\partial B_{r}\left(x_{0}\right)} \sum_{i}\left(\partial_{\nu} u_{i}\right)^{2}\right)\left(\int_{\partial B_{r}\left(x_{0}\right)} \sum_{i} u_{i}^{2}\right)-\left(\int_{\partial B_{r}\left(x_{0}\right)} \sum_{i} u_{i} \partial_{\nu} u_{i}\right)^{2}\right],
\end{aligned}
$$

where

$$
\begin{aligned}
R\left(\mathbf{u}, x_{0}, r\right):= & \frac{(4-N) \beta}{r^{N-1}} \int_{B_{r}\left(x_{0}\right)} \sum_{i<j} a_{i j} u_{i}^{2} u_{j}^{2} \\
& +\frac{1}{r^{N-1}} \int_{B_{r}\left(x_{0}\right)}\left[(N-2) \sum_{i} f_{i}\left(x, u_{i}\right) u_{i}+2 \sum_{i} f_{i}\left(x, u_{i}\right) \nabla u_{i} \cdot\left(x-x_{0}\right)\right] \\
& +\frac{\beta}{r^{N-2}} \int_{\partial B_{r}\left(x_{0}\right)} \sum_{i<j} a_{i j} u_{i}^{2} u_{j}^{2}-\frac{1}{r^{N-2}} \int_{\partial B_{r}\left(x_{0}\right)} \sum_{i} f_{i}\left(x, u_{i}\right) u_{i} \\
\geq & \frac{1}{r^{N-1}} \int_{B_{r}\left(x_{0}\right)}\left[(N-2) \sum_{i} f_{i}\left(x, u_{i}\right) u_{i}+2 \sum_{i} f_{i}\left(x, u_{i}\right) \nabla u_{i} \cdot\left(x-x_{0}\right)\right] \\
& -\frac{1}{r^{N-2}} \int_{\partial B_{r}\left(x_{0}\right)} \sum_{i} f_{i}\left(x, u_{i}\right) u_{i}=: R_{1}\left(\mathbf{u}, x_{0}, r\right) .
\end{aligned}
$$

Here we used the fact that $N \leq 4$. Thus, by the Cauchy-Schwarz inequality

$$
\frac{\mathrm{d}}{\mathrm{d} r} N\left(\mathbf{u}, x_{0}, r\right) \geq \frac{R_{1}\left(\mathbf{u}, x_{0}, r\right)}{H\left(\mathbf{u}, x_{0}, r\right)}
$$

for every $r>0$. We now estimate the remainder $R_{1}$, using the assumptions on the reaction terms $f_{i}$. For every $x_{0} \in K$ and $0<r \leq 1$ such that $B_{r}\left(x_{0}\right) \subset \subset K$, it results that

$$
\begin{array}{r}
\left|R_{1}\left(\mathbf{u}, x_{0}, r\right)\right| \leq \frac{1}{r^{N-1}} \int_{B_{r}\left(x_{0}\right)}\left[(N-2) d \sum_{i} u_{i}^{2}+2 d r \sum_{i} u_{i}\left|\nabla u_{i}\right|\right]+\frac{d}{r^{N-2}} \int_{\partial B_{r}\left(x_{0}\right)} \sum_{i} u_{i}^{2} \\
\leq \frac{d}{r^{N-2}} \int_{B_{r}\left(x_{0}\right)} \sum_{i}\left|\nabla u_{i}\right|^{2}+\frac{d r^{2}+(N-2) d r}{r^{N}} \int_{B_{r}\left(x_{0}\right)} \sum_{i} u_{i}^{2}+\frac{d r}{r^{N-1}} \int_{\partial B_{r}\left(x_{0}\right)} \sum_{i} u_{i}^{2} \\
\leq C(d, N)\left[\frac{1}{r^{N-2}} \int_{B_{r}\left(x_{0}\right)} \sum_{i}\left|\nabla u_{i}\right|^{2}+\frac{1}{r^{N}} \int_{B_{r}\left(x_{0}\right)} \sum_{i} u_{i}^{2}+\frac{1}{r^{N-1}} \int_{\partial B_{r}\left(x_{0}\right)} \sum_{i} u_{i}^{2}\right]
\end{array}
$$

The Poincaré inequality (Lemma 3.2 can be used in order to estimate the last term, showing that there exist $\tilde{C}, \tilde{r}>0$ such that

$$
\left|R_{1}\left(\mathbf{u}, x_{0}, r\right)\right| \leq \tilde{C}\left(E\left(\mathbf{u}, x_{0}, r\right)+H\left(\mathbf{u}, x_{0}, r\right)\right)
$$

for every $x_{0} \in K, 0<r \leq \tilde{r} \leq 1$. Coming back to 3.3 , we obtain the desired conclusion.

Remark 3.4. In the whole proof of Theorem 1.3, we use the assumption $N \leq 4$ only in the previous lemma. As we have already observed in the introduction, such an assumption can be dropped in absence of reaction terms $\left(f_{i, \beta} \equiv 0\right.$ for every $\left.i\right)$. In such case it is possible to replace the definition of $E\left(\mathbf{u}, x_{0}, r\right)$ with

$$
\tilde{E}\left(\mathbf{u}, x_{0}, r\right):=\frac{1}{r^{N-2}} \int_{B_{r}\left(x_{0}\right)} \sum_{i}\left|\nabla u_{i}\right|^{2}+\beta \sum_{i<j} u_{i}^{2} u_{j}^{2},
$$

proving an Almgren monotonicity formula for the function $\tilde{N}:=\tilde{E} / H$ independently on the dimension $N$ (we refer to Proposition 5.2 in [3] for the details). The rest of the proof of Theorem 1.3 can be adapted 
with minor changes.

We also point out that for $p \neq 1$ the condition $N \leq 4$ becomes $p \leq 2(1+1 / p)$.

Proposition 3.5. There exist $\tilde{r}=\tilde{r}(m, d)>0$ and $\tilde{C}=\tilde{C}(m, d)>0$ such that the functions

$$
r \mapsto\left(N\left(\mathbf{u}, x_{0}, r\right)+1\right) e^{\tilde{C} r} \quad \text { and } \quad r \mapsto\left(\frac{1}{r^{N-1}} \int_{\partial B_{r}\left(x_{0}\right)} u_{i}^{2}\right) e^{\tilde{C} r}
$$

are non-negative and monotone non-decreasing for $r \in(0, \tilde{r}]$, for every $x_{0} \in K$ and $i=1, \ldots, k$.

Proof. The first part is a straightforward consequence of Lemma 3.3. For the second part, we use assumption (1.5) and the Poincaré inequality (Lemma 3.2):

$$
\begin{aligned}
\frac{\mathrm{d}}{\mathrm{d} r}\left(\frac{1}{r^{N-1}} \int_{\partial B_{r}\left(x_{0}\right)} u_{i}^{2}\right) \geq \frac{2}{r^{N-1}} \int_{B_{r}\left(x_{0}\right)}\left|\nabla u_{i}\right|^{2}-\frac{2 d r}{r^{N}} \int_{B_{r}\left(x_{0}\right)} u_{i}^{2} \\
\geq \frac{2}{r} \frac{(N-1)-d r^{2}}{(N-1) r^{N-2}} \int_{B_{r}\left(x_{0}\right)}\left|\nabla u_{i}\right|^{2}-\frac{2 d r}{(N-1) r^{N-1}} \int_{\partial B_{r}\left(x_{0}\right)} u_{i}^{2} \geq-\frac{\tilde{C}^{\prime}}{r^{N-1}} \int_{\partial B_{r}\left(x_{0}\right)} u_{i}^{2},
\end{aligned}
$$

where the constant $\tilde{C}^{\prime}$ depends only on $d$ and on $N$, and the last inequality holds as long as $r<\tilde{r}^{\prime}(d, N)$ sufficiently small. Replacing, if necessary, $\tilde{C}$ and $\tilde{r}$ of Lemma 3.3 with $\max \left\{\tilde{C}, \tilde{C}^{\prime}\right\}$ and $\min \left\{\tilde{r}, \tilde{r}^{\prime}\right\}$, the thesis follows by a further integration.

We complete the first part of this subsection with two useful doubling properties.

Lemma 3.6. Let $\tilde{C}$ and $\tilde{r}$ be defined in the previous lemma.

(i) If there exist $0<\underline{r}<\bar{r}<\tilde{r}$ and $d>0$ such that $N(\mathbf{u}, 0, r) \leq d$ for every $\underline{r} \leq r \leq \bar{r}$, then

$$
r \mapsto \frac{H\left(\mathbf{u}, x_{0}, r\right)}{r^{2 d}} \quad \text { is monotone non-increasing for } \underline{r} \leq r \leq \bar{r} \text {. }
$$

(ii) If there exist $0<\underline{r}<\bar{r}<\tilde{r}$ and $\gamma>0$ such that $N(\mathbf{u}, 0, r) \geq \gamma$ for every $\underline{r} \leq r \leq \bar{r}$, then

$$
r \mapsto \frac{H\left(\mathbf{u}, x_{0}, r\right)}{r^{2 \gamma}} \quad \text { is monotone non-decreasing for } \underline{r} \leq r \leq \bar{r} \text {. }
$$

Proof. (i) By 3.2 we observe that

$$
\frac{\mathrm{d}}{\mathrm{d} r} \log H\left(\mathbf{u}, x_{0}, r\right)=\frac{2}{r} N\left(\mathbf{u}, x_{0}, r\right) \leq \frac{2 d}{r}
$$

for every $\underline{r} \leq r \leq \bar{r}$. By integrating, the thesis follows. The proof of $(i i)$ is analogue.

Almgren monotonicity formulae for segregated configurations. In [31 the authors introduced the sets $\mathcal{G}(\Omega)$ and $\mathcal{G}_{\text {loc }}(\Omega)$, classes of segregated vector valued functions sharing several properties with solutions of competitive systems, including a version of the Almgren monotonicity formula. We report Definition 1.2 in [31, which is of interest in the present setting.

Definition 3.7. For an open set $\Omega \subset \mathbb{R}^{N}$, we define the class $\mathcal{G}(\Omega)$ of non-trivial functions $\mathbf{0} \neq \mathbf{v}=$ $\left(v_{1}, \ldots, v_{k}\right)$ whose components are non-negative and locally Lipschitz continuous in $\Omega$, and such that the following properties holds:

- $v_{i} v_{j} \equiv 0$ in $\Omega$ for every $i \neq j$;

- for every $i$

$$
-\Delta v_{i}=f_{i}\left(x, v_{i}\right)-\mu_{i} \quad \text { in } \Omega \text { in distributional sense, }
$$

where $\mu_{i}$ is a non-negative Radon measure supported on the set $\partial\left\{v_{i}>0\right\}$, and $f_{i}: \Omega \times \mathbb{R}^{+} \rightarrow \mathbb{R}$ are $\mathcal{C}^{1}$ functions such that $\left|f_{i}(x, s)\right| \leq d|s|$, uniformly in $x$; 
- defining for $x_{0} \in \Omega$ and $r>0$ such that $B_{r}\left(x_{0}\right) \subset \Omega$ the function

$$
E\left(\mathbf{v}, x_{0}, r\right):=\frac{1}{r^{N-2}} \int_{B_{r}\left(x_{0}\right)} \sum_{i=1}^{k}\left|\nabla v_{i}\right|^{2}-\sum_{i=1}^{k} f_{i}\left(x, v_{i}\right) v_{i}
$$

we assume that $E$ is absolutely continuous as function of $r$ and

$$
\begin{aligned}
\frac{\mathrm{d}}{\mathrm{d} r} E\left(\mathbf{v}, x_{0}, r\right)= & \frac{1}{r^{N-2}} \int_{B_{r}\left(x_{0}\right)} \sum_{i=1}^{k}\left(\partial_{\nu} v_{i}\right)^{2}-\frac{1}{r^{N-2}} \int_{\partial B_{r}\left(x_{0}\right)} \sum_{i=1}^{k} f_{i}\left(x, u_{i}\right) u_{i} \\
& +\frac{1}{r^{N-1}} \int_{B_{r}\left(x_{0}\right)}\left[(N-2) \sum_{i=1}^{k} f_{i}\left(x, u_{i}\right) u_{i}+2 \sum_{i=1}^{k} f_{i}\left(x, u_{i}\right) \nabla u_{i} \cdot\left(x-x_{0}\right)\right] .
\end{aligned}
$$

For points $x_{0} \in\{\mathbf{v}=\mathbf{0}\}$, we define the multiplicity of $x_{0}$ as

$$
\sharp\left\{i=1, \ldots, k: \operatorname{meas}\left\{B_{r}\left(x_{0}\right) \cap\left\{v_{i}>0\right\}\right\}>0 \text { for every } r>0\right\} .
$$

We write that $\mathbf{v} \in \mathcal{G}_{\text {loc }}(\Omega)$ if $\mathbf{v} \in \mathcal{G}(K)$ for every compact set $K \subset \subset \Omega$.

Remark 3.8. The definition of $E$ in 3.1 and 3.4 are different, but we do not think that this can be source of misunderstanding, because the correct choice of $E$ is clearly determined by the vector valued function $\mathbf{v}$ which is considered. In the same spirit, we define the Almgren frequency function for elements of $\mathcal{G}(\Omega)$ as

$$
N\left(\mathbf{v}, x_{0}, r\right):=\frac{E\left(\mathbf{v}, x_{0}, r\right)}{H\left(\mathbf{v}, x_{0}, r\right)}
$$

with $H$ defined as in 3.1 .

We recall some known facts. The following are a monotonicity formula for functions of $\mathcal{G}(\Omega)$, and a lower estimate of $N\left(\mathbf{v}, x_{0}, 0^{+}\right)$for points $x_{0}$ on the free boundary $\{\mathbf{v}=\mathbf{0}\}$, for which we refer to Theorem 2.2 and Corollary 2.7 in 31 .

Theorem 3.9. Let $\mathbf{v} \in \mathcal{G}(\Omega)$ and let $K \subset \subset \Omega$. There exists $\tilde{r}^{\prime}, \tilde{C}^{\prime}$ depending only on $d$ and on the dimension $N$, such that for every $x_{0} \in K$ and $r \in\left(0, \tilde{r}^{\prime}\right]$ it results that $H\left(\mathbf{v}, x_{0}, r\right) \neq 0$, the function $N\left(\mathbf{v}, x_{0}, r\right)$ is absolutely continuous in $r$ and

$$
r \mapsto\left(N\left(\mathbf{v}, x_{0}, r\right)+1\right) e^{\tilde{C}^{\prime} r} \quad \text { is monotone non-decreasing. }
$$

Moreover, for every point of the free boundary $x_{0} \in\{\mathbf{v}=\mathbf{0}\}$ it results that $N\left(\mathbf{v}, x_{0}, 0^{+}\right) \geq 1$.

Remark 3.10. The fact that $H\left(\mathbf{v}, x_{0}, r\right) \neq 0$ for $r \in\left(0, \tilde{r}^{\prime}\right]$ and $x_{0} \in K$ is a unique continuation property for elements of $\mathcal{G}(\Omega)$ : indeed, if in an open subset of $\Omega$ we have $\mathbf{v} \equiv \mathbf{0}$, then $H\left(\mathbf{v}, x_{0}, r\right) \equiv 0$ for some $x_{0} \in \Omega$ and $r \in\left(r_{1}, r_{2}\right)$, in contradiction with the previous result.

The almost monotonicity formula for $N$ becomes a full monotonicity formula if $f_{i} \equiv 0$ for every $i$ (see Remark 2.4 in 31]). Moreover, thanks to a classification result due to [21] (see Step 6 in Proposition $3.9)$, the following holds.

Proposition 3.11. Let $\mathbf{v} \in \mathcal{G}(\Omega)$ with $f_{i} \equiv 0$ for every $i$. Then $r \mapsto N\left(\mathbf{v}, x_{0}, r\right)$ is non-decreasing. Moreover, it holds $N\left(\mathbf{v}, x_{0}, r\right) \equiv \sigma>0$ for $r \in(0, \bar{r}]$ if and only if $\mathbf{v}$ is a non-trivial homogeneous function of degree $\sigma$.

The relation between solutions of strongly competing systems and functions in $\mathcal{G}(\Omega)$ is clarified by the following statement, for which we refer to Theorem 8.1 in [31] in case $f_{i, \beta}\left(u_{i}\right):=\omega_{i} u_{i}^{3}-\lambda_{i, \beta} u_{i}$, and to [28] in a completely general setting.

Proposition 3.12. Let us a consider a sequence $\beta \rightarrow+\infty$, and let $\left\{\mathbf{u}_{\beta}\right\}$ be a corresponding sequence of solution to 1.8 in $\Omega$ satisfying 1.5 independently on $\beta$. Assume that

$$
f_{i, \beta} \rightarrow f_{i} \quad \text { in } \mathcal{C}_{\text {loc }}(\Omega \times[0, m])
$$


for some $f_{i} \in \mathcal{C}^{1}(\Omega \times[0, m])$, and that there exists $\mathbf{u}$ such that

$$
\mathbf{u}_{\beta} \rightarrow \mathbf{u} \quad \text { in } \mathcal{C}(\bar{\Omega}) \cap H^{1}(\Omega) .
$$

Then $\mathbf{u} \in \mathcal{G}(\Omega)$, and $N\left(\mathbf{u}_{\beta}, x, r\right) \rightarrow N(\mathbf{u}, x, r)$ for every $x \in \Omega$ and $r>0$ such that $B_{r}\left(x_{0}\right) \subset \subset \Omega$.

If $\mathbf{u}$ is as in the previous theorem, we write that $\mathbf{u}$ is a limiting profile of system 1.8 (as $\beta \rightarrow+\infty)$. A result which will be crucially employed in the rest of the section establishes the non occurrence of self-segregation for limiting profiles of strongly competing systems. This has been proved in Section 10 of $[15]$.

Theorem 3.13. Let $\mathbf{v} \in \mathcal{G}(\Omega)$ be a limiting profile of system 1 , and let $x_{0} \in\{\mathbf{v}=\mathbf{0}\}$. Then $x_{0}$ has multiplicity greater than or equal to 2 .

3.2. A perturbed Alt-Caffarelli-Friedman monotonicity formula. We introduce in a general setting an Alt-Caffarelli-Friedman monotonicity formula which has been proved for the first time in a specific situation in [36], Theorem 4.3; accordingly to the current section, here we consider the case $N \geq 3$.

We consider two components, say $u_{1}$ and $u_{2}$, of a solution $\mathbf{u}$ of system (1.8):

$$
\begin{cases}-\Delta u_{i}=f_{i}\left(x, u_{i}\right)-\beta \sum_{j \neq i} a_{i j} u_{i} u_{j}^{2} & \text { in } \Omega \\ u_{i}>0 & \text { in } \Omega .\end{cases}
$$

The ingredients of our result are the following:

$$
\begin{aligned}
\text { - } J_{1}(r):=\int_{B_{r}}\left(\left|\nabla u_{1}\right|^{2}+\beta a_{12} u_{1}^{2} u_{2}^{2}-u_{1} f_{1}\left(x, u_{1}\right)\right)|x|^{2-N} \\
\text { - } J_{2}(r):=\int_{B_{r}}\left(\left|\nabla u_{2}\right|^{2}+\beta a_{12} u_{1}^{2} u_{2}^{2}-u_{2} f_{2}\left(x, u_{2}\right)\right)|x|^{2-N} \\
\text { - } \gamma(t):=\sqrt{\left(\frac{N-2}{2}\right)^{2}+t-\frac{N-2}{2}} \\
\text { - } \Lambda_{1}(r):=\frac{r^{2} \int_{\partial B_{r}}\left|\nabla_{\theta} u_{1}\right|^{2}+\beta a_{12} u_{1}^{2} u_{2}^{2}-u_{1} f_{1}\left(x, u_{1}\right)}{\int_{\partial B_{r}} u_{1}^{2}} \\
\text { - } \Lambda_{2}(r):=\frac{r^{2} \int_{\partial B_{r}}\left|\nabla_{\theta} u_{2}\right|^{2}+\beta a_{12} u_{1}^{2} u_{2}^{2}-u_{2} f_{2}\left(x, u_{2}\right)}{\int_{\partial B_{r}} u_{2}^{2}} .
\end{aligned}
$$

Theorem 3.14. Let $\mathbf{u}$ be a solution of 1.8 and let $R>1, \lambda, \mu, \varepsilon>0$ be such that

(h0) $\varepsilon R^{2} \leq\left(\frac{N-2}{2}\right)^{2}$;

(h1) $J_{i}(r), \Lambda_{i}(r)>0$ for every $r \in(1, R)$, for $i=1,2$;

(h2) it holds

$$
\frac{1}{\lambda} \leq \frac{\int_{\partial B_{r}} u_{1}^{2}}{\int_{\partial B_{r}} u_{2}^{2}} \leq \lambda \quad \text { and } \quad \frac{1}{r^{N-1}} \int_{\partial B_{r}} u_{i}^{2} \geq \mu
$$

for every $r \in(1, R), i=1,2$;

(h3) $\left|f_{i}\left(x, u_{i}\right)\right| \leq \varepsilon u_{i}$ in $\Omega$ for $i=1,2$.

There exists a positive constant $C>0$, depending only on $\lambda, \mu$ and on the dimension $N$, such that

$$
r \mapsto \frac{J_{1}(r) J_{2}(r)}{r^{4}} \exp \left\{-C\left(\beta r^{2}\right)^{-1 / 4}+C \varepsilon r^{2}\right\} \quad \text { is monotone non-decreasing for } r \in(1, R) .
$$

Remark 3.15. For future convenience, we point out that the constant $C$ of the thesis is independent by the ends of the interval $(1, R)$.

The proof rests upon the following lemma, which can be seen as a Poincaré lemma on the sphere $\mathbb{S}^{N-1}$, $N \geq 3$ for two competing densities. This result is actually a generalization of Lemma 4.2 in 36 . 
For any $\lambda>0$, let

$$
H_{\lambda}:=\left\{(u, v) \in\left(H^{1}\left(\mathbb{S}^{N-1}\right)\right)^{2}: \int_{\mathbb{S}^{N-1}} u^{2}=1 \quad \text { and } \quad \int_{\mathbb{S}^{N-1}} v^{2}=\lambda\right\} .
$$

Lemma 3.16. Let us fix any $\bar{\lambda}>1$. There exists $C=C(N, \bar{\lambda})$ such that if

$$
\frac{1}{\bar{\lambda}}<\lambda<\bar{\lambda}, \quad k>0 \quad \text { and } \quad 0 \leq \varepsilon \leq\left(\frac{N-2}{2}\right)^{2}
$$

then

$$
\begin{aligned}
\min _{(u, v) \in H_{\lambda}} \gamma\left(\int_{\mathbb{S}^{N-1}}\left|\nabla_{\theta} u\right|^{2}+k u^{2} v^{2}-\varepsilon u^{2}\right)+\gamma\left(\frac{\int_{\mathbb{S}^{N-1}}\left|\nabla_{\theta} v\right|^{2}+k u^{2} v^{2}-\varepsilon v^{2}}{\int_{\mathbb{S}^{N-1}} v^{2}}\right) & \\
& \geq 2-C\left(\varepsilon+k^{-1 / 4}\right)
\end{aligned}
$$

Proof. It is straightforward to check that the estimate of the lemma is equivalent to related one for the functional

$$
J(u, v):=\gamma\left(\int_{\mathbb{S}^{N-1}}\left(\left|\nabla_{\theta} u\right|^{2}+k \lambda u^{2} v^{2}\right)-\varepsilon\right)+\gamma\left(\int_{\mathbb{S}^{N-1}}\left(\left|\nabla_{\theta} v\right|^{2}+k u^{2} v^{2}\right)-\varepsilon\right)
$$

considered on the $H^{1}$-weakly closed set $H_{1}$. First of all, we point out that such a minimization problem is well posed: indeed the domain of the function $t \mapsto \gamma(t)$ is given by the half line $t \geq-\left(\frac{N-2}{2}\right)^{2}$, and the restriction on $\varepsilon$ is sufficient to ensure the meaningfulness of (3.6). Moreover, for $k \geq 0$ the functional $J$ is coercive and lower-semicontinuous in the weak topology of $H^{1}\left(\mathbb{S}^{N-1}\right)$ : thus for any triplet $(\lambda, \varepsilon, k)$ that satisfies the assumptions, the minimization problem admits a solution. To conclude the lemma we only need to check the asymptotic expansion of the right hand side of $(3.6)$ for $\varepsilon$ small and $k$ large.

Let us a consider a sequence of triplets $\left(\lambda_{n}, \varepsilon_{n}, k_{n}\right)$ satisfying the assumptions and such that $\varepsilon_{n} \rightarrow 0$ and $k_{n} \rightarrow+\infty$, and for any such triplet let us consider a minimizer $\left(u_{n}, v_{n}\right)$ of the functional $J$. As $J(u, v)=J(|u|,|v|)$, it is not restrictive to assume that $u_{n}, v_{n} \geq 0$ in $\mathbb{S}^{N-1}$. Moreover, thanks to Lemma 4.1 in [36] it is possible to check that the functional $J$ is decreasing with respect to antipodal Steiner symmetrization rearrangements of the functions $\left(u_{n}, v_{n}\right)$, and thus we can also assume that the minimizer depends only on the angular coordinate on the sphere $\alpha \in[0, \pi]$, and that $u_{n}$ is decreasing while $v_{n}$ is increasing in $\alpha$. Let

$$
x_{n}=\int_{\mathbb{S}^{N-1}}\left(\left|\nabla_{\theta} u_{n}\right|^{2}+k_{n} \lambda_{n} u_{n}^{2} v_{n}^{2}\right)-\varepsilon_{n} \quad \text { and } \quad y_{n}=\int_{\mathbb{S}^{N-1}}\left(\left|\nabla_{\theta} v_{n}\right|^{2}+k_{n} u_{n}^{2} v_{n}^{2}\right)-\varepsilon_{n} .
$$

By the Lagrange multipliers rule, there exist $\mu_{1, n}, \mu_{2, n} \in \mathbb{R}$ such that

$$
\left\{\begin{array}{l}
-\Delta_{\theta} u_{n}=-k_{n}\left(\lambda_{n}+\frac{\gamma^{\prime}\left(y_{n}\right)}{\gamma^{\prime}\left(x_{n}\right)}\right) u_{n} v_{n}^{2}+\frac{\mu_{1, n}}{\gamma^{\prime}\left(x_{n}\right)} u_{n} \\
-\Delta_{\theta} v_{n}=-k_{n}\left(1+\frac{\gamma^{\prime}\left(x_{n}\right)}{\gamma^{\prime}\left(y_{n}\right)} \lambda_{n}\right) u_{n} v_{n}^{2}+\frac{\mu_{2, n}}{\gamma^{\prime}\left(y_{n}\right)} v_{n}
\end{array} \quad \text { in } \mathbb{S}^{N-1}\right.
$$

where $\Delta_{\theta}$ is the Laplace-Beltrami operator on the sphere $\mathbb{S}^{N-1}$. Since $\left(u_{n}, v_{n}\right)$ depends only on one angular coordinate, (3.7) simplifies as

$$
\left\{\begin{array}{l}
-\frac{\mathrm{d}^{2} u_{n}}{\mathrm{~d} \alpha^{2}}-(N-2) \cot \alpha \frac{\mathrm{d} u_{n}}{\mathrm{~d} \alpha}=-k_{n}\left(\lambda_{n}+\frac{\gamma^{\prime}\left(y_{n}\right)}{\gamma^{\prime}\left(x_{n}\right)}\right) u_{n} v_{n}^{2}+\frac{\mu_{1, n}}{\gamma^{\prime}\left(x_{n}\right)} u_{n} \\
-\frac{\mathrm{d}^{2} v_{n}}{\mathrm{~d} \alpha^{2}}-(N-2) \cot \alpha \frac{\mathrm{d} v_{n}}{\mathrm{~d} \alpha}=-k_{n}\left(1+\frac{\gamma^{\prime}\left(x_{n}\right)}{\gamma^{\prime}\left(y_{n}\right)} \lambda_{n}\right) v_{n} u_{n}^{2}+\frac{\mu_{2, n}}{\gamma^{\prime}\left(y_{n}\right)} v_{n}
\end{array} \quad \text { in }[0, \pi] .\right.
$$

Note that, with respect to Lemma 4.2 in [36], the presence of $\varepsilon_{n}$ is irrelevant for the characterization of $\left(u_{n}, v_{n}\right)$. As a consequence, it is possible to repeat step by step the proof of the quoted result, and to conclude that:

- the sequence $\left(J_{n}\left(u_{n}, v_{n}\right)\right)$ is bounded. Thus $\left\{\left(u_{n}, v_{n}\right)\right\}$ is bounded in $H^{1}\left(\mathbb{S}^{N-1}\right)$, and $\left(\gamma^{\prime}\left(x_{n}\right)\right)$, $\left(\gamma^{\prime}\left(y_{n}\right)\right)$ are bounded from above and from below by positive constants. Moreover, there exists $C>0$ independent of $n$ such that

$$
\int_{\mathbb{S}^{N-1}} k_{n} u_{n}^{2} v_{n}^{2} \leq C .
$$


- the sequences of the Lagrange multipliers $\left(\mu_{1, n}\right)$ and $\left(\mu_{2, n}\right)$ are bounded, and by a Brezis-Kato argument together with equation (3.7) and the $H^{1}$-boundedness, this implies that $\left\{\left(u_{n}, v_{n}\right)\right\}$ is bounded in $L^{\infty}\left(\mathbb{S}^{N-1}\right)$.

- There exists $\bar{\alpha}_{n} \in(0, \pi)$ such that $\left\{u_{n}>v_{n}\right\}=\left\{\theta \in \mathbb{S}^{N-1}: \alpha<\bar{\alpha}_{n}\right\},\left\{u_{n}=v_{n}\right\}=\left\{\theta \in \mathbb{S}^{N-1}\right.$ : $\left.\alpha=\bar{\alpha}_{n}\right\}$ and $\left\{u_{n}<v_{n}\right\}=\left\{\theta \in \mathbb{S}^{N-1}: \alpha>\bar{\alpha}_{n}\right\}$; up to multiplicative constants (depending on n) $u_{n} \rightarrow(\cos \alpha)^{+}, v_{n} \rightarrow(\cos \alpha)^{-}$in $H^{1}\left(\mathbb{S}^{N-1}\right) \cap \mathcal{C}\left(\mathbb{S}^{N-1}\right)$, and in particular $\bar{\alpha}_{n} \rightarrow \pi / 2$.

- There exists a constant $C>0$, independent of $\varepsilon_{n}$ and $k_{n}$, such that the Lipschitz norm of $\left(u_{n}, v_{n}\right)$ is smaller than $C$ (implied by a small modification of Lemma 2.4 in [2]).

- The following pointwise estimate holds uniformly in $n$ :

$$
u_{n} v_{n} \leq C k_{n}^{-1 / 2} \quad \text { in } \mathbb{S}^{N-1} .
$$

The decay estimate (3.8) implies that for every $\theta \in \mathbb{S}^{N-1}$, either $u_{n}(\theta) \leq C k_{n}^{-1 / 4}$ or $v_{n}(\theta) \leq C k_{n}^{-1 / 4}$. Let us introduce the functions $f_{n}=\left(u_{n}-v_{n}\right)^{+}$and $g_{n}=\left(u_{n}-v_{n}\right)^{-}$. As in [36, by (3.8)

$$
\int_{\mathbb{S}^{N-1}}\left|f_{n}-u_{n}\right|^{2} \leq C k_{n}^{-1 / 2},
$$

and

$$
\begin{aligned}
\int_{\mathbb{S}^{N-1}}\left|\nabla_{\theta} f_{n}\right|^{2} & \leq \int_{\left\{u_{n}>v_{n}\right\}}\left(\left|\nabla_{\theta} u_{n}\right|^{2}+\lambda_{n} k_{n} u_{n}^{2} v_{n}^{2}\right)+C k_{n}^{-1 / 4} \\
& \leq \int_{\left\{u_{n}>v_{n}\right\}}\left(\left|\nabla_{\theta} u_{n}\right|^{2}+\lambda_{n} k_{n} u_{n}^{2} v_{n}^{2}\right) \pm \varepsilon_{n}+C k_{n}^{-1 / 4} .
\end{aligned}
$$

Therefore

$$
\begin{aligned}
\frac{\int_{\mathbb{S}^{N-1}}\left|\nabla_{\theta} f_{n}\right|^{2}}{\int_{\mathbb{S}^{N-1}} f_{n}^{2}} & \leq \frac{\int_{\mathbb{S}^{N-1}}\left(\left|\nabla_{\theta} u_{n}\right|^{2}+\lambda_{n} k_{n} u_{n}^{2} v_{n}^{2}\right) \pm \varepsilon_{n}+C k_{n}^{-1 / 4}}{\int_{\mathbb{S}^{N-1}} u_{n}^{2}-C k_{n}^{-1 / 2}} \\
& =\frac{x_{n}+\varepsilon_{n}+C k_{n}^{-1 / 4}}{1-C k_{n}^{-1 / 2}} \leq x_{n}+\varepsilon_{n}+C k_{n}^{-1 / 4} .
\end{aligned}
$$

A similar estimate holds for $g_{n}$. By the monotonicity and the concavity of $\gamma$, we finally infer

$$
\begin{aligned}
& 2 \leq \gamma\left(\frac{\int_{\mathbb{S}^{N-1}}\left|\nabla_{\theta} f_{n}\right|^{2}}{\int_{\mathbb{S}^{N-1}} f_{n}^{2}}\right)+\gamma\left(\frac{\int_{\mathbb{S}^{N-1}}\left|\nabla_{\theta} g_{n}\right|^{2}}{\int_{\mathbb{S}^{N-1}} g_{n}^{2}}\right) \\
& \quad \leq \gamma\left(x_{n}+\varepsilon_{n}+C k_{n}^{-1 / 4}\right)+\gamma\left(y_{n}+\varepsilon_{n}+C k_{n}^{-1 / 4}\right) \leq \gamma\left(x_{n}\right)+\gamma\left(y_{n}\right)+C \varepsilon_{n}+C k_{n}^{-1 / 4},
\end{aligned}
$$

where the first inequality is a consequence of the fact that $f_{n}$ and $g_{n}$ have disjoint support (this is nothing but the well-known optimal partition problem of the sphere which serves as keystone in the proof of the original Alt-Caffarelli-Friedman monotonicity formula, see for instance [1, 9]).

Remark 3.17. In the proof of Lemma 4.2 in [36, the author makes use of the exponential decay estimate for solution of (1.8). The reader can easily check that such a decay can be replaced by the polynomial one proved in Lemma 2.2 . This allows to generalize the previous lemma, showing that for any $p \geq 1$, under the same assumptions on $\lambda, \varepsilon$ and $k$, there exists $C>0$ such that

$$
\begin{array}{r}
\min _{(u, v) \in H_{\lambda}} \gamma\left(\int_{\mathbb{S}^{N-1}}\left|\nabla_{\theta} u\right|^{2}+k u^{p+1} v^{p+1}-\varepsilon u^{2}\right)+\gamma\left(\frac{\int_{\mathbb{S}^{N-1}}\left|\nabla_{\theta} v\right|^{2}+k u^{p+1} v^{p+1}-\varepsilon v^{2}}{\int_{\mathbb{S}^{N-1}} v^{2}}\right) \\
\geq 2-C\left(\varepsilon+k^{-1 /(2 p+2)}\right) .
\end{array}
$$

Now a technical result.

Lemma 3.18. Let $i=1,2$, and let $r>0$ be such that $J_{i}(r)>0$ and $\Lambda_{i}(r)>0$. Then

$$
J_{i}(r) \leq \frac{r}{2 \gamma\left(\Lambda_{i}(r)\right)} \int_{\partial B_{r}}\left(\left|\nabla u_{i}\right|^{2}+\beta a_{12} u_{1}^{2} u_{2}^{2}-u_{i} f_{i}\left(x, u_{i}\right)\right)|x|^{2-N} .
$$


Proof. We consider $i=1$. By testing the equation for $u_{1}$ against $u_{1}|x|^{2-N}$ in $B_{r}$, we obtain

$$
\begin{aligned}
J_{1}(r) & =-\frac{1}{2} \int_{B_{r}} \nabla\left(u_{1}^{2}\right) \cdot \nabla\left(|x|^{2-N}\right)+\frac{1}{r^{N-2}} \int_{\partial B_{r}} u_{1} \partial_{\nu} u_{1} \\
& =\frac{1}{2} \int_{B_{r}} u_{1}^{2} \Delta\left(|x|^{2-N}\right)+\frac{1}{r^{N-2}} \int_{\partial B_{r}} u_{1} \partial_{\nu} u_{1}+\frac{N-2}{r^{N-1}} \int_{\partial B_{r}} u_{1}^{2} \\
& \leq \frac{1}{r^{N-2}} \int_{\partial B_{r}} u_{1} \partial_{\nu} u_{1}+\frac{N-2}{r^{N-1}} \int_{\partial B_{r}} u_{1}^{2}
\end{aligned}
$$

where the last inequality follows from the fact that $\Delta\left(|x|^{2-N}\right)=-C \delta$ for some dimensional constant $C>0$, where $\delta$ is the Dirac delta centred in 0 . Now, by Cauchy-Schwarz and Young inequalities, we have

$$
\begin{aligned}
\int_{\partial B_{r}} u_{1} \partial_{\nu} u_{1} & \leq\left(\int_{\partial B_{r}} u_{1}^{2}\right)^{1 / 2}\left(\int_{\partial B_{r}}\left(\partial_{\nu} u_{1}\right)^{2}\right)^{1 / 2} \\
& \leq \frac{\gamma\left(\Lambda_{1}(r)\right)}{2 r} \int_{\partial B_{r}} u_{1}^{2}+\frac{r}{2 \gamma\left(\Lambda_{1}(r)\right)} \int_{\partial B_{r}}\left(\partial_{\nu} u_{1}\right)^{2}
\end{aligned}
$$

where we used the fact that $\Lambda_{1}(r)>0$. Plugging this estimate in the previous chain of inequalities and using the definition of $\gamma$, we deduce that

$$
\begin{aligned}
J_{1}(r) & \leq \frac{1}{2 r^{N-1} \gamma\left(\Lambda_{1}(r)\right)}\left[\left(\gamma\left(\Lambda_{1}(r)\right)^{2}+(N-2) \gamma\left(\Lambda_{1}(r)\right)\right) \int_{\partial B_{r}} u_{1}^{2}+r^{2} \int_{\partial B_{r}}\left(\partial_{\nu} u_{1}\right)^{2}\right] \\
& \leq \frac{r^{2}}{2 r^{N-1} \gamma\left(\Lambda_{1}(r)\right)}\left[\int_{\partial B_{r}}\left(\left|\nabla_{\theta} u_{1}\right|^{2}+\beta a_{12} u_{1}^{2} u_{2}^{2}-u_{1} f_{1}\left(x, u_{1}\right)\right)+\int_{\partial B_{r}}\left(\partial_{\nu} u_{1}\right)^{2}\right]
\end{aligned}
$$

which is the desired result.

Proof of Theorem 3.14. Since for $r \in(1, R)$ both $J_{i}(r)$ and $\Lambda_{i}(r)$ are strictly positive, we can compute the logarithmic derivative of $J_{1}(r) J_{2}(r) / r^{4}$ and apply Lemma 3.18 , deducing that

$$
\begin{aligned}
\frac{\mathrm{d}}{\mathrm{d} r} \log \left(\frac{J_{1}(r) J_{2}(r)}{r^{4}}\right)=-\frac{4}{r}+ & \frac{\int_{\partial B_{r}}\left(\left|\nabla u_{1}\right|^{2}+\beta a_{12} u_{1}^{2} u_{2}^{2}-u_{1} f_{1}\left(x, u_{1}\right)\right)|x|^{2-N}}{\int_{B_{r}}\left(\left|\nabla u_{1}\right|^{2}+\beta a_{12} u_{1}^{2} u_{2}^{2}-u_{1} f_{1}\left(x, u_{1}\right)\right)|x|^{2-N}} \\
& +\frac{\int_{\partial B_{r}}\left(\left|\nabla u_{2}\right|^{2}+\beta a_{12} u_{1}^{2} u_{2}^{2}-u_{2} f_{2}\left(x, u_{2}\right)\right)|x|^{2-N}}{\int_{B_{r}}\left(\left|\nabla u_{2}\right|^{2}+\beta a_{12} u_{1}^{2} u_{2}^{2}-u_{2} f_{2}\left(x, u_{2}\right)\right)|x|^{2-N}} \\
\geq \frac{2}{r}\left(\gamma\left(\Lambda_{1}(r)\right)+\gamma\left(\Lambda_{2}(r)\right)-2\right) & -\frac{4}{r}+\frac{2}{r} \gamma\left(\frac{r^{2} \int_{\partial B_{r}}\left|\nabla_{\theta} u_{1}\right|^{2}+\beta a_{12} u_{1}^{2} u_{2}^{2}-\varepsilon u_{1}^{2}}{\int_{\partial B_{r}} u_{1}^{2}}\right) \\
\geq & +\frac{2}{r} \gamma\left(\frac{r^{2} \int_{\partial B_{r}}\left|\nabla_{\theta} u_{2}\right|^{2}+\beta a_{12} u_{1}^{2} u_{2}^{2}-\varepsilon u_{2}^{2}}{\int_{\partial B_{r}} u_{2}^{2}}\right)
\end{aligned}
$$

where we used assumption $(h 3)$ and the monotonicity of $\gamma$. The idea is now to apply Lemma 3.16 on the right hand side, and in order to do this we introduce

$$
u_{i, r}(x):=\frac{u_{i}(r x)}{\left(\frac{1}{r^{N-1}} \int_{\partial B_{r}} u_{1}^{2}\right)^{1 / 2}} \quad i=1,2
$$


we emphasize that both $u_{1}$ and $u_{2}$ are normalized with respect to the average of $u_{1}$. By direct computations

$$
\begin{aligned}
& \frac{r^{2} \int_{\partial B_{r}}\left|\nabla_{\theta} u_{1}\right|^{2}+\beta a_{12} u_{1}^{2} u_{2}^{2}-\varepsilon u_{1}^{2}}{\int_{\partial B_{r}} u_{1}^{2}}=\int_{\partial B_{1}}\left|\nabla_{\theta} u_{1, r}\right|^{2}+r^{2}\left(\frac{1}{r^{N-1}} \int_{\partial B_{r}} u_{1}^{2}\right) \beta a_{12} u_{1, r}^{2} u_{2, r}^{2}-\varepsilon r^{2} u_{1, r}^{2} \\
& \frac{r^{2} \int_{\partial B_{r}}\left|\nabla_{\theta} u_{2}\right|^{2}+\beta a_{12} u_{1}^{2} u_{2}^{2}-\varepsilon u_{2}^{2}}{\int_{\partial B_{r}} u_{2}^{2}}=\frac{\int_{\partial B_{1}}\left|\nabla_{\theta} u_{2, r}\right|^{2}+r^{2}\left(\frac{1}{r^{N-1}} \int_{\partial B_{r}} u_{1}^{2}\right) \beta a_{12} u_{1, r}^{2} u_{2, r}^{2}-\varepsilon r^{2} u_{2, r}^{2}}{\int_{\partial B_{1}} u_{2, r}^{2}} .
\end{aligned}
$$

Thanks to assumptions $(h 0)$ and $(h 2)$

$$
\begin{array}{r}
\varepsilon r^{2} \leq \varepsilon R^{2} \leq\left(\frac{N-2}{2}\right)^{2} \\
\int_{\partial B_{1}} u_{1, r}^{2}=1 \text { and } \quad \frac{1}{\lambda} \leq \int_{\partial B_{1}} u_{2, r}^{2} \leq \lambda \\
\frac{1}{r^{N-1}} \int_{\partial B_{r}} u_{1}^{2} \geq \mu
\end{array}
$$

for every $r \in(1, R)$ and $i=1,2$. Therefore we are in position to apply Lemma 3.16, obtaining

$$
\begin{aligned}
\frac{\mathrm{d}}{\mathrm{d} r} \log \left(\frac{J_{1}(r) J_{2}(r)}{r^{4}}\right) \geq & -\frac{4}{r}+ \\
& \frac{2}{r} \gamma\left(\int_{\partial B_{1}}\left|\nabla_{\theta} u_{1, r}\right|^{2}+r^{2} \mu \beta a_{12} u_{1, r}^{2} u_{2, r}^{2}-\varepsilon r^{2} u_{1, r}^{2}\right) \\
& +\frac{2}{r} \gamma\left(\frac{\int_{\partial B_{1}}\left|\nabla_{\theta} u_{2, r}\right|^{2}+r^{2} \mu \beta a_{12} u_{1, r}^{2} u_{2, r}^{2}-\varepsilon r^{2} u_{2, r}^{2}}{\int_{\partial B_{1}} u_{2, r}^{2}}\right) \\
\geq & -C \beta^{-1 / 4} r^{-3 / 2}-C \varepsilon r .
\end{aligned}
$$

By integrating, the thesis follows.

\section{INTERIOR LIPSCHITZ BOUND IN THE VARIATIONAL SETTING FOR $N \geq 3$}

In this section we complete the proof of Theorem 1.3 , obtaining a contradiction between the conclusions of the blow-up analysis, Proposition 2.12, and the assumption $L_{n} \rightarrow+\infty$. We recall that we are considering a sequence $\left\{\mathbf{u}_{\beta_{n}}\right\}$ of solutions to 1.8 satisfying the assumptions of Theorem 1.3, with $\beta_{n} \rightarrow+\infty$ :

$$
\begin{cases}-\Delta u_{i, \beta_{n}}=f_{i, \beta_{n}}\left(x, u_{i, \beta_{n}}\right)-\beta_{n} \sum_{j \neq i} a_{i j} u_{i, \beta_{n}} u_{j, \beta_{n}}^{2} & \text { in } \Omega \\ u_{i, \beta_{n}}>0 & \text { in } \Omega,\end{cases}
$$

there exist $m, d>0$ such that

$$
\left\|\mathbf{u}_{\beta_{n}}\right\|_{L^{\infty}(\Omega)} \leq m \quad \text { and } \quad \max _{i} \sup _{0<s \leq m}\left|\frac{f_{i, \beta_{n}}(x, s)}{s}\right| \leq d,
$$

and there exist functions $f_{i} \in \mathcal{C}^{1}(\Omega \times[0, m])$ such that

$$
f_{i, \beta_{n}} \rightarrow f_{i} \quad \text { in } \mathcal{C}_{\text {loc }}(\Omega \times[0, m]) \text { as } n \rightarrow \infty .
$$

Moreover, we are assuming that

$$
L_{n}:=\sup _{i=1, \ldots, k, k} \sup _{x \in B_{2}}\left|\nabla\left(\eta u_{i, \beta_{n}}\right)\right| \rightarrow+\infty .
$$

By (1.5) the quantities $\tilde{r}$ and $\tilde{C}$ in Proposition 3.5 can be chosen independently of $n$, and the following holds.

Proposition 4.1. There exists $\tilde{r}, \tilde{C}>0$, depending only on $m$ and on $d$, such that for every $n$ the functions

$$
r \mapsto\left(N\left(\mathbf{u}_{\beta_{n}}, x_{n}, r\right)+1\right) e^{\tilde{C} r} \quad \text { and } \quad r \mapsto\left(\frac{1}{r^{N-1}} \int_{\partial B_{r}\left(x_{n}\right)} u_{i, \beta_{n}}^{2}\right) e^{\tilde{C} r}
$$

are non-negative and monotone non-decreasing for $r \in(0, \tilde{r}]$. 
We introduce the quantity

$$
R_{\beta_{n}}:=\sup \left\{r \in(0, \tilde{r}):\left(N\left(\mathbf{u}_{\beta_{n}}, x_{n}, r\right)+1\right) e^{\tilde{C} r}<2-r\right\} .
$$

The role of $R_{\beta_{n}}$ will be clarified in the following. Before we establish some properties of the sequence $\left(R_{\beta_{n}}\right)$. Firstly, since for any fixed $n$ all the components $u_{i, \beta_{n}}$ are positive, $N\left(\mathbf{u}_{\beta_{n}}, x_{n}, 0^{+}\right)=0$ for every $n$, and hence $R_{\beta_{n}}>0$.

Lemma 4.2. $R_{\beta_{n}} \rightarrow 0$ as $n \rightarrow \infty$.

In order to prove the previous lemma, we need a result about uniform convergence.

Lemma 4.3. Let $g_{n} \in \mathcal{C}([0,1])$ be a sequence of monotone non-decreasing functions and let us assume that there exists $g \in \mathcal{C}([0,1])$ such that $g_{n} \rightarrow g$ pointwise in $[0,1]$. Then $g_{n} \rightarrow g$ uniformly in $[0,1]$.

Proof. We shall introduce two functions based on the partition of the set $[0,1]$ in $k$ equal sub-intervals For $k \in \mathbb{N}$ fixed, we let

$$
M_{n}^{k}(x):= \begin{cases}\sup _{j \geq n}\left(g_{j}(1 / k), g(1 / k)\right) & \text { if } x=0 \\ \sup _{j \geq n}\left(g_{j}(l / k), g(l / k)\right) & \text { if }(l-1) / k<x \leq l / k,\end{cases}
$$

and

$$
m_{n}^{k}(x):= \begin{cases}\inf _{j \geq n}\left(g_{j}((l-1) / k), g((l-1) / k)\right) & \text { if }(l-1) / k \leq x<l / k \\ \inf _{j \geq n}\left(g_{j}(1-1 / k), g(1-1 / k)\right) & \text { if } x=1 .\end{cases}
$$

From the monotonicity of the functions involved, we immediately obtain that

$$
m_{n}^{k}(x) \leq g_{j}(x) \leq M_{n}^{k}(x) \quad \text { and } \quad m_{n}^{k}(x) \leq g(x) \leq M_{n}^{k}(x) \quad \text { for every } x \in[0,1], k \in \mathbb{N}, j \geq n
$$

and

$$
\left|g_{j}(x)-g(x)\right| \leq M_{n}^{k}(x)-m_{n}^{k}(x) \quad \text { for every } x \in[0,1], k \in \mathbb{N}, j \geq n .
$$

On the other hand, by pointwise convergence for each $k \in \mathbb{N}$ there exists $N=N(k)$ such that

$$
\sup _{x}\left|g_{j}(x)-g(x)\right| \leq \sup _{x}\left(M_{N}^{k}(x)-m_{N}^{k}(x)\right) \leq 2 \underset{k}{\operatorname{osc} g} \quad \text { for } j \geq N(k),
$$

where $\operatorname{osc}_{k} g$ is the maximal oscillation of $g$ in each sub-interval of the considered $k$-partition. Since $g$ is uniformly continuous in $[0,1]$, the thesis follows by taking the limit in $k$.

We are now in a position to prove Lemma 4.2 .

Proof of Lemma 4.2. We have already observed in Remark 2.4 that up to a subsequence $\left\{\mathbf{u}_{\beta_{n}}\right\}$ converges in $\mathcal{C}\left(\overline{B_{2}}\right) \cap H^{1}\left(B_{2}\right)$, as $n \rightarrow \infty$, to a limiting profile $\mathbf{u}_{\infty} \in \mathcal{G}\left(\overline{B_{2}}\right)$, and also that $x_{n} \rightarrow x_{\infty} \in \overline{B_{2}} \cap\left\{\mathbf{u}_{\infty}=\mathbf{0}\right\}$. By the convergence

$$
r \mapsto\left(N\left(\mathbf{u}_{\infty}, x_{\infty}, r\right)+1\right) e^{\tilde{C} r} \quad \text { is monotone non-decreasing; }
$$

moreover, by Theorem $3.9, N\left(\mathbf{u}_{\infty}, x_{\infty}, 0^{+}\right) \geq 1$. Let us assume by contradiction that

$$
\limsup _{n \rightarrow \infty} R_{\beta_{n}}=R_{\infty}>0 \text {. }
$$

In light of the pointwise limit

$$
\lim _{n \rightarrow \infty}\left(N\left(\mathbf{u}_{\beta_{n}}, x_{n}, r\right)+1\right) e^{\tilde{C} r}=\left(N\left(\mathbf{u}_{\infty}, x_{\infty}, r\right)+1\right) e^{\tilde{C} r}
$$

valid for any $r \in(0, \tilde{r})$, and of the monotonicity of the involved functions, we can apply Lemma 4.3 to obtain that up to a subsequence

$$
\begin{aligned}
2 & >2-R_{\infty}=\lim _{n \rightarrow \infty} 2-R_{\beta_{n}} \geq \lim _{n \rightarrow \infty}\left(N\left(\mathbf{u}_{\beta_{n}}, x_{n}, R_{\beta_{n}}\right)+1\right) e^{\tilde{C} R_{\beta_{n}}} \\
& =\left(N\left(\mathbf{u}_{\infty}, x_{\infty}, R_{\infty}\right)+1\right) e^{\tilde{C} R_{\infty}} \geq\left(N\left(\mathbf{u}_{\infty}, x_{\infty}, 0^{+}\right)+1\right) \geq 2
\end{aligned}
$$

a contradiction. Here we used the fact that $x_{\infty} \in\left\{\mathbf{u}_{\infty}=\mathbf{0}\right\}$, so that by Theorem 3.9 we have $N\left(\mathbf{u}_{\infty}, x_{\infty}, 0^{+}\right) \geq 1$. 
The previous results can be translated in terms of the elements of the blow-up sequence $\left\{\mathbf{v}_{n}\right\}$ (for the reader's convenience, we recall that $\left\{\mathbf{v}_{n}\right\}$ has been defined in (2.4)).

Lemma 4.4. Let $\tilde{r}, \tilde{C}$ be defined in Proposition 4.1. For every $n \in \mathbb{N}$, the functions

$$
r \mapsto\left(N\left(\mathbf{v}_{n}, 0, r\right)+1\right) e^{\tilde{C} r_{n} r} \quad \text { and } \quad r \mapsto\left(\frac{1}{r^{N-1}} \int_{\partial B_{r}} v_{i, n}^{2}\right) e^{\tilde{C} r_{n} r}
$$

are non-negative and monotone non-decreasing for $r \in\left(0, \tilde{r} / r_{n}\right]$.

For the proof it is sufficient to check that

$$
\begin{array}{ll}
\text { - } & E\left(\mathbf{v}_{n}, 0, r\right)=\frac{\eta^{2}\left(x_{n}\right)}{L_{n}^{2} r_{n}^{2}} E\left(\mathbf{u}_{\beta_{n}}, x_{n}, r_{n} r\right) \\
\text { - } H\left(\mathbf{v}_{n}, 0, r\right)=\frac{\eta^{2}\left(x_{n}\right)}{L_{n}^{2} r_{n}^{2}} H\left(\mathbf{u}_{\beta_{n}}, x_{n}, r_{n} r\right) \\
\text { - } N\left(\mathbf{v}_{n}, 0, r\right)=N\left(\mathbf{u}_{\beta_{n}}, x_{n}, r_{n} r\right)
\end{array}
$$

for every $0<r \leq \tilde{r} / r_{n}$.

In the following lemma we enforce the conclusion of Proposition 2.12, showing that not only the limiting profile $\mathbf{v}$ of the blow-up sequence has at least one and at most two non-trivial components $v_{1}$ and $v_{2}$, but that both $v_{1}$ and $v_{2}$ are non-trivial and non-constant in the ball $B_{2}$.

Lemma 4.5. There exists $C>0$ independent of $n$ such that

$$
\frac{1}{r^{N-1}} \int_{\partial B_{r}} v_{i, n}^{2} \geq C
$$

for every $r \in\left[2, \tilde{r} / r_{n}\right]$ and $i=1,2$. In particular, both $v_{1, n}$ and $v_{2, n}$ are non-trivial and non-constant in $B_{r}$ for every $r \in\left[2, \tilde{r} / r_{n}\right]$.

Proof. By Lemma 4.4, we infer that for $r \in\left[2, \tilde{r} / r_{n}\right]$ it holds

$$
\frac{1}{r^{N-1}} \int_{\partial B_{r}} v_{i, n}^{2} \geq\left(\frac{1}{2^{N-1}} \int_{\partial B_{2}} v_{i, n}^{2}\right) e^{\tilde{C} r_{n}\left(2-\tilde{r} / r_{n}\right)},
$$

so that it is sufficient to show that there exists $C>0$ independent of $n$ such that

$$
\frac{1}{2^{N-1}} \int_{\partial B_{2}} v_{i, n}^{2} \geq C \quad \text { for } i=1,2
$$

We separate the prove according to whether $\left(M_{n}\right)$ is bounded or not.

Case $i)\left(M_{n}\right)$ is bounded.

Assume by contradiction that the 4.3 is not true. By Proposition 2.12, $\mathbf{v}_{n} \rightarrow \mathbf{v}$ and $\left(v_{1}, v_{2}\right)$ solves 2.7 . Moreover, $v_{1}(0)>0$ and, by subharmonicity, this implies that there exists $C>0$ such that

$$
\frac{1}{2^{N-1}} \int_{\partial B_{2}} v_{1}^{2} \geq 2 C \Longrightarrow \frac{1}{2^{N-1}} \int_{\partial B_{2}} v_{1, n}^{2} \geq C
$$

for $n$ sufficiently large. As a consequence, using the subharmonicity of $v_{2}$

$$
\int_{\partial B_{2}} v_{2, n}^{2} \rightarrow 0 \quad \Longrightarrow \quad v_{2} \equiv 0 \quad \text { in } B_{2} .
$$

By the strong maximum principle, this means that $v_{2} \equiv 0$ in $\mathbb{R}^{N}$, and thus $v_{1}$ is an entire, harmonic, non-constant and positive function, a contradiction.

Case $i$ i) $M_{n} \rightarrow+\infty$.

Arguing as in the first step, we deduce that $v_{2} \equiv 0$ in $B_{2}$, and by Proposition 3.12 we know that $\mathbf{v} \in \mathcal{G}_{\text {loc }}\left(\mathbb{R}^{N}\right)$. By the unique continuation property given by the Almgren monotonicity formula (see Remark 3.10 this implies that $v_{1}>0$ in $B_{2}$, and as a consequence $v_{1}$ is harmonic therein. To sum up, $v_{1}$ is a positive harmonic function in $B_{2}$ such that $v_{1}(0)=1$ and $\left|\nabla v_{1}(0)\right|=1$. Let $\theta \in \mathbb{S}^{N-1}$ be such that $\partial_{\theta} v_{1}=-1$. Since $\partial_{\theta} v_{1}$ is in turn harmonic in $B_{2}$, by the minimum principle $\inf _{B_{r}} \partial_{\theta} v_{1} \leq-1$ for any $r \in(0,2)$, and this immediately implies that $v_{1}$ changes sign in $B_{2}$, a contradiction. 
We introduce the counterpart of the radius $R_{\beta_{n}}$ in the blow-up setting, as

$$
\bar{r}_{n}:=\frac{R_{\beta_{n}}}{r_{n}}=\sup \left\{r \in\left(0, \frac{\tilde{r}}{r_{n}}\right):\left(N\left(\mathbf{v}_{n}, 0, r\right)+1\right) e^{\tilde{C} r_{n} r}<2-r_{n} r\right\} .
$$

By Lemma 4.2, we deduce that $r_{n} \bar{r}_{n} \rightarrow 0$ as $n \rightarrow \infty$.

The value $\bar{r}_{n}$ will play a crucial role in the forthcoming argument. The idea is the following: for $r<\bar{r}_{n}$ it results $N\left(\mathbf{v}_{n}, 0, r\right) \leq 1$, and we shall show that consequently $\mathbf{v}_{n}$ exhibits a linear behaviour; on the contrary if $r>\bar{r}_{n}$, then $N\left(\mathbf{v}_{n}, 0, r\right) \gtrsim 1$, so that $\mathbf{v}_{n}$ is morally superlinear. We will prove, in the superlinear range $\left(\bar{r}_{n}, \tilde{r} / r_{n}\right)$ the function $\left(E\left(\mathbf{v}_{n}, 0, r\right)+H\left(\mathbf{v}_{n}, 0, r\right)\right) / r^{2}$ is almost non-decreasing, uniformly in $n$. If the sequence $\left(\bar{r}_{n}\right)$ is bounded from above, this easily leads to a contradiction with Proposition 2.12. A more delicate situation takes place when $\bar{r}_{n} \rightarrow+\infty$, that is, $\bar{r}_{n}$ is an intermediate scale between the microscopic setting $r \leq R<+\infty$ and the macroscopic scale $\tilde{r} / r_{n} \rightarrow+\infty$. In such a situation the function $\mathbf{v}_{n}$ transits from the linear behaviour to the superlinear one at the threshold $\bar{r}_{n} \rightarrow+\infty$, with $\bar{r}_{n} r_{n} \rightarrow 0$. In the linear range $\left[2, \bar{r}_{n}\right]$, we shall derive a uniform-in- $n$ perturbed version of the Alt-Caffarelli-Friedman monotonicity formula. In the superlinear range, the almost monotonicity of the function $\left(E\left(\mathbf{v}_{n}, 0, r\right)+H\left(\mathbf{v}_{n}, 0, r\right)\right) / r^{2}$ holds. A delicate part of the proof consist in showing that a suitable combination of these results, which considered separately do not lead to any conclusive argument, permits to reach a contradiction also in this case.

In the next lemma we prove that the function $\left(E\left(\mathbf{v}_{n}, 0, r\right)+H\left(\mathbf{v}_{n}, 0, r\right)\right) / r^{2}$ is almost monotone beyond the threshold $\bar{r}_{n}$. Note that by the definition of $\tilde{r}$ the numerator is positive in the whole interval $\left[0, \tilde{r} / r_{n}\right]$. Let us introduce

$$
\varphi_{n}(r):=2 \int_{\bar{r}_{n}}^{r}\left(2 \frac{e^{-\tilde{C} r_{n} t}-1}{t}-\frac{1}{t} r_{n} \bar{r}_{n} e^{-\tilde{C} r_{n} t}\right) \mathrm{d} t .
$$

Remark 4.6. Not only $\varphi_{n}$ is well defined for $r \in\left[\bar{r}_{n}, \tilde{r} / r_{n}\right]$, but it is bounded independently of $n$ in such interval:

$$
\begin{aligned}
\left|\varphi_{n}(r)\right| & \leq 4 \int_{\bar{r}_{n}}^{\tilde{r} / r_{n}} \frac{1-e^{-\tilde{C} r_{n} t}}{t} \mathrm{~d} t+2 r_{n} \bar{r}_{n} \int_{\bar{r}_{n}}^{\tilde{r} / r_{n}} \frac{e^{-\tilde{C} r_{n} t}}{t} \mathrm{~d} t \\
& \leq 4 \int_{\bar{r}_{n}}^{\tilde{r} / r_{n}} \tilde{C} r_{n} \mathrm{~d} t+2 \bar{r}_{n} r_{n} \int_{\bar{r}_{n}}^{\tilde{r} / r_{n}} \frac{\mathrm{d} t}{t} \leq 4 \tilde{C} \tilde{r}+2 \bar{r}_{n} r_{n}\left(|\log \tilde{r}|+\left|\log \left(\bar{r}_{n} r_{n}\right)\right|\right) \leq C,
\end{aligned}
$$

with $C$ independent of $n$.

Lemma 4.7. For every $n$, the function

$$
r \mapsto \frac{E\left(\mathbf{v}_{n}, 0, r\right)+H\left(\mathbf{v}_{n}, 0, r\right)}{r^{2}} e^{\tilde{C} r_{n} r-\varphi_{n}(r)} \quad \text { is monotone non-decreasing for } r \in\left[\bar{r}_{n}, \frac{\tilde{r}}{r_{n}}\right] \text {. }
$$

Proof. If $r \in\left[\bar{r}_{n}, \tilde{r} / r_{n}\right]$, then by the Almgren monotonicity formula

$$
N\left(\mathbf{v}_{n}, 0, r\right)-1 \geq 2\left(e^{-\tilde{C} r_{n} r}-1\right)-r_{n} \bar{r}_{n} e^{-\tilde{C} r_{n} r}
$$

As a consequence, recalling the expression $\sqrt{3.2}$ of the derivative of $H$, we have

$$
\frac{\mathrm{d}}{\mathrm{d} r} \log \left(\frac{H\left(\mathbf{v}_{n}, 0, r\right)}{r^{2}}\right)=\frac{2}{r}\left(N\left(\mathbf{v}_{n}, 0, r\right)-1\right) \geq \frac{4}{r}\left(e^{-\tilde{C} r_{n} r}-1\right)-\frac{2}{r} r_{n} \bar{r}_{n} e^{-\tilde{C} r_{n} r} .
$$

By integrating, we deduce that the function

$$
r \mapsto \frac{H\left(\mathbf{v}_{n}, 0, r\right)}{r^{2}} e^{-\varphi_{n}(r)} \quad \text { is monotone non-decreasing for } r \in\left[\bar{r}_{n}, \tilde{r} / r_{n}\right] .
$$

To conclude, it is sufficient to observe that by Lemma 4.4

$$
\begin{aligned}
\frac{\mathrm{d}}{\mathrm{d} r} \log \left(\frac{E\left(\mathbf{v}_{n}, 0, r\right)+H\left(\mathbf{v}_{n}, 0, r\right)}{r^{2}} e^{\tilde{C} r_{n} r-\varphi_{n}(r)}\right) & \\
= & \frac{\mathrm{d}}{\mathrm{d} r} \log \left(\left(N\left(\mathbf{v}_{n}, 0, r\right)+1\right) e^{\tilde{C} r_{n} r}\right)+\frac{\mathrm{d}}{\mathrm{d} r} \log \left(\frac{H\left(\mathbf{v}_{n}, 0, r\right)}{r^{2}} e^{-\varphi_{n}(r)}\right) \geq 0
\end{aligned}
$$


for $\bar{r}_{n} \leq r \leq \tilde{r} / r_{n}$.

As a first consequence we can show that $\left(\bar{r}_{n}\right)$ cannot be bounded.

Lemma 4.8. It holds $\bar{r}_{n} \rightarrow+\infty$ as $n \rightarrow \infty$.

Proof. Suppose, by contradiction, that up to a subsequence $\bar{r}_{n} \leq \bar{r}$ for some $\bar{r}>0$. By the convergence of $\mathbf{v}_{n} \rightarrow \mathbf{v}$ in $\mathcal{C}_{\text {loc }}\left(\mathbb{R}^{N}\right)$ and in $H_{\text {loc }}^{1}\left(\mathbb{R}^{N}\right)$, and by Lemma 4.7 for any $r \in\left[\bar{r}+1, \tilde{r} / r_{n}\right)$

$$
\begin{aligned}
0 & \leq \frac{E(\mathbf{v}, 0, r)+H(\mathbf{v}, 0, r)}{r^{2}}=\lim _{n \rightarrow \infty} \frac{E\left(\mathbf{v}_{n}, 0, r\right)+H\left(\mathbf{v}_{n}, 0, r\right)}{r^{2}} \\
& \leq \lim _{n \rightarrow \infty} \sup _{r \in\left[\tilde{r}_{n}, \tilde{r} / r_{n}\right]} r_{n}^{2} \frac{E\left(\mathbf{v}_{n}, 0, \tilde{r} / r_{n}\right)+H\left(\mathbf{v}_{n}, 0, \tilde{r} / r_{n}\right)}{\tilde{r}^{2}} e^{\tilde{C}\left(\tilde{r}-r_{n} r\right)+\varphi_{n}(r)-\varphi_{n}\left(\tilde{r} / r_{n}\right)} \\
& \leq \lim _{n \rightarrow \infty} C r_{n}^{2} \frac{E\left(\mathbf{v}_{n}, 0, \tilde{r} / r_{n}\right)+H\left(\mathbf{v}_{n}, 0, \tilde{r} / r_{n}\right)}{\tilde{r}^{2}}=\lim _{n \rightarrow \infty} C \eta^{2}\left(x_{n}\right) \frac{E\left(\mathbf{u}_{\beta_{n}}, x_{n}, \tilde{r}\right)+H\left(\mathbf{u}_{\beta_{n}}, x_{n}, \tilde{r}\right)}{L_{n}^{2} \tilde{r}^{2}},
\end{aligned}
$$

where we used the identities 4.2 and the uniform boundedness of $\left\{\varphi_{n}\right\}$, see Remark 4.6 Since both $E\left(\mathbf{u}_{\beta_{n}}, x_{n}, \tilde{r}\right)$ and $H\left(\mathbf{u}_{\beta_{n}}, x_{n}, \tilde{r}\right)$ are also uniformly bounded (for the boundedness of $E\left(\mathbf{u}_{\beta_{n}}, x_{n}, \tilde{r}\right)$, it is possible to proceed as in points (5) and (6) of Lemma 2.1], while $L_{n} \rightarrow+\infty$, the last limit tends to 0 . As a consequence $\mathbf{v} \equiv 0$ in $B_{r}$, in contradiction with Lemma 4.5 .

Summing up, we have shown that if $L_{n} \rightarrow+\infty$ then necessarily

$$
\bar{r}_{n} \rightarrow \infty \quad \text { while } \quad r_{n} \bar{r}_{n} \rightarrow 0 \quad \text { as } n \rightarrow \infty \text {. }
$$

It remains to prove that also in this case we reach a contradiction with Lemma 4.5. To this end, let us introduce $J_{n}(r):=r^{-4} J_{1, n}(r) \cdot J_{2, n}(r)$, where

$$
\begin{aligned}
& J_{1, n}(r):=\int_{B_{r}}\left(\left|\nabla v_{1, n}\right|^{2}+M_{n} a_{12} v_{1, n}^{2} v_{2, n}^{2}-v_{1, n} f_{1, n}\left(x, v_{1, n}\right)\right)|x|^{2-N} \\
& J_{2, n}(r):=\int_{B_{r}}\left(\left|\nabla v_{2, n}\right|^{2}+M_{n} a_{12} v_{1, n}^{2} v_{2, n}^{2}-v_{2, n} f_{2, n}\left(x, v_{2, n}\right)\right)|x|^{2-N} .
\end{aligned}
$$

A crucial step in the proof of Theorem 1.3 is the validity of the Alt-Caffarelli-Friedman monotonicity formula of Subsection 3.2 for $J_{n}$, uniformly in $n$.

Lemma 4.9. There exists $C>0$ independent of $n$ such that $J_{1, n}(r) \geq C$ and $J_{2, n}(r) \geq C$ for every $r \in\left[2, \bar{r}_{n} / 3\right]$, and

$$
r \mapsto J_{n}(r) e^{-C M_{n}^{-1 / 4} r^{-1 / 2}+C r_{n}^{2} r^{2}} \quad \text { is monotone non-decreasing for } r \in\left[2, \bar{r}_{n} / 3\right] .
$$

The proof consists in verifying that the assumptions $(h 0)-(h 3)$ of Theorem 3.14 are satisfied in the range $\left[2, \bar{r}_{n} / 3\right]$, with constants uniform in $n$. In doing this, we shall strongly use the fact that $r \in\left(0, \bar{r}_{n}\right]$, the range where the function $\mathbf{v}_{n}$ has linear behaviour. Since the proof is quite long and a little bit technical, we postpone it in Subsection 4.1, and now we proceed with the conclusion of the proof of Theorem 1.3 .

Conclusion of the proof of Theorem 1.3. We aim at proving the validity of the following chain of inequalities, connecting the Alt-Caffarelli-Friedman monotonicity formula of Lemma 4.9 to the Almgren-type monotonicity formula of Lemma 4.7

$$
\begin{aligned}
C \leq J_{n}(2) & \leq C J_{n}\left(\frac{\bar{r}_{n}}{3}\right) \leq C\left(\frac{E\left(\mathbf{v}_{n}, 0, \bar{r}_{n}\right)+H\left(\mathbf{v}_{n}, 0, \bar{r}_{n}\right)}{\bar{r}_{n}^{2}}+o_{n}(1)\right)^{2} \\
& \leq C\left(r_{n}^{2} \frac{E\left(\mathbf{v}_{n}, 0, \tilde{r} / r_{n}\right)+H\left(\mathbf{v}_{n}, 0, \tilde{r} / r_{n}\right)}{\tilde{r}^{2}}+o_{n}(1)\right)^{2}
\end{aligned}
$$

where $o_{n}(1) \rightarrow 0$ as $n \rightarrow \infty$. Once that this is proved, the conclusion easily follows: indeed, as in Lemma 4.8. the last quantity tends to 0 as $n \rightarrow+\infty$, in contradiction with the fact that $J_{n}(2) \geq C$. Hence we 
have to verify the validity of 4.7). The first two inequalities follow by Lemma 4.9 , the last one can be proved as in Lemma 4.8, and therefore we have only to check that

$$
J_{n}\left(\frac{\bar{r}_{n}}{3}\right) \leq C\left(\frac{E\left(\mathbf{v}_{n}, 0, \bar{r}_{n}\right)+H\left(\mathbf{v}_{n}, 0, \bar{r}_{n}\right)}{\bar{r}_{n}^{2}}+o_{n}(1)\right)^{2} .
$$

We emphasize that, recalling the definition of $J_{n}(r)$ and $E\left(\mathbf{v}_{n}, 0, r\right)$, this can be considered an inequality relating the geometric mean with the arithmetic mean of suitable energy functionals of $\mathbf{v}_{n}$ in $B_{r}$. By definition

$$
\begin{aligned}
J_{n}\left(\frac{\bar{r}_{n}}{3}\right)= & \left(\frac{9}{\bar{r}_{n}^{2}} \int_{B_{\bar{r}_{n} / 3}}\left(\left|\nabla v_{1, n}\right|^{2}+M_{n} a_{12} v_{1, n}^{2} v_{2, n}^{2}-v_{1, n} f_{1, n}\left(x, v_{1, n}\right)\right)|x|^{2-N}\right) \\
& \cdot\left(\frac{9}{\bar{r}_{n}^{2}} \int_{B_{\bar{r}_{n} / 3}}\left(\left|\nabla v_{2, n}\right|^{2}+M_{n} a_{12} v_{1, n}^{2} v_{2, n}^{2}-v_{2, n} f_{2, n}\left(x, v_{2, n}\right)\right)|x|^{2-N}\right) .
\end{aligned}
$$

We control both terms in the product on the right hand side in the same way, so here we consider only the first term. It holds

$$
\begin{aligned}
& \frac{1}{\bar{r}_{n}^{2}} \int_{B_{\bar{r}_{n} / 3}}\left(\left|\nabla v_{1, n}\right|^{2}+M_{n} a_{12} v_{1, n}^{2} v_{2, n}^{2}-v_{1, n} f_{1, n}\left(x, v_{1, n}\right)\right)|x|^{2-N} \\
& \quad \leq \frac{1}{\bar{r}_{n}^{2}} \int_{B_{\bar{r}_{n}}}\left(\left|\nabla v_{1, n}\right|^{2}+M_{n} a_{12} v_{1, n}^{2} v_{2, n}^{2}-v_{1, n} f_{1, n}\left(x, v_{1, n}\right)\right)|x|^{2-N}+\frac{1}{\bar{r}_{n}^{2}} \int_{B_{\bar{r}_{n}} \backslash B_{\bar{r}_{n} / 3}} \frac{v_{1, n} f_{1, n}\left(x, v_{1, n}\right)}{|x|^{N-2}}
\end{aligned}
$$

First, we claim that

$$
\lim _{n \rightarrow \infty}\left|\frac{1}{\bar{r}_{n}^{2}} \int_{B_{\bar{r}_{n}} \backslash B_{\bar{r}_{n} / 3}} \frac{v_{1, n} f_{1, n}\left(x, v_{1, n}\right)}{|x|^{N-2}}\right|=0 .
$$

Indeed by definition of $v_{i, n}$ and $f_{i, n}$, and by the assumption 1.5 , it results

$$
\begin{aligned}
\left|\frac{1}{\bar{r}_{n}^{2}} \int_{B_{\bar{r}_{n}} \backslash B_{\bar{r}_{n} / 3}} \frac{v_{1, n} f_{1, n}\left(x, v_{1, n}\right)}{|x|^{N-2}}\right| & \leq \frac{d r_{n}^{2}}{\bar{r}_{n}^{2}} \int_{B_{\bar{r}_{n}} \backslash B_{\bar{r}_{n} / 3}} \frac{v_{1, n}^{2}}{|x|^{N-2}} \\
& =\frac{d \eta^{2}\left(x_{n}\right)}{L_{n}^{2} \bar{r}_{n}^{2} r_{n}^{2}} \int_{B_{\bar{r}_{n} r_{n}}\left(x_{n}\right) \backslash B_{\bar{r}_{n} r_{n} / 3}\left(x_{n}\right)} \frac{u_{1, \beta_{n}}^{2}}{\left|x-x_{n}\right|^{N-2}} \\
& \leq \frac{C m^{2}}{L_{n}^{2} \bar{r}_{n}^{N} r_{n}^{N}} \int_{B_{\bar{r}_{n} r_{n}}\left(x_{n}\right)} 1 \leq \frac{C}{L_{n}^{2}} \rightarrow 0
\end{aligned}
$$

as $n \rightarrow \infty$, where we used the uniform boundedness of $\left\{\mathbf{u}_{n}\right\}$ and the fact that $L_{n} \rightarrow+\infty$.

Secondly, we claim that

$$
\frac{1}{\bar{r}_{n}^{2}} \int_{B_{\bar{r}_{n}}}\left(\left|\nabla v_{1, n}\right|^{2}+M_{n} a_{12} v_{1, n}^{2} v_{2, n}^{2}-v_{1, n} f_{1, n}\left(x, v_{1, n}\right)\right)|x|^{2-N} \leq C \frac{E\left(\mathbf{v}_{n}, 0, \bar{r}_{n}\right)+H\left(\mathbf{v}_{n}, 0, \bar{r}_{n}\right)}{\bar{r}_{n}^{2}}
$$

To prove it, let us test the equation for $v_{i, n}$ against $v_{i, n}|x|^{2-N}$ : integrating by parts as in the proof of Lemma 3.18, we deduce that

$$
\begin{aligned}
\int_{B_{\bar{r}_{n}}}\left(\left|\nabla v_{1, n}\right|^{2}+M_{n} a_{12} v_{1, n}^{2} v_{2, n}^{2}-v_{1, n} f_{1, n}\left(x, v_{1, n}\right)\right)|x|^{2-N} \\
\leq \frac{1}{\bar{r}_{n}^{N-2}} \int_{\partial B_{\bar{r}_{n}}} v_{1, n} \partial_{\nu} v_{1, n}+\frac{N-2}{2 \bar{r}_{n}^{N-1}} \int_{\partial B_{\bar{r}_{n}}} v_{1, n}^{2} \\
\quad=\frac{1}{\bar{r}_{n}^{N-2}} \int_{B_{\bar{r}_{n}}}\left(\left|\nabla v_{1, n}\right|^{2}+M_{n} a_{12} v_{1, n}^{2} v_{2, n}^{2}-v_{1, n} f_{1, n}\left(x, v_{1, n}\right)\right)+\frac{N-2}{2 \bar{r}_{n}^{N-1}} \int_{\partial B_{\bar{r}_{n}}} v_{1, n}^{2} .
\end{aligned}
$$


For every $i=1, \ldots, k$ and for every $n$

$$
\begin{aligned}
& \frac{1}{\bar{r}_{n}^{N-2}} \int_{B_{\bar{r}_{n}}}\left(\left|\nabla v_{i, n}\right|^{2}+M_{n} a_{12} v_{i, n}^{2} \sum_{j \neq i} v_{j, n}^{2}-v_{i, n} f_{i, n}\left(x, v_{i, n}\right)\right)+\frac{N-2}{2 \bar{r}_{n}^{N-1}} \int_{\partial B_{\bar{r}_{n}}} v_{i, n}^{2} \\
& \geq \frac{\eta^{2}\left(x_{n}\right)}{L_{n}^{2} r_{n}^{2}}\left(\frac{1}{\left(\bar{r}_{n} r_{n}\right)^{N-2}} \int_{B_{\bar{r}_{n} r_{n}}\left(x_{n}\right)}\left|\nabla u_{i, \beta_{n}}\right|^{2}-\frac{d\left(\bar{r}_{n} r_{n}\right)^{2}}{\left(\bar{r}_{n} r_{n}\right)^{N}} \int_{B_{\bar{r}_{n} r_{n}}\left(x_{n}\right)} u_{i, \beta_{n}}^{2}\right) \\
& +\frac{\eta^{2}\left(x_{n}\right)}{L_{n}^{2} r_{n}^{2}} \frac{N-2}{2\left(\bar{r}_{n} r_{n}\right)^{N-1}} \int_{\partial B_{\bar{r}_{n} r_{n}}} u_{i, \beta_{n}}^{2} \\
& \geq \frac{\eta^{2}\left(x_{n}\right)}{L_{n}^{2} r_{n}^{2}} \frac{1}{\left(\bar{r}_{n} r_{n}\right)^{N-2}}\left(1-\frac{d\left(\bar{r}_{n} r_{n}\right)^{2}}{N-1}\right) \int_{B_{\bar{r}_{n} r_{n}}\left(x_{n}\right)}\left|\nabla u_{i, \beta_{n}}\right|^{2} \\
& +\frac{\eta^{2}\left(x_{n}\right)}{L_{n}^{2} r_{n}^{2}}\left(\frac{N-2}{2}-\frac{d\left(\bar{r}_{n} r_{n}\right)^{2}}{N-1}\right) \frac{1}{\left(\bar{r}_{n} r_{n}\right)^{N-1}} \int_{\partial B_{\bar{r}_{n} r_{n}}\left(x_{n}\right)} u_{i, \beta_{n}}^{2} \geq 0,
\end{aligned}
$$

where we used the fact that $\bar{r}_{n} r_{n} \rightarrow 0$ and the Poincaré inequality (Lemma 3.2. Coming back to 4.11, we deduce that

$$
\begin{aligned}
& \int_{B_{\bar{r}_{n}}}\left(\left|\nabla v_{1, n}\right|^{2}+M_{n} a_{12} v_{1, n}^{2} v_{2, n}^{2}-v_{1, n} f_{1, n}\left(x, v_{1, n}\right)\right)|x|^{2-N} \\
& \leq \sum_{i=1}^{k}\left[\frac{1}{\bar{r}_{n}^{N-2}} \int_{B_{\bar{r}_{n}}}\left(\left|\nabla v_{i, n}\right|^{2}+M_{n} a_{12} v_{i, n}^{2} \sum_{j \neq i} v_{j, n}^{2}-v_{i, n} f_{i, n}\left(x, v_{i, n}\right)\right)+\frac{N-2}{2 \bar{r}_{n}^{N-1}} \int_{\partial B_{\bar{r}_{n}}} v_{i, n}^{2}\right] \\
& =E\left(\mathbf{v}_{n}, 0, \bar{r}_{n}\right)+\frac{N-2}{2} H\left(\mathbf{v}_{n}, 0, \bar{r}_{n}\right) \leq C\left(E\left(\mathbf{v}_{n}, 0, \bar{r}_{n}\right)+H\left(\mathbf{v}_{n}, 0, \bar{r}_{n}\right)\right) \text {. }
\end{aligned}
$$

Multiplying the first and the last term by $\bar{r}_{n}^{-2}$, the claim 4.10 follows.

At this point it is sufficient to observe that claims 4.9) and 4.10 imply that 4.8 holds, which completes the proof.

4.1. Proof of Lemma 4.9. We will often use the fact that the function $N\left(\mathbf{v}_{n}, 0, r\right)$ can be controlled from below and from above by positive constants in the range $\left[2, \bar{r}_{n}\right]$.

Lemma 4.10. There exists $\sigma \in(0,1)$ such that $\sigma \leq N\left(\mathbf{v}_{n}, 0, r\right) \leq 1$ for every $r \in\left[2, \bar{r}_{n}\right]$, for every $n$. As a consequence

$$
\begin{aligned}
& r \mapsto \frac{H\left(\mathbf{v}_{n}, 0, r\right)}{r^{2}} \quad \text { is monotone non-increasing for } r \in\left[2, \bar{r}_{n}\right] \\
& r \mapsto \frac{H\left(\mathbf{v}_{n}, 0, r\right)}{r^{2 \sigma}} \quad \text { is monotone non-decreasing for } r \in\left[2, \bar{r}_{n}\right] .
\end{aligned}
$$

Proof. By the Almgren monotonicity formula

$$
\left(N\left(\mathbf{v}_{n}, 0, r\right)+1\right) e^{\tilde{C} r_{n} r} \leq\left(N\left(\mathbf{v}_{n}, 0, \bar{r}_{n}\right)+1\right) e^{\tilde{C} r_{n} \bar{r}_{n}}=2-r_{n} \bar{r}_{n}
$$

for every $r \in\left[0, \bar{r}_{n}\right]$. This gives the desired upper bound on $N$. For the lower bound, using again the Almgren monotonicity formula we have

$$
\left(N\left(\mathbf{v}_{n}, 0, r\right)+1\right) e^{\tilde{C} r_{n} r} \geq\left(N\left(\mathbf{v}_{n}, 0,2\right)+1\right) e^{\tilde{C} r_{n} 2}
$$

for every $r \in\left[0, \bar{r}_{n}\right]$, which readily implies

$$
N\left(\mathbf{v}_{n}, 0, r\right) \geq\left(N\left(\mathbf{v}_{n}, 0,2\right)+1\right) e^{-\tilde{C} r_{n} r}-1
$$

Now, as by Proposition 2.12 and Lemma 4.5 both $v_{1, n}$ and $v_{2, n}$ are non-trivial and non-constant in $B_{2}$, we have

$$
\int_{B_{2}}\left(\sum_{i}\left|\nabla v_{i, n}\right|^{2}+2 M_{n} \sum_{i<j} a_{i j} v_{i, n}^{2} v_{j, n}^{2}\right) \geq C>0
$$


Since $f_{i, n} \rightarrow 0$ uniformly in $\Omega_{n}$ (see point (1) of Lemma 2.1), we deduce that $E\left(\mathbf{v}_{n}, 0,2\right) \geq C>0$, and by uniform convergence $\mathbf{v}_{n} \rightarrow \mathbf{v}$ we infer that $N\left(\mathbf{v}_{n}, 0,2\right) \geq C>0$ independently of $n$. Since $r_{n} r \leq r_{n} \bar{r}_{n} \rightarrow 0$ as $n \rightarrow \infty$ for every $r \leq \bar{r}_{n}$, coming back to the estimate 4.12 we conclude that there exists $C>0$ such that

$$
N\left(\mathbf{v}_{n}, 0, r\right) \geq(1+C) e^{-\tilde{C} r_{n} r}-1 \geq \sigma>0 \quad \text { for every } r \in\left[2, \bar{r}_{n}\right],
$$

for every $n$ sufficiently large. The second part of the thesis is now a direct consequence of Lemma 3.6 $\bar{r}_{n}$.

In the next lemma we make rigorous the concept that $\mathbf{v}_{n}$ behaves in a linear way up to the threshold

Lemma 4.11. Let $\left(\rho_{n}\right)$ be any sequence such that $\rho_{n} \rightarrow \infty$ and $\rho_{n} \leq \bar{r}_{n} / 3$. Then there exist $\gamma>0$ and $1 \leq h<l \leq k$ such that, up to a subsequence, the blow-down sequence

$$
\tilde{v}_{i, n}(x):=\frac{v_{i, n}\left(\rho_{n} x\right)}{\sqrt{H\left(\mathbf{v}_{n}, 0, \rho_{n}\right)}}
$$

converges in $H^{1}\left(B_{1}\right) \cap \mathcal{C}\left(\overline{B_{1}}\right)$, up to a rotation, to the function $\tilde{\mathbf{v}}$ defined by

$$
\tilde{v}_{h}(x)=\gamma x_{1}^{+} \quad \tilde{v}_{l}(x)=\gamma x_{1}^{-} \quad \tilde{v}_{j}(x)=0 \quad \text { for every } j \neq h, l .
$$

Proof. We start with the observation that each $\tilde{\mathbf{v}}_{n}$ solves the system

$$
-\Delta \tilde{v}_{i, n}=\frac{\rho_{n}^{2}}{\sqrt{H\left(\mathbf{v}_{n}, 0, \rho_{n}\right)}} f_{i, n}\left(\rho_{n}, v_{i, n}\left(\rho_{n} x\right)\right)-\rho_{n}^{2} H\left(\mathbf{v}_{n}, 0, \rho_{n}\right) M_{n} \tilde{v}_{i, n} \sum_{j \neq i} a_{i j} \tilde{v}_{j, n}^{2}
$$

in a set $\tilde{\Omega}_{n} \supset B_{3}$ (this follows directly by the fact that $\Omega_{n} \supset B_{1 / r_{n}}$ ). Since $\rho_{n} \rightarrow+\infty, H\left(\mathbf{v}_{n}, 0, \rho_{n}\right) \geq C$ (by Lemma 4.5 and $M_{n} \geq C>0$, we infer that the new competition parameter $\rho_{n}^{2} H\left(\mathbf{v}_{n}, 0, \rho_{n}\right) M_{n} \rightarrow$ $+\infty$. Furthermore, recalling assumption (1.5) and the definition of $f_{i, n}$, we have

$$
-\Delta \tilde{v}_{i, n} \leq \frac{\rho_{n}^{2}}{\sqrt{H\left(\mathbf{v}_{n}, 0, \rho_{n}\right)}} f_{i, n}\left(\rho_{n}, v_{i, n}\left(\rho_{n} x\right)\right) \leq d\left(\rho_{n} r_{n}\right)^{2} \tilde{v}_{i, n}
$$

in $B_{3}$. We wish to deduce that $\left\{\tilde{\mathbf{v}}_{n}\right\}$ is uniformly bounded in $B_{2}$. If $\left\{\mathbf{v}_{n}\right\}$ is uniformly bounded in $H^{1}\left(B_{3}\right)$ and the coefficients on the right hand side are uniformly bounded, this follows by a classical Brezis-Kato argument. The boundedness of the coefficients is given by $\rho_{n} r_{n} \leq \bar{r}_{n} r_{n} \rightarrow 0$ as $n \rightarrow \infty$, as shown in Lemma 4.2 . Thus it remains to show that $\left\{\tilde{\mathbf{v}}_{n}\right\}$ is uniformly bounded in $H^{1}\left(B_{3}\right)$. By Lemma 4.10

$$
N\left(\tilde{\mathbf{v}}_{n}, 0, \rho\right) \leq N\left(\mathbf{v}_{n}, 0, \rho \rho_{n}\right) \leq 1
$$

for every $0 \leq \rho \leq 3$, so that by Lemma 3.6

$$
H\left(\tilde{\mathbf{v}}_{n}, 0, \rho\right)=\frac{H\left(\mathbf{v}_{n}, 0, \rho_{n} \rho\right)}{H\left(\mathbf{v}_{n}, 0, \rho_{n}\right)} \leq \rho^{2}
$$

for every $1 \leq \rho \leq 3$. Therefore

$$
E\left(\tilde{\mathbf{v}}_{n}, 0,3\right)=N\left(\tilde{\mathbf{v}}_{n}, 0,3\right) H\left(\tilde{\mathbf{v}}_{n}, 0,3\right) \leq 9 .
$$

It is easy to check that this gives the desired upper bound: indeed thanks to the Poincarè inequality in Lemma 3.2 we have

$$
\begin{aligned}
E\left(\tilde{\mathbf{v}}_{n}, 0,3\right) & \geq \frac{1}{3^{N-2}} \int_{\partial B_{3}} \sum_{i}\left|\nabla \tilde{v}_{i, n}\right|^{2}-\frac{\left(\rho_{n} r_{n}\right)^{2}}{3^{N}} \int_{B_{r}} \sum_{i} \tilde{v}_{i, n}^{2} \\
& \geq \frac{1}{3^{N-2}}\left(1-\left(\rho_{n} r_{n}\right)^{2}\right) \int_{B_{3}} \sum_{i}\left|\nabla \tilde{v}_{i, n}\right|^{2}-\left(\rho_{n} r_{n}\right)^{2} H\left(\tilde{\mathbf{v}}_{n}, 0,3\right) \\
& \geq C \int_{B_{3}} \sum_{i}\left|\nabla \tilde{v}_{i, n}\right|^{2}-o_{n}(1),
\end{aligned}
$$


with $o_{n}(1) \rightarrow 0$ as $n \rightarrow \infty$. Coming back to 4.13 , we deduce that the sequence $\left\{\tilde{\mathbf{v}}_{n}\right\}$ is bounded in $L^{\infty}\left(B_{2}\right)$, and in light of the local version of the main results in [21], see [28], we conclude that up to a subsequence $\tilde{\mathbf{v}}_{n} \rightarrow \tilde{\mathbf{v}}$ in $\mathcal{C}\left(B_{3 / 2}\right)$ and in $H^{1}\left(B_{3 / 2}\right)$ as $n \rightarrow \infty$. Since

$$
\left|\frac{\rho_{n}^{2}}{\sqrt{H\left(\mathbf{v}_{n}, 0, \rho_{n}\right)}} f_{i, n}\left(\rho_{n}, v_{i, n}\left(\rho_{n} x\right)\right)\right| \leq\left(\rho_{n} r_{n}\right)^{2}\left\|\tilde{v}_{i, n}\right\|_{L^{\infty}\left(B_{2}\right)} \rightarrow 0
$$

as $n \rightarrow \infty$, by Proposition 3.12 the limiting profile $\tilde{\mathbf{v}}$ belongs to the class $\mathcal{G}\left(B_{3 / 2}\right)$, with

$$
\Delta \tilde{v}_{i}=0 \quad \text { in }\left\{\tilde{v}_{i}>0\right\} .
$$

Moreover, $0 \in\{\tilde{\mathbf{v}}=0\}$, the free-boundary of $\tilde{\mathbf{v}}$, since the sequence $\left(\mathbf{v}_{n}(0)\right)$ is bounded in 0 while by Lemma 4.10

By Proposition 3.11 we conclude that

$$
H\left(\mathbf{v}_{n}, 0, \rho_{n}\right) \geq \frac{H\left(\mathbf{v}_{n}, 0,2\right)}{4^{\sigma}} r^{2 \sigma} \rightarrow+\infty \quad \text { as } n \rightarrow \infty .
$$

$$
1 \leq N\left(\tilde{\mathbf{v}}, 0,0^{+}\right) \leq N(\tilde{\mathbf{v}}, 0, r) \leq 1
$$

that is $N(\tilde{\mathbf{v}}, 0, r)=1$, for every $r \in(0,3 / 2]$, and hence $\tilde{\mathbf{v}}$ is homogeneous of degree 1 . Moreover, thanks to Theorem 3.13 the occurrence of self-segregation phenomena is not allowed, so that up to a rotation there exists $h, j \in\{1, \ldots, k\}$ such that

$$
\tilde{v}_{h}(x)=\gamma x_{1}^{+} \quad \tilde{v}_{l}(x)=\gamma x_{1}^{-} \quad \tilde{v}_{j}(x)=0 \quad \text { for every } j \neq h, l,
$$

where $\gamma>0$ is uniquely determined by the normalization condition $H(\tilde{\mathbf{v}}, 0,1)=1$.

Remark 4.12. The fact that $N\left(\mathbf{v}_{n}, 0, r\right) \leq 1$ below the threshold $\bar{r}_{n}$ implies that suitable scaled sequences $\left\{\tilde{\mathbf{v}}_{n}\right\}$ are converging to explicit segregated profile of linear type, as in the thesis of Lemma 4.11. If we exceed the threshold $\bar{r}_{n}$ in general $N\left(\mathbf{v}_{n}, 0, r\right)>1$, and the characterization of the possible blow-up limits becomes more involved and remains still an open problem.

The next step consists in showing that, up to the scale $\bar{r}_{n}$, there is a balance between $v_{1, n}$ and $v_{2, n}$, while the other components are of smaller order. Before, we need a technical result.

Definition 4.13. We denote as $\Sigma_{2, k}$ the subset of $\mathbb{R}^{k}$ of points with at most two non-trivial components, that is

$$
\Sigma_{2, k}:=\left\{\mathbf{x} \in \mathbb{R}^{k}: \exists i, j \in\{1, \ldots, k\} \text { such that } x_{h}=0 \forall h \neq i, j\right\} .
$$

For every $\mathbf{x} \in \mathbb{R}^{k}$ and $g \in \mathcal{C}\left([0,1] ; \mathbb{R}^{k}\right)$, we let

$$
\operatorname{dist}\left(\mathbf{x}, \Sigma_{2, k}\right)=\inf _{\mathbf{y} \in \Sigma_{2, k}}\|\mathbf{x}-\mathbf{y}\|, \quad \operatorname{dist}\left(g([0,1]), \Sigma_{2, k}\right)=\sup _{x \in[0,1]} \operatorname{dist}\left(f(x), \Sigma_{2, k}\right) .
$$

Lemma 4.14. Let $\varepsilon \in(0,1)$ be a fixed number and let $g_{n} \in \mathcal{C}\left([0,1], \mathbb{R}^{k}\right)$ be a sequence of continuous functions such that

$$
g_{n}([0,1]) \subset E_{\varepsilon}:=\left\{\mathbf{x}=\left(x_{1}, \ldots, x_{k}\right) \in \mathbb{R}^{k}: x_{i} \geq 0, x_{i} \leq 1-\varepsilon, \sum_{i=1}^{k} x_{i}=1\right\} .
$$

If the limit

$$
\lim _{n \rightarrow \infty} \operatorname{dist}\left(g_{n}([0,1]), \Sigma_{2, k}\right)=0
$$

holds true, then (up to a subsequence) there exist $i \neq j$ such that for $n$ sufficiently large

$$
\frac{\varepsilon}{2}<g_{i, n}(x), g_{j, n}(x)<1-\frac{\varepsilon}{2} \quad \text { and } \quad g_{h, n} \rightarrow 0 \quad \text { uniformly in }[0,1] \text { for } h \neq i, j .
$$

Proof. The set $E_{\varepsilon} \cap \Sigma_{2, k}$ is made of $k(k-1) / 2$ connected components, given by the reflections of the set

$$
A=\left\{x_{1}+x_{2}=1, \varepsilon \leq x_{1} \leq 1-\varepsilon, \varepsilon \leq x_{2} \leq 1-\varepsilon, x_{i}=0 \forall i \geq 3\right\} .
$$

For the assumptions we evince that for any $\delta>0$, there exists $\bar{n}$ sufficiently large such that

$$
g_{n}([0,1]) \subset\left(E_{\varepsilon} \cap \Sigma_{2, k}\right)+B_{\delta} \quad \text { for all } n \geq \bar{n} .
$$


Evidently, for $\delta$ small enough, the set $\left(E_{\varepsilon} \cap \Sigma_{2, k}\right)+B_{\delta}$ is again made of $k(k-1) / 2$ disjoint components. It follows then that for a suitable subsequence there exists a connected component of $E_{\varepsilon} \cap \Sigma_{2, k}$, say the set $A$, such that

$$
\lim _{n \rightarrow \infty} \operatorname{dist}\left(g_{n}([0,1]), A\right)=0 .
$$

The thesis is then an immediate consequence.

Lemma 4.15. There exists $\lambda>0$ independent of $n$ such that

$$
\frac{1}{\lambda} \leq \frac{\int_{\partial B_{r}} v_{1, n}^{2}}{\int_{\partial B_{r}} v_{2, n}^{2}} \leq \lambda
$$

for every $2 \leq r \leq \bar{r}_{n} / 3$, while on the contrary for $j=3, \ldots, k$ we have

$$
\sup _{r \in\left[2, \bar{r}_{n} / 3\right]} \frac{\int_{\partial B_{r}} v_{j, n}^{2}}{\int_{\partial B_{r}} v_{1, n}^{2}} \rightarrow 0 \quad \text { as } n \rightarrow \infty .
$$

Proof. The proof is based on an application of Lemma 4.14 more precisely, given the sequence $\left\{\mathbf{v}_{n}\right\}$, let us introduce the family of auxiliary functions

$$
g_{i, n}(\rho):= \begin{cases}\frac{1}{H\left(\mathbf{v}_{n}, 0, \rho \bar{r}_{n} / 3\right)} \frac{1}{\left(\rho \bar{r}_{n} / 3\right)^{N-1}} \int_{\partial B_{\rho \bar{r}_{n} / 3}} v_{i, n}^{2} & \text { for } \frac{6}{\bar{r}_{n}} \leq \rho \leq 1 \\ \frac{1}{H\left(\mathbf{v}_{n}, 0,2\right)} \frac{1}{2^{N-1}} \int_{\partial B_{2}} v_{i, n}^{2} & \text { for } 0 \leq \rho \leq \frac{6}{\bar{r}_{n}} .\end{cases}
$$

the thesis follows once we have shown that $\left\{g_{n}\right\}$ satisfies the assumptions of Lemma 4.14 . By construction, we have that each $g_{i, n}$ is continuous, $g_{i, n} \geq 0$, and $\sum_{i=1}^{k} g_{i, n}(x)=1$ for all $x \in[0,1]$.

Step 1) There exists $\varepsilon \in(0,1)$ such that $g_{i, n}(x) \leq 1-\varepsilon$ for all $x \in[0,1]$, independently of $n$. By contradiction, let us assume that is there exist an index $i$ and a sequence $s_{n} \in[0,1]$ such that

$$
g_{i, n}\left(s_{n}\right) \rightarrow 1 \quad \text { and } \quad g_{j, n}\left(s_{n}\right) \rightarrow 0 \quad \forall j \neq i .
$$

The local uniform convergence $\mathbf{v}_{n} \rightarrow \mathbf{v}$ and Lemma 4.5 imply that necessarily $s_{n} \bar{r}_{n} \rightarrow+\infty$ as $n \rightarrow \infty$. Let us introduce a new sequence

$$
\tilde{v}_{i, n}(x):=\frac{v_{i, n}\left(s_{n} \bar{r}_{n} x / 3\right)}{\sqrt{H\left(\mathbf{v}_{n}, 0, s_{n} \bar{r}_{n} / 3\right)}} .
$$

We are in a position to apply Lemma 4.11 (with $\rho_{n}:=s_{n} \bar{r}_{n} / 3$ ) and to conclude that the uniform limit of $\left\{\tilde{\mathbf{v}}_{n}\right\}$ contains at least two non trivial components, in contradiction with (4.14).

Step 2) $\lim _{n \rightarrow \infty} \operatorname{dist}\left(g_{n}([0,1]), \Sigma_{2, k}\right)=0$. As before, let us assume by contradiction, that there exist $\varepsilon>0$, three distinct indices $i, j, k$, and a sequence $s_{n} \in(0,1)$ such that up to a subsequence

$$
g_{i, n}\left(s_{n}\right) \geq \varepsilon, \quad g_{j, n}\left(s_{n}\right) \geq \varepsilon, \quad g_{k, n}\left(s_{n}\right) \geq \varepsilon
$$

for any $n$ sufficiently large, and let us introduce again the sequence

$$
\tilde{v}_{i, n}(x):=\frac{v_{i, n}\left(s_{n} \bar{r}_{n} x / 3\right)}{\sqrt{H\left(\mathbf{v}_{n}, 0, s_{n} \bar{r}_{n} / 3\right)}} .
$$

As a result of Lemma 4.11 the uniform limit of $\left\{\tilde{\mathbf{v}}_{n}\right\}$ contains at most two non trivial components, a contradiction.

Remark 4.16. We point out that such proof rests upon Lemma 4.11, which reflects the linear behaviour of $\mathbf{v}_{n}$ in $B_{r}$ for $r \in\left(0, \bar{r}_{n}\right]$. We do not expect that in a superlinear range the same result holds.

Lemmas 4.5 and 4.15 imply that assumption $(h 2)$ in Theorem 3.14 is satisfied with constants $\mu, \lambda>0$ independent of $n$ in the interval $r \in\left(2, \bar{r}_{n} / 3\right)$. We now consider assumptions $(h 0)$ and $(h 3)$. 
Lemma 4.17. Provided $n$ is sufficiently large, there holds

$$
\left|f_{i, n}\left(x, v_{i, n}\right)\right| \leq d r_{n}^{2} v_{i, n} \quad \text { in } \Omega_{n} \quad \text { with } \quad \frac{d r_{n}^{2} \bar{r}_{n}^{2}}{9}<\left(\frac{N-2}{2}\right)^{2}
$$

for $i=1,2$. We recall that $\Omega_{n}$ is the domain of definition of $\mathbf{v}_{n}$.

Proof. It is an easy consequence of the definition of $f_{i, n}$, and of the fact that $r_{n}^{2} \bar{r}_{n}^{2} \rightarrow 0$ as $n \rightarrow \infty$, see Lemma 4.2 .

At this point we can show that the quantities $\Lambda_{1, n}(r)$ and $\Lambda_{2, n}(r)$ are uniformly bounded from below by a positive constant up to the scale $\bar{r}_{n}$.

Lemma 4.18. There exists $C>0$ independent of $n$ such that

$$
\Lambda_{1, n}(r), \Lambda_{2, n}(r) \geq C \quad \text { for } r \in\left[2, \frac{\bar{r}_{n}}{3}\right]
$$

Proof. By contradiction, we assume that there exists $\rho_{n} \in\left[2, \bar{r}_{n} / 3\right]$ such that (without loss of generality) $\lim _{n} \Lambda_{1, n}\left(\rho_{n}\right) \leq 0$, that is

$$
\lim _{n \rightarrow \infty} \frac{\rho_{n}^{2} \int_{\partial B_{\rho_{n}}}\left|\nabla_{\theta} v_{1, n}\right|^{2}+M_{n} a_{12} v_{1, n}^{2} v_{2, n}^{2}-v_{1, n} f_{1, n}\left(x, v_{1, n}\right)}{\int_{\partial B_{\rho_{n}}} v_{1, n}^{2}} \leq 0 .
$$

Either $\rho_{n} \rightarrow+\infty$ or $\left(\rho_{n}\right)$ is bounded. In the former case, we consider the scaled sequence

$$
\tilde{v}_{i, n}(x):=\frac{v_{i, n}\left(\rho_{n} x\right)}{\sqrt{H\left(\mathbf{v}_{n}, 0, \rho_{n}\right)}},
$$

which is well defined in $B_{3}$ since $\rho_{n} \leq \bar{r}_{n} / 3$. The asymptotic behaviour of this blow-down sequence is again contained in Lemma 4.11, from which we know that $\tilde{\mathbf{v}}_{n} \rightarrow \tilde{\mathbf{v}}$ such that (up to a rotation)

$$
\tilde{v}_{i}=\gamma x_{1}^{+}, \quad \tilde{v}_{j}=\gamma x_{1}^{-}, \quad \tilde{v}_{k}=0 \text { for every } k \neq i, j,
$$

for a suitable $\gamma>0$. Thanks to Lemma 4.15, it is necessary that $i=1$ and $j=2$. The knowledge of the limiting profile of the blow-down sequence $\left\{\tilde{\mathbf{v}}_{n}\right\}$ allows us to pass from the uniform convergence $\tilde{\mathbf{v}}_{n} \rightarrow \tilde{\mathbf{v}}$ to the $\mathcal{C}^{1, \alpha}$ convergence $\tilde{v}_{1, n} \rightarrow \tilde{v}_{1}$ far away from the free-boundary, and in particular in a set of type $\left\{\gamma x_{1}>2 \delta\right\}$; here $\delta>0$ is a sufficiently small quantity, such that $\partial B_{1} \cap\left\{\gamma x_{1}>2 \delta\right\} \neq \emptyset$. To prove this, we observe that by uniform convergence $\tilde{v}_{1, n} \geq C>0$ in $B_{2} \cap\left\{\gamma x_{1}>\delta\right\}$. Let $x_{0} \in B_{2} \cap\left\{\gamma x_{1}>2 \delta\right\}$, and let $\rho>0$ so small that for any such $x_{0}$ the ball $B_{\rho}\left(x_{0}\right)$ is contained in $\left\{\gamma x_{1}>\delta\right\}$. Lemma 2.2 applies to

$$
\left\{\begin{array}{l}
-\Delta \tilde{v}_{j, n} \leq\left(\rho_{n}^{2} r_{n}^{2}-C a_{1 j} H\left(\mathbf{v}_{n}, 0, \rho_{n}\right) \rho_{n}^{2} M_{n}\right) \tilde{v}_{j, n} \leq-C a_{1 j} H\left(\mathbf{v}_{n}, 0, \rho_{n}\right) \rho_{n}^{2} M_{n} \tilde{v}_{j, n} \\
\tilde{v}_{j, n} \geq 0 \\
\tilde{v}_{j, n} \leq C,
\end{array}\right.
$$

for every $j \neq 1$, implying that

$$
H\left(\mathbf{v}_{n}, 0, \rho_{n}\right) \rho_{n}^{2} M_{n} \tilde{v}_{j, n}\left(x_{0}\right) \leq C \quad \text { for every } x_{0} \in B_{2-\rho} \cap\left\{\gamma x_{1}>2 \delta\right\} .
$$

As a consequence, $\left\{\Delta v_{1, n}\right\}$ is uniformly bounded in $B_{2-\rho} \cap\left\{\gamma x_{1}>2 \delta\right\}$, which together with the uniform bound of $\left\{v_{1, n}\right\}$ in the same set provides uniform boundedness in any space $\mathcal{C}^{1, \alpha}\left(B_{2-\rho} \cap\left\{\gamma x_{1}>2 \delta\right\}\right)$. Therefore the convergence $\tilde{v}_{1, n} \rightarrow \tilde{v}_{1}$ takes place in $\mathcal{C}^{1, \alpha}\left(B_{2-\rho} \cap\left\{\gamma x_{1}>2 \delta\right\}\right)$ for any $0<\alpha<1$.

This finally permits to reach a contradiction: indeed we have

$$
\begin{aligned}
0 & \geq \lim _{n \rightarrow \infty} \frac{\rho_{n}^{2} \int_{\partial B_{\rho_{n}}}\left|\nabla_{\theta} v_{1, n}\right|^{2}+M_{n} a_{12} v_{1, n}^{2} v_{2, n}^{2}-v_{1, n} f_{1, n}\left(x, v_{1, n}\right)}{\int_{\partial B_{\rho_{n}}} v_{1, n}^{2}} \\
& \geq \lim _{n \rightarrow \infty} \frac{\int_{\partial B_{1}}\left|\nabla_{\theta} \tilde{v}_{1, n}\right|^{2}}{\int_{\partial B_{1}} \tilde{v}_{1, n}^{2}}-\lim _{n \rightarrow \infty} \rho_{n}^{2} \frac{\int_{\partial B_{\rho_{n}}} v_{1, n} f_{1, n}\left(x, v_{1, n}\right)}{\int_{\partial B_{\rho_{n}}} v_{1, n}^{2}} \geq C>0,
\end{aligned}
$$


where the last inequalities follow from the fact that by $\mathcal{C}^{1, \alpha}$ convergence

$$
\lim _{n \rightarrow \infty} \frac{\int_{\partial B_{1}}\left|\nabla_{\theta} \tilde{v}_{1, n}\right|^{2}}{\int_{\partial B_{1}} \tilde{v}_{1, n}^{2}} \geq \lim _{n \rightarrow \infty} \frac{\int_{\partial B_{1} \cap\left\{\gamma x_{1}>2 \delta\right\}}\left|\nabla_{\theta} \tilde{v}_{1, n}\right|^{2}}{\int_{\partial B_{1}} \tilde{v}_{1, n}^{2}}=\frac{\int_{\partial B_{1} \cap\left\{\gamma x_{1}>2 \delta\right\}}\left|\nabla_{\theta}\left(\gamma x_{1}\right)\right|^{2}}{\int_{\partial B_{1}}\left(\gamma x_{1}^{+}\right)^{2}}=C>0,
$$

while on the contrary in light of the definition of $f_{i, n}$ and on assumption 1.5

$$
\lim _{n \rightarrow \infty}\left|\rho_{n}^{2} \frac{\int_{\partial B_{\rho_{n}}} v_{1, n} f_{1, n}\left(x, v_{1, n}\right)}{\int_{\partial B_{\rho_{n}}} v_{1, n}^{2}}\right| \leq d \rho_{n}^{2} r_{n}^{2}=0 .
$$

At this point it remains to prove the result when $\rho_{n} \leq \bar{r}$. In such a situation the proof is much easier, as it is not necessary to introduce the scaling $\left\{\tilde{\mathbf{v}}_{n}\right\}$, but it is sufficient to argue on the original sequence $\left\{\mathbf{v}_{n}\right\}$. If $M_{n} \rightarrow+\infty$, then since $N\left(\mathbf{v}_{n}, 0, r\right) \leq 1$ for every $r \leq \bar{r}_{n}$ we have that up to a rotation $v_{1, n} \rightarrow v_{1}=\gamma x_{1}^{+}$, and the convergence takes place in $\mathcal{C}^{1, \alpha}$ in any compact subset of $\left\{\gamma x_{1}>\delta\right\}$. Then the conclusion follows exactly as before. If $\left(M_{n}\right)$ is bounded, then as observed in Proposition $2.12 \mathbf{v}_{n} \rightarrow \mathbf{v}$ in $\mathcal{C}_{\text {loc }}^{1, \alpha}\left(\mathbb{R}^{N}\right)$, and since both $v_{1}$ and $v_{2}$ are non-trivial (this follows from Lemma 4.5), by the strong maximum principle $v_{1}, v_{2}>0$ in $\mathbb{R}^{N}$. Since $\left(\rho_{n}\right)$ is also bounded, up to a subsequence $\rho_{n} \rightarrow \bar{\rho} \geq 2$. Recalling that $M_{n} \geq C>0$, we deduce that

$$
\frac{\bar{\rho}^{2} \int_{B_{\bar{\rho}}} M_{n} a_{12} v_{1, n}^{2} v_{2, n}^{2}}{\int_{B_{\bar{\rho}}} v_{1, n}^{2}} \geq C>0,
$$

which allows to obtain a contradiction.

It remains to show that also $J_{1, n}(r)$ and $J_{2, n}(r)$ are positive in the whole range $\left[2, \bar{r}_{n} / 3\right]$.

Lemma 4.19. There exists $C>0$ independent of $n$ such that

$$
J_{i, n}(r)>C \quad \text { for every } r \in\left[2, \frac{\bar{r}_{n}}{3}\right],
$$

for $i=1,2$.

Proof. First of all, there exists $\bar{C}>0$ such that $J_{i, n}(r) \geq \bar{C}$ for every $r \in[2,10]$ and $i=1,2$. This is a simple consequence of the $\mathcal{C}\left(B_{10}\right)$ and $H^{1}\left(B_{10}\right)$ convergence $v_{i, n} \rightarrow v_{i}$, with $v_{i} \not \equiv 0$ and not constant in $B_{10}$ for $i=1,2$, and of the fact that $f_{i, n}\left(x, v_{i, n}(x)\right) \rightarrow 0$ uniformly in $B_{10}$, see Lemma 2.1. Let now

$$
s_{n}:=\sup \left\{s \in\left(2, \bar{r}_{n} / 3\right): J_{i, n}(r)>\bar{C} / 10 \text { for every } r \in(2, s)\right\} .
$$

Note that $s_{n} \geq 10$ is well defined. In light of Lemmas 4.5 and 4.154 .18 , the assumptions of Theorem 3.14 are satisfied in the interval $\left(2, s_{n}\right)$, uniformly in $n$. As a consequence there exists $C>0$ such that

$$
r \mapsto \frac{J_{1, n}(r) J_{2, n}(r)}{r^{4}} \exp \left\{-C M_{n}^{-1 / 4} r^{-1 / 2}+C r_{n}^{2} r^{2}\right\}
$$

is monotone non-decreasing for $r \in\left(2, s_{n}\right)$. We claim that $s_{n}=\bar{r}_{n} / 3$ : indeed for every $r \in\left(2, s_{n}\right)$ it results

$$
J_{n}(r) \geq J_{n}(2) e^{-C M_{n}^{-1 / 4}+C r_{n}^{2}} e^{C M_{n}^{-1 / 4} s_{n}^{-1 / 2}-C r_{n}^{2} s_{n}^{2}} \geq \frac{\bar{C}}{10}>0
$$

at least for $n$ sufficiently large, which proves the claim.

Conclusion of the proof of Lemma 4.9. By Lemmas 4.5 4.19, the assumptions of Theorem 3.14 are satisfied by $\left(v_{1, n}, v_{2, n}\right)$ for $r \in\left[2, \bar{r}_{n} / 3\right]$, with constants $\mu, \lambda$ and $\varepsilon$ independent of $n$.

\section{The COMPLETELY SYMMETRIC INTERACTION}

This section is devoted to the study of the Lipschitz uniform continuity of the system (1.9p:

$$
\left\{\begin{array}{l}
-\Delta u_{i, \beta}=f_{i, \beta}\left(x, u_{i, \beta}\right)-\beta u_{i, \beta} \sum_{j \neq i} u_{j, \beta} \\
u_{i, \beta}>0
\end{array}\right.
$$

In Section 2, under rather general assumptions on the competition term, we established the asymptotic properties of two blow-up sequences $\left\{\mathbf{v}_{n}\right\}$ and $\left\{\overline{\mathbf{v}}_{n}\right\}$, which have been introduced starting from the assumption that a uniform bound on the Lipschitz norm of $\left\{\mathbf{u}_{\beta_{n}}\right\}$ does not exist. In what follows, 
we will show the such asymptotic properties bring us to a contradiction. We shall make use of the celebrated almost monotonicity formula of Caffarelli-Jerison-Kenig [5], which we recall here in its original formulation. For any given $u, v \in H^{1}$ functions, we let

$$
\Phi(r):=\left(\frac{1}{r^{2}} \int_{B_{r}} \frac{|\nabla u|^{2}}{|x|^{N-2}}\right)\left(\frac{1}{r^{2}} \int_{B_{r}} \frac{|\nabla v|^{2}}{|x|^{N-2}}\right) .
$$

Theorem 5.1 (Caffarelli-Jerison-Kenig almost monotonicity). Suppose $u$, $v$ are non-negative, continuous functions on the unit ball $B_{1}$. Suppose that $-\Delta u \leq 1$ and $-\Delta v \leq 1$ in the sense of distributions and that $u(x) v(x)=0$ for all $x \in B_{1}$. Then there exists a constant $C$ depending only on the dimension such that

$$
\Phi(r) \leq C\left(1+\int_{B_{r}} \frac{|\nabla u|^{2}}{|x|^{N-2}}+\int_{B_{r}} \frac{|\nabla v|^{2}}{|x|^{N-2}}\right)^{2}, \quad \text { for every } 0<r \leq 1 .
$$

Moreover, if $u$ and $v$ satisfy the same assumptions also in the ball $B_{2}$, then there exists a dimensional constant $C>0$ such that

$$
\Phi(r) \leq C\left(1+\int_{B_{2}} u^{2}+\int_{B_{2}} v^{2}\right)^{2}, \quad 0<r \leq 1 .
$$

One of the main consequences of the previous theorem is that the function $\Phi$ is bounded uniformly in $r$ whenever $u$ and $v$ are bounded in the ball $B_{2}$. In particular, in our setting we have the following.

Lemma 5.2. Let $\bar{r}>0$ be fixed. There exists a constant $C>0$ independent of $\beta$ such that for any $r \leq \bar{r} / 2$ and $x_{0}$ for which $B_{\bar{r}}\left(x_{0}\right) \subset \Omega$, the estimate

$$
\frac{1}{r^{N}} \int_{B_{r}\left(x_{0}\right)}\left|\nabla\left(u_{i, \beta}-u_{j, \beta}\right)^{+}\right|^{2} \cdot \frac{1}{r^{N}} \int_{B_{r}\left(x_{0}\right)}\left|\nabla\left(u_{i, \beta}-u_{j, \beta}\right)^{-}\right|^{2} \leq C
$$

holds for any $i \neq j$ and $\beta>0$.

Proof. For any two indices $i \neq j$, a straightforward computation shows that

$$
-\Delta\left(u_{i, \beta}-u_{j, \beta}\right)+\beta\left(u_{i, \beta}-u_{j, \beta}\right) \sum_{h \neq i, j} u_{h, \beta}=f_{i, \beta}\left(x, u_{i, \beta}\right)-f_{j, \beta}\left(x, u_{j, \beta}\right)
$$

where the right hand side is, by assumption, uniformly bounded in $L^{\infty}$. It follows that the positive and the negative part of $\left(u_{i, \beta}-u_{j, \beta}\right)$ fall under the assumptions of the Caffarelli-Jerison-Kenig monotonicity formula, and in particular

$$
\begin{aligned}
& \frac{1}{r^{N}} \int_{B_{r}\left(x_{0}\right)}\left|\nabla\left(u_{i, \beta}-u_{j, \beta}\right)^{+}\right|^{2} \cdot \frac{1}{r^{N}} \int_{B_{r}\left(x_{0}\right)}\left|\nabla\left(u_{i, \beta}-u_{j, \beta}\right)^{-}\right|^{2} \\
& \leq\left(\frac{1}{r^{2}} \int_{B_{r}\left(x_{0}\right)} \frac{\left|\nabla\left(u_{i, \beta}-u_{j, \beta}\right)^{+}\right|^{2}}{\left|x-x_{0}\right|^{N-2}}\right)\left(\frac{1}{r^{2}} \int_{B_{r}\left(x_{0}\right)} \frac{\left|\nabla\left(u_{i, \beta}-u_{j, \beta}\right)^{-}\right|^{2}}{\left|x-x_{0}\right|^{N-2}}\right) \\
& \quad \leq C\left\|f_{i, \beta}-f_{j, \beta}\right\|_{L^{\infty}}^{2}\left(1+\int_{B_{2}\left(x_{0}\right)} u_{i, \beta}^{2}+\int_{B_{2}\left(x_{0}\right)} u_{j, \beta}^{2}\right)^{2}
\end{aligned}
$$

where the last term is, by assumption, uniformly bounded in $\beta$.

Corollary 5.3. Any blow-up limit $\mathbf{v}$ is made of ordered functions, that is, for any pair $i \neq j$ either $v_{i} \leq v_{j}$ or $v_{j} \leq v_{i}$ in the whole $\mathbb{R}^{N}$.

Proof. Indeed, scaling properly the estimate (5.1), we obtain for every $r \in\left(0,1 / r_{n}\right)$ and $n$ large enough

$$
\frac{1}{r^{N}} \int_{B_{r}}\left|\nabla\left(v_{i, n}-v_{j, n}\right)^{+}\right|^{2} \cdot \frac{1}{r^{N}} \int_{B_{r}}\left|\nabla\left(v_{j, n}-v_{i, n}\right)^{-}\right|^{2} \leq \frac{\eta\left(x_{n}\right)^{4}}{L_{n}^{4}} C \rightarrow 0
$$

as $n \rightarrow \infty$. The conclusion follows by strong $H_{\text {loc }}^{1}\left(\mathbb{R}^{N}\right)$ convergence of the blow-up sequence and by the continuity of the blow-up limit.

We now recall a classical result, for which we refer to Lemma 2 in 4 . 
Lemma 5.4. Let $1<p<\infty$, and let $u \in L_{\mathrm{loc}}^{p}\left(\mathbb{R}^{N}\right)$ be a solution of

$$
-\Delta u \leq-|u|^{p-1} u \quad \text { in } \mathbb{R}^{N},
$$

in the sense of distributions. Then $u \leq 0$. In particular, if we assume $u$ to be non-negative, then $u \equiv 0$.

Proof of Theorem 1.4. We divided the proof in two steps.

Step 1) the case $\left(M_{n}\right)$ bounded. In this case by Proposition 2.12 the limiting function $\mathbf{v}$ is a non-negative, non-trivial, non-constant and sublinear solution of

$$
-\Delta v_{1}=-M_{\infty} v_{1} v_{2} \quad-\Delta v_{2}=-M_{\infty} v_{1} v_{2} \quad v_{j} \equiv 0 \quad \text { for every } j \neq 1,2,
$$

for some $M_{\infty}>0$. By Corollary 5.3 we evince that either $v_{1} \geq v_{2}$ in $\mathbb{R}^{N}$, or $v_{2} \geq v_{1}$ in $\mathbb{R}^{N}$. We shall show that in such situation $v_{1} \equiv v_{2} \equiv 0$ in $\mathbb{R}^{N}$, a contradiction. Without loss of generality, we suppose that $v_{1} \not \equiv 0$ and $v_{1} \geq v_{2}$. Thanks to Lemma 5.4

$$
-\Delta v_{2}=-M_{\infty} v_{1} v_{2} \leq-M_{\infty} v_{2}^{2} \quad \Longrightarrow \quad v_{2} \equiv 0 .
$$

But then $v_{1}$ is a non-constant positive harmonic function, in contradiction with the classical Liouville theorem.

Step 2) the case $M_{n} \rightarrow+\infty$. In such a situation, the segregation condition (Proposition 2.12, (2.8)) implies that all the component of the vector $\mathbf{v}$ are all trivial with the exception of $v_{1}$, which is then a subharmonic non-constant function. Letting

$$
\hat{v}_{1, n}:=v_{1, n}-\sum_{j \neq 1} v_{j, n}
$$

we immediately obtain that also $\hat{v}_{1, n} \rightarrow v_{1}$ locally uniformly in $\mathbb{R}^{N}$. But at the same time, a direct computation shows that

$$
-\Delta \hat{v}_{1, n} \geq f_{1, n}\left(x, v_{1, n}\right)-\sum_{j \neq 1} f_{j, n}\left(x, v_{j, n}\right) \quad \text { in } \Omega_{n}
$$

where the right hand side vanishes uniformly as $n \rightarrow+\infty$, implying that the function $v_{1}$ is also superharmonic. This again forces $v_{1}$ to be a non-constant positive harmonic function, in contradiction with the classical Liouville theorem.

Acknowledgements: we wish to thank Benedetta Noris for a careful reading of the first version of this paper. The authors are partially supported through the project ERC Advanced Grant 2013 n. 339958 "Complex Patterns for Strongly Interacting Dynamical Systems - COMPAT".

\section{REFERENCES}

[1] H. W. Alt, L. A. Caffarelli, and A. Friedman. Variational problems with two phases and their free boundaries. Trans. Amer. Math. Soc., 282(2):431-461, 1984.

[2] H. Berestycki, T.-C. Lin, J. Wei, and C. Zhao. On phase-separation models: asymptotics and qualitative properties. Arch. Ration. Mech. Anal., 208(1):163-200, 2013.

[3] H. Berestycki, S. Terracini, K. Wang, and J. Wei. On entire solutions of an elliptic system modeling phase separations. Adv. Math., 243:102-126, 2013.

[4] H. Brezis. Semilinear equations in $\mathbf{R}^{N}$ without condition at infinity. Appl. Math. Optim., 12(3):271-282, 1984.

[5] L. A. Caffarelli, D. Jerison, and C. E. Kenig. Some new monotonicity theorems with applications to free boundary problems. Ann. of Math. (2), 155(2):369-404, 2002.

[6] L. A. Caffarelli, A. L. Karakhanyan, and F.-H. Lin. The geometry of solutions to a segregation problem for nondivergence systems. J. Fixed Point Theory Appl., 5(2):319-351, 2009.

[7] L. A. Caffarelli and F.-H. Lin. Singularly perturbed elliptic systems and multi-valued harmonic functions with free boundaries. J. Amer. Math. Soc., 21(3):847-862, 2008.

[8] L. A. Caffarelli and J.-M. Roquejoffre. Uniform Hölder estimates in a class of elliptic systems and applications to singular limits in models for diffusion flames. Arch. Ration. Mech. Anal., 183(3):457-487, 2007.

[9] L. A. Caffarelli and S. Salsa. A geometric approach to free boundary problems, volume 68 of Graduate Studies in Mathematics. American Mathematical Society, Providence, RI, 2005.

[10] S.-M. Chang, C.-S. Lin, T.-C. Lin, and W.-W. Lin. Segregated nodal domains of two-dimensional multispecies BoseEinstein condensates. Phys. D, 196(3-4):341-361, 2004. 
[11] M. Conti, S. Terracini, and G. Verzini. A variational problem for the spacial segregation of reaction-diffusion systems. Indiana Univ. Math. J., 54(3):779-815, 2005.

[12] M. Conti, S. Terracini, and G. Verzini. Nehari's problem and competing species systems. Ann. Inst. H. Poincaré Anal. Non Linéaire, 19(6):871-888, 2002.

[13] M. Conti, S. Terracini, and G. Verzini. An optimal partition problem related to nonlinear eigenvalues. J. Funct. Anal., 198(1):160-196, 2003.

[14] M. Conti, S. Terracini, and G. Verzini. Asymptotic estimates for the spatial segregation of competitive systems. Adv. Math., 195(2):524-560, 2005.

[15] E. N. Dancer, K. Wang, and Z. Zhang. The limiting equation for the Gross-Pitaevskii equations and S. Terracini's conjectures. J. Funct. Anal., 262(3):1087-1131, 2012.

[16] E. N. Dancer, K. Wang, and Z. Zhang. Uniform Hölder estimate for singularly perturbed parabolic systems of BoseEinstein condensates and competing species. J. Differential Equations, 251(10):2737-2769, 2011.

[17] A. Farina and N. Soave. Monotonicity and 1-dimensional symmetry for solutions of an elliptic system arising in BoseEinstein condensation. Archives Ration. Mech. Anal. 213 (1) (2014), 287-326.

[18] D. Gilbarg and N. S. Trudinger. Elliptic partial differential equations of second order, volume 224 of Grundlehren der Mathematischen Wissenschaften [Fundamental Principles of Mathematical Sciences]. Springer-Verlag, Berlin, second edition, 1983.

[19] N. Matevosyan and A. Petrosyan. Almost monotonicity formulas for elliptic and parabolic operators with variable coefficients. Comm. Pure Appl. Math., 64(2):271-311, 2011.

[20] M. Mimura. Spatial distributions of competiing species, Mathematical Ecology (Trieste, 1982), Lecture Notes in Biomathematics, vol. 54, 1982, 492-501.

[21] B. Noris, H. Tavares, S. Terracini, and G. Verzini. Uniform Hölder bounds for nonlinear Schrödinger systems with strong competition. Comm. Pure Appl. Math., 63(3):267-302, 2010.

[22] A. S. Parkins and D. F. Walls. The physics of trapped dilute-gas Bose-Einstein condensates. Phys. Rep., 303:1-80, 1998.

[23] L. Pitaevskii and S. Stringari. Bose-Einstein condensation. Oxford, 2003.

[24] V. Quitalo. A free boundary problem arising from segregation of populations with high competition. Arch. Ration. Mech. Anal., 210(3):857-908, 2013.

[25] M. Ramos, H. Tavares, and S. Terracini. Existence and regularity of solutions to optimal partition problems involving laplacian eigenvalues. Preprint arXiv:1403.6313

[26] C. Rüegg, N. Cavadini, A. Furrer, H. U. Güdel, K. Krämer, H. Mutka, A. Wildes, K. Habicht, and P. Vorderwisch. Bose-Einstein condensation of the triplet states in the magnetic insulator TlCuCl3. Nature, 423:62-65, 2003.

[27] N. Shigesada, K. Kawasaki, and E. Teramoto. The effects of interference competition on stability, structure and invasion of a multi species system, J. Math. Biol. 21(2)(1984) 97-113.

[28] N. Soave, H. Tavares, and A. Zilio. Quasi-optimal local bounds for strongly competing Schrödinger equations with nontrivial grouping (preprint).

[29] N. Soave and S. Terracini. Liouville theorems and 1-dimensional symmetry for solutions of an elliptic system modelling phase separation. Advances in Math., to appear. Preprint arXiv:1404.7288

[30] N. Soave and A. Zilio, Entire solutions with exponential growth for an elliptic system modeling phase separation. Nonlinearity 27 (2) (2014), 305-342.

[31] H. Tavares and S. Terracini. Regularity of the nodal set of segregated critical configurations under a weak reflection law. Calc. Var. Partial Differential Equations, 45(3-4):273-317, 2012.

[32] S. Terracini, G. Verzini, and A. Zilio. Uniform Hölder bounds for strongly competing systems involving the square root of the laplacian. Preprint arXiv:1211.6087.

[33] S. Terracini, G. Verzini, and A. Zilio. Uniform Hölder regularity with small exponent in competition-fractional diffusion systems. Discrete Contin. Dyn. Syst., 34(6):2669-2691, 2014.

[34] E. Timmermans. Phase separation of Bose-Einstein condensates. Phys. Rev. Lett., 81:5718-5721, 1998.

[35] G. Verzini and A. Zilio. Strong competition versus fractional diffusion: the case of Lotka-Volterra interaction. Comm. Partial Differential Equations, in press doi:10.1080/03605302.2014.890627.

[36] K. Wang. On the De Giorgi type conjecture for an elliptic system modeling phase separation. Comm. Partial Differential Equations, 39(4):696-739, 2014.

[37] K. Wang and Z. Zhang. Some new results in competing systems with many species. Ann. Inst. H. Poincaré Anal. Non Linéaire, 27(2):739-761, 2010.

[38] J. Wei and T. Weth. Asymptotic behaviour of solutions of planar elliptic systems with strong competition. Nonlinearity, 21(2):305-317, 2008. 